\title{
Strong and radiative decays of the low-lying $D$-wave singly heavy baryons
}

\author{
Ya-Xiong Yao, ${ }^{1}$ Kai-Lei Wang, ${ }^{1}$ and Xian-Hui Zhong ${ }^{1,2, *}$ \\ ${ }^{1}$ Department of Physics, Hunan Normal University, and Key Laboratory of Low-Dimensional Quantum \\ Structures and Quantum Control of Ministry of Education, Changsha 410081, China \\ ${ }^{2}$ Synergetic Innovation Center for Quantum Effects and Applications (SICQEA), Hunan Normal \\ University, Changsha 410081, China
}

(Received 22 March 2018; revised manuscript received 28 August 2018; published 25 October 2018)

The strong and radiative decays of the low-lying $\lambda$-mode $D$-wave $\Lambda_{c(b)}, \Sigma_{c(b)}, \Xi_{c(b)}, \Xi_{c(b)}^{\prime}$, and $\Omega_{c(b)}$ baryons are studied in a constituent quark model. Our calculation shows the following: (i) The missing $\lambda$-mode $D$-wave $\Omega_{c(b)}, \Lambda_{b}$, and $\Xi_{b}$ baryons have a relatively narrow decay width of a few $\mathrm{MeV}$ or a few tens of $\mathrm{MeV}$ and their dominant strong and radiative decay channels can be ideal for searching for their signals in future experiments. (ii) The $\lambda$-mode $1 D$-wave excitations in the $\Sigma_{c(b)}$ and $\Xi_{c(b)}^{\prime}$ families appear to have a relatively broad width of $\sim 50-200 \mathrm{MeV}$. Most of the $1 D$-wave states have large decay rates into the $1 P$-wave heavy baryons via the pionic or kaonic strong decay processes, which should be taken seriously in future observations. (iii) Both $\Lambda_{c}(2860)$ and $\Xi_{c}(3050)$ seem to favor the $J^{P}=3 / 2^{+}$excitation $\left|{ }^{2} D_{\lambda \lambda^{2}}{ }^{+}\right\rangle$of $\overline{\mathbf{3}}_{F}$, while both $\Lambda_{c}(2880)$ and $\Xi_{c}(3080)$ may be assigned as the $J^{P}=5 / 2^{+}$excitation $\left|{ }^{2} D_{\lambda \lambda{ }^{2}}{ }^{+}\right\rangle$of $\overline{\mathbf{3}}_{F}$, The nature of $\Xi_{c}(3050)$ and $\Xi_{c}(3080)$ could be tested by the radiative transitions $\Xi_{c}(3055)^{0} \rightarrow \Xi_{c}(2790)^{0} \gamma$ and $\Xi_{c}(3080)^{0} \rightarrow \Xi_{c}(2815)^{0} \gamma$, respectively.

DOI: 10.1103/PhysRevD.98.076015

\section{INTRODUCTION}

The LHC facility provides good opportunities for us to discover some of the missing heavy baryons. Recently, five extremely narrow $\Omega_{c}$ states, $\Omega_{c}(3000), \Omega_{c}(3050)$, $\Omega_{c}(3066), \Omega_{c}(3090)$, and $\Omega_{c}(3119)$, were observed in the $\Xi_{c}^{+} K^{-}$channel by the LHCb Collaboration [1]. Most of them may be interpreted as the $P$-wave excited states of $\Omega_{c}$ [2-9]. Lately, the LHCb Collaboration observed a new structure $\Xi_{b}(6227)^{-}$in both the $\Lambda_{b}^{0} K^{-}$and $\Xi_{b}^{0} \pi^{-}$invariant mass spectra [10]. The mass of this structure and the observed decay modes are consistent with expectations of a $P$-wave excited state in the $\Xi_{b}^{\prime}$ family [11-23]. Besides the missing $P$-wave heavy baryons, some low-lying $D$-wave singly heavy baryons should be also observed at the LHC in forthcoming experiments. Furthermore, the Belle II experiments will also offer the possibility of studying excited heavy baryons. Thus, the theoretical studies of the lowlying $P$ - and $D$-wave singly heavy baryons will provide very useful references for searching for them in future experiments. Considering that the decay properties of

\footnotetext{
*2hongxh@hunnu.edu.cn
}

Published by the American Physical Society under the terms of the Creative Commons Attribution 4.0 International license. Further distribution of this work must maintain attribution to the author(s) and the published article's title, journal citation, and DOI. Funded by SCOAP. heavy baryons should be sensitive to its inner structure, one may better understand the nature of the heavy baryons by studying their decays. In our recent work [11], we systematically studied the strong and radiative decay properties of the $P$-wave singly heavy baryons. As a continuation of Ref. [11], we study the strong and radiative decays of the low-lying $D$-wave singly heavy baryons in the present work.

In the heavy baryon resonances listed in the Review of Particle Physics (RPP) [24], there are several good $D$-wave candidates, such as $\Lambda_{c}(2880)^{+}, \Xi_{c}(3055)^{0,+}, \Xi_{c}(3080)^{0,+}$, and $\Xi_{c}(3123)^{+}$. Recently, a new $D$-wave candidate in the $\Lambda_{c}$ family, i.e., $\Lambda_{c}(2860)^{+}$, was observed in the $D^{0} p$ channel by the LHCb Collaboration [25]. However, no candidates of the $D$-wave bottom baryons have been found in experiments. To look for the missing $D$-wave singly heavy baryons, and to identify these possible $D$-wave heavy baryons observed in experiments, many theoretical studies were carried out with various phenomenological methods. For example, the mass spectra were calculated in various quark models [18-23,26-33], the Faddeev method [34], the lattice QCD [35,36], the QCD sum rules [37-39], and so on. Furthermore, the strong decay properties of the low-lying $D$-wave charmed baryons were also studied within some methods, such as the ${ }^{3} P_{0}$ model $[20,33,40-$ 43], the heavy hadron chiral perturbation theory [44-46], the chiral quark model (ChQM) [47-49], and so on. It should be pointed out that there are few discussions of the 
radiative decays of the $D$-wave charmed and bottom baryons and the strong decays of the $D$-wave bottom baryons, although there are many discussions about the radiative decays [50-68] and strong decays [7-9,69-88] for the low-lying $S$ - and/or $P$-wave singly heavy baryons. More details about the status for the studies of the heavy baryons can be found in Refs. [88-91]. A a whole, it is necessary to carry out a systematical study of the strong and radiative decays for the $D$-wave singly charmed and bottom baryons.

In this work, we apply a nonrelativistic constituent quark model to study the strong decays with emission of one light pseudoscalar meson and the radiative decays with emission of one photon for the low-lying $D$-wave singly heavy baryons. By an analysis of the decay properties for the $D$-wave states, we will suggest ideal decay channels to observe missing states in future experiments. For a simplicity, the harmonic oscillator wave functions of the heavy baryons are adopted in our calculations. To deal with the strong decays of a hadron, an effective chiral Lagrangian at the tree level [92] is introduced. In this interaction, the emitted light pseudoscalar mesons are treated as Goldstone bosons, which only couple with the light constituent quarks. Since the quark-meson coupling is invariant under the chiral transformation, some of the low-energy properties of QCD are retained [92-94]. This method (i.e., ChQM) has been successfully applied to study the strong decays of heavy-light mesons and charmed and strange baryons $[2,47-49,95-100]$. The chiral quark model used in this work is different from the often-used ${ }^{3} P_{0}$ model [101-103]; the differences between them have been pointed out in Ref. [11]. Meanwhile, to treat the radiative decay of a hadron, we apply an effective quark-photon electromagnetic (EM) coupling at the tree level. The higher EM multipole contributions are included by a multipole expansion of the EM interactions. This approach has been successfully applied to deal with the radiative decays of $c \bar{c}$ and $b \bar{b}$ systems [104,105], and recently it has been extended to study the radiative transitions of heavy baryons $[2,99,106]$.

The paper is organized as follows. Section II is our framework, in which we give a brief review of the quark model classification of the singly heavy baryons and the quark model description of the strong and radiative decays. Then, the numerical results for the heavy baryons belonging to $\overline{\mathbf{3}}_{F}$ and $\mathbf{6}_{F}$ are presented and discussed in Secs. III and IV, respectively. Finally, a summary is given in Sec. V.

\section{FRAMEWORK}

\section{A. Spectra}

The heavy baryon containing a heavy quark violates the SU(4) symmetry. However, the SU(3) symmetry between the other two light quarks $(u, d$, or $s)$ is approximately kept. The heavy baryons containing a single heavy quark belong to two different $\mathrm{SU}(3)$ flavor representations: the symmetric sextet $\mathbf{6}_{F}$ and antisymmetric antitriplet $\overline{\mathbf{3}}_{F}$ [11]. In the singly charmed (bottom) baryons, there are two families, $\Lambda_{c}$ and $\Xi_{c}\left(\Lambda_{b}\right.$ and $\left.\Xi_{b}\right)$ belonging to $\overline{\mathbf{3}}_{F}$, while there are three families, $\Sigma_{c}, \Xi_{c}^{\prime}$, and $\Omega_{c}\left(\Sigma_{b}, \Xi_{b}^{\prime}\right.$, and $\left.\Omega_{b}\right)$, belonging to $\mathbf{6}_{F}[11]$.

The spatial wave function of a heavy baryon is adopted the harmonic oscillator form in the constituent quark model [47]. For a $q_{1} q_{2} Q$ basis state, it contains two light quarks $q_{1}$ and $q_{2}$ with equal mass $m$ and a heavy quark $Q$ with mass $m^{\prime}$. The basis states are generated by the oscillator Hamiltonian

$$
\mathcal{H}=\frac{P_{c m}^{2}}{2 M}+\frac{1}{2 m_{\rho}} \mathbf{p}_{\rho}^{2}+\frac{1}{2 m_{\lambda}} \mathbf{p}_{\lambda}^{2}+\frac{3}{2} K\left(\rho^{2}+\lambda^{2}\right)
$$

The constituent quarks are confined in an oscillator potential with the potential parameter $K$ independent of the flavor quantum number. The Jacobi coordinates $\rho$ and $\lambda$ and c.m. coordinate $\mathbf{R}_{\text {c.m. }}$. can be related to the coordinate $\mathbf{r}_{j}$ of the $j$ th quark. The momenta $\mathbf{p}_{\rho}, \mathbf{p}_{\lambda}$, and $\mathbf{P}_{\text {c.m. }}$ are defined by $\mathbf{p}_{\rho}=m_{\rho} \dot{\boldsymbol{\rho}}, \quad \mathbf{p}_{\lambda}=m_{\lambda} \dot{\lambda}, \quad \mathbf{P}_{\text {c.m. }}=M \dot{\mathbf{R}}_{\text {c.m. }}$, with $M=2 m+m^{\prime}$, $m_{\rho}=m$, and $m_{\lambda}=\frac{3 m m^{\prime}}{2 m+m^{\prime}}$. The wave function of an oscillator is give by

$$
\psi_{l_{\sigma} m}^{n_{\sigma}}(\sigma)=R_{n_{\sigma} l_{\sigma}}(\sigma) Y_{l_{\sigma} m}(\sigma),
$$

TABLE I. Mass spectra of the singly heavy baryons of $\overline{\mathbf{3}}_{F}$ up to $D$ wave from various quark models $[21,22,27,33]$ compared with

\begin{tabular}{|c|c|c|c|c|c|c|c|}
\hline \multirow[b]{2}{*}{ State } & \multicolumn{4}{|c|}{$\Lambda_{c}$} & \multicolumn{3}{|c|}{$\Lambda_{b}$} \\
\hline & $\begin{array}{c}\mathrm{RQM} \\
{[21]}\end{array}$ & $\begin{array}{c}\text { NQM } \\
{[27]}\end{array}$ & $\begin{array}{c}\mathrm{NQM} \\
{[33]}\end{array}$ & $\begin{array}{c}\text { PDG } \\
\text { [24] }\end{array}$ & $\begin{array}{c}\text { RQM } \\
{[21]}\end{array}$ & $\begin{array}{c}\text { NQM } \\
{[27]}\end{array}$ & $\begin{array}{c}\text { PDG } \\
{[24]}\end{array}$ \\
\hline $1^{2} S_{2}^{1+}$ & 2286 & 2285 & 2286 & 2286 & 5620 & 5618 & 5620 \\
\hline $1^{2} P_{\lambda 2^{2}}{ }^{-}$ & 2598 & 2628 & 2614 & 2592 & 5930 & 5938 & 5912 \\
\hline $1^{2} P_{\lambda{ }^{3}}^{\frac{3}{2}}-$ & 2627 & 2630 & 2639 & 2628 & 5942 & 5939 & 5920 \\
\hline $1^{2} D_{\lambda \lambda^{2}}{ }^{+}$ & 2874 & 2920 & 2843 & $2860 ?$ & 6190 & 6211 & $?$ \\
\hline \multirow[t]{3}{*}{$1^{2} D_{\lambda \lambda \lambda^{\frac{5}{2}}}$} & 2880 & 2922 & 2851 & $2880 ?$ & 6196 & 6212 & $?$ \\
\hline & \multicolumn{4}{|c|}{$\Xi_{c}$} & \multicolumn{3}{|c|}{$\Xi_{b}$} \\
\hline & $\begin{array}{c}\text { RQM } \\
{[21]}\end{array}$ & $\begin{array}{c}\mathrm{NQM} \\
{[22]}\end{array}$ & $\begin{array}{c}\mathrm{NQM} \\
{[33]}\end{array}$ & $\begin{array}{c}\text { PDG } \\
{[24]}\end{array}$ & $\begin{array}{c}\text { RQM } \\
{[21]}\end{array}$ & $\begin{array}{c}\mathrm{NQM} \\
{[22]}\end{array}$ & $\begin{array}{c}\text { PDG } \\
{[24]}\end{array}$ \\
\hline $1^{2} S \frac{1}{1}+$ & 2476 & 2466 & 2470 & 2468 & 5803 & 5806 & 5795 \\
\hline $1^{2} P_{\lambda 2^{2}}^{2-}$ & 2792 & 2773 & 2793 & 2792 & 6120 & 6090 & $?$ \\
\hline $1^{2} P_{\lambda^{\frac{3}{2}}}-$ & 2819 & 2783 & 2820 & 2817 & 6130 & 6093 & $?$ \\
\hline $1^{2} D_{\lambda \lambda^{2}}{ }^{+}$ & 3059 & 3012 & 3033 & $3055 ?$ & 6366 & 6311 & $?$ \\
\hline $1^{2} D_{\lambda \lambda \frac{5}{2}}{ }^{+}$ & 3076 & 3004 & 3040 & $3080 ?$ & 6373 & 6300 & $?$ \\
\hline
\end{tabular}
the data from the RPP [24]. 
TABLE II. Mass spectra of the singly heavy baryons of $\mathbf{6}_{F}$ up to $D$ wave from various quark models $[21,22,27,33]$ compared with the data from the RPP [24].

\begin{tabular}{|c|c|c|c|c|c|c|c|}
\hline \multirow[b]{2}{*}{ State } & \multicolumn{4}{|c|}{$\Sigma_{c}$} & \multicolumn{3}{|c|}{$\Sigma_{b}$} \\
\hline & $\begin{array}{c}\text { RQM } \\
\text { [21] }\end{array}$ & $\begin{array}{c}\text { NQM } \\
{[27]}\end{array}$ & $\begin{array}{c}\text { NQM } \\
\text { [33] }\end{array}$ & $\begin{array}{c}\text { PDG } \\
{[24]}\end{array}$ & $\begin{array}{c}\text { RQM } \\
{[21]}\end{array}$ & $\begin{array}{c}\text { NQM } \\
{[27]}\end{array}$ & $\begin{array}{c}\text { PDG } \\
{[24]}\end{array}$ \\
\hline $1^{2} S \frac{1}{2}+$ & 2443 & 2460 & 2456 & 2455 & 5808 & 5823 & 5811 \\
\hline $1^{4} S^{\frac{2}{2}}+$ & 2519 & 2523 & 2515 & 2520 & 5834 & 5845 & 5832 \\
\hline $1^{2} P_{\lambda \frac{1}{2}}^{2}$ & 2713 & 2802 & 2702 & $?$ & 6101 & 6127 & $?$ \\
\hline $1^{2} P_{\lambda \frac{3}{2}-}$ & 2798 & 2807 & 2785 & $?$ & 6096 & 6132 & $?$ \\
\hline $1^{4} P_{\lambda} \frac{1}{2}-$ & 2799 & 2826 & 2765 & $?$ & 6095 & 6135 & $?$ \\
\hline $1^{4} P_{\lambda^{\frac{3}{2}}}$ & 2773 & 2837 & 2798 & $?$ & 6087 & 6141 & $?$ \\
\hline $1^{4} P_{\lambda \frac{5}{2}-}$ & 2789 & 2839 & 2790 & $?$ & 6084 & 6144 & $?$ \\
\hline $1^{2} D_{\lambda \lambda^{\frac{3}{2}}}{ }^{+}$ & 3043 & 3065 & 2952 & $?$ & 6326 & 6356 & $?$ \\
\hline $1^{2} D_{\lambda \lambda{ }^{\frac{5}{2}}{ }^{+}}$ & 3038 & 3099 & 2942 & $?$ & 6284 & 6397 & $?$ \\
\hline $1^{4} D_{\lambda \lambda^{\frac{1}{2}}}{ }^{+}$ & 3041 & 3103 & 2949 & $?$ & 6311 & 6395 & $?$ \\
\hline $1^{4} D_{\lambda \lambda^{\frac{3}{2}}}{ }^{+}$ & 3040 & 3094 & 2964 & $?$ & 6285 & 6393 & $?$ \\
\hline $1^{4} D_{\lambda \lambda}{ }^{\frac{5}{2}}$ & 3023 & 3114 & 2962 & $?$ & 6270 & 6402 & $?$ \\
\hline $1^{4} D_{\lambda \lambda \lambda^{2}}{ }^{+}$ & 3013 & $\ldots$ & 2943 & $?$ & 6260 & $\ldots$ & $?$ \\
\hline
\end{tabular}

\begin{tabular}{|c|c|c|c|c|c|c|c|}
\hline \multirow[b]{2}{*}{ State } & \multicolumn{4}{|c|}{$\Xi_{c}^{\prime}$} & \multicolumn{3}{|c|}{$\Xi_{b}^{\prime}$} \\
\hline & $\begin{array}{c}\text { RQM } \\
{[21]}\end{array}$ & $\begin{array}{c}\text { NQM } \\
{[22]}\end{array}$ & $\begin{array}{c}\text { NQM } \\
{[33]}\end{array}$ & $\begin{array}{c}\text { PDG } \\
\text { [24] }\end{array}$ & $\begin{array}{c}\text { RQM } \\
{[21]}\end{array}$ & $\begin{array}{c}\text { NQM } \\
{[22]}\end{array}$ & $\begin{array}{c}\text { PDG } \\
{[24]}\end{array}$ \\
\hline $1^{2} S \frac{1}{2}+$ & 2579 & 2592 & 2579 & 2575 & 5936 & 5958 & 5935 \\
\hline $1^{4} S_{\frac{3}{2}}^{+}+$ & 2649 & 2650 & 2649 & 2645 & 5963 & 5982 & 5955 \\
\hline $1^{2} P_{\lambda \frac{1}{2}-}$ & 2936 & 2859 & 2839 & $?$ & 6233 & 6192 & $?$ \\
\hline & 2935 & 2871 & 2921 & $?$ & 6234 & 6194 & $?$ \\
\hline $1^{4} P_{\lambda} \frac{1}{2}-$ & 2854 & $\ldots$ & 2900 & $?$ & 6227 & $\ldots$ & $?$ \\
\hline $1^{4} P_{\lambda} \lambda^{\frac{3}{2}}$ & 2912 & $\ldots$ & 2932 & $?$ & 6224 & $\ldots$ & $?$ \\
\hline $1^{4} P_{\lambda \frac{5}{2}}$ & 2929 & 2905 & 2927 & $?$ & 6226 & 6204 & $?$ \\
\hline $1^{2} D_{\lambda \lambda^{2}}{ }^{+}$ & 3167 & $\ldots$ & 3089 & $?$ & 6459 & $\ldots$ & $?$ \\
\hline $1^{2} D_{\lambda \lambda \frac{5}{2}^{+}}$ & 3166 & $\cdots$ & 3091 & $?$ & 6432 & 6402 & $?$ \\
\hline $1^{4} D_{\lambda \lambda^{2}}{ }^{+}$ & 3163 & $\ldots$ & 3075 & $?$ & 6447 & $\ldots$ & $?$ \\
\hline $1^{4} D_{\lambda \lambda^{\frac{3}{2}}+}$ & 3160 & $\ldots$ & 3081 & $?$ & 6431 & $\ldots$ & $?$ \\
\hline $1^{4} D_{\lambda \lambda^{\frac{5}{2}}{ }^{+}}$ & 3153 & 3080 & 3077 & $?$ & 6420 & $\ldots$ & $?$ \\
\hline $1^{4} D_{\lambda \lambda \frac{7}{2}^{+}}$ & 3147 & 3094 & 3078 & $?$ & 6414 & 6405 & $?$ \\
\hline
\end{tabular}

\begin{tabular}{|c|c|c|c|c|c|c|c|}
\hline \multirow[b]{2}{*}{ State } & \multicolumn{4}{|c|}{$\Omega_{c}$} & \multicolumn{3}{|c|}{$\Omega_{b}$} \\
\hline & $\begin{array}{c}\text { RQM } \\
{[21]}\end{array}$ & $\begin{array}{c}\text { NQM } \\
{[27]}\end{array}$ & $\begin{array}{c}\mathrm{NQM} \\
{[22]}\end{array}$ & $\begin{array}{c}\text { PDG } \\
{[24]}\end{array}$ & $\begin{array}{c}\text { RQM } \\
{[21]}\end{array}$ & $\begin{array}{c}\text { NQM } \\
{[27]}\end{array}$ & $\begin{array}{c}\text { PDG } \\
{[24]}\end{array}$ \\
\hline $1^{2} S \frac{1}{2}^{+}$ & 2698 & 2731 & 2718 & 2695 & 6064 & 6076 & 6046 \\
\hline $1^{4} S^{\frac{3}{2}}+$ & 2768 & 2779 & 2776 & 2770 & 6088 & 6094 & $?$ \\
\hline $1^{2} P_{\lambda}^{2} \frac{1-}{2}$ & 2966 & 3030 & 2977 & $?$ & 6330 & 6333 & $?$ \\
\hline $1^{2} P_{\lambda \frac{3}{2}-}$ & 3029 & 3033 & 2986 & $?$ & 6331 & 6336 & $?$ \\
\hline $1^{4} P_{\lambda \frac{1}{2}-}$ & 3055 & 3048 & 2990 & $?$ & 6339 & 6340 & $?$ \\
\hline $1^{4} P_{\lambda{ }^{\frac{3}{2}}-}$ & 3054 & 3056 & 2994 & $?$ & 6340 & 6344 & $?$ \\
\hline $1^{4} P_{\lambda \frac{5}{2}}^{-}$ & 3051 & 3057 & 3014 & $?$ & 6334 & 6345 & $?$ \\
\hline $1^{2} D_{\lambda \lambda^{3}}{ }^{+}$ & 3282 & 3257 & 3262 & $?$ & 6530 & 6528 & $?$ \\
\hline $1^{2} D_{\lambda \lambda^{2}}{ }^{+}$ & 3286 & 3288 & 3273 & $?$ & 6520 & 6561 & $?$ \\
\hline $1^{4} D_{\lambda \lambda}{ }^{\frac{1}{2}}{ }^{+}$ & 3287 & 3292 & 3275 & $?$ & 6540 & 6561 & $?$ \\
\hline $1^{4} D_{\lambda \lambda^{\frac{3}{2}}}{ }^{+}$ & 3298 & 3285 & 3280 & $?$ & 6549 & 6559 & $?$ \\
\hline $1^{4} D_{\lambda \lambda} \lambda^{\frac{5}{2}}$ & 3297 & 3299 & $\ldots$ & $?$ & 6529 & 6566 & $?$ \\
\hline $1^{4} D_{\lambda \lambda \overline{2}^{+}}{ }^{+}$ & 3283 & $\ldots$ & 3327 & $?$ & 6517 & $\ldots$ & $?$ \\
\hline
\end{tabular}

where $\sigma \equiv \rho, \lambda$. In the wave functions, there are two oscillator parameters, i.e., the potential strengths $\alpha_{\rho}$ and $\alpha_{\lambda}$. The parameters $\alpha_{\rho}$ and $\alpha_{\lambda}$ satisfy the following relation [47]:

$$
\alpha_{\lambda}^{2}=\sqrt{\frac{3 m^{\prime}}{2 m+m^{\prime}}} \alpha_{\rho}^{2} .
$$

The details of the classifications of the heavy baryons in the constituent quark model can be found in Refs. [47]. Considering that the $\lambda$-mode excitations of singly heavy baryons should be easily formed than the $\rho$-mode excitations [27], thus in the present work, we only study the $\lambda$-mode excitations. The mass spectra of the single heavy baryons up to the $1 D$-wave excitations predicted within various quark models are summarized in Tables I and II.

\section{B. Decays}

In this work, strong decays of the $D$-wave singly heavy baryons with emission of one light pseudoscalar meson are studied within ChQM [92]. This model has been successfully applied to study the strong decays of heavy-light mesons and charmed and strange baryons [2,47-49,95100]. In this model, the light pseudoscalar mesons, i.e., $\pi$, $K$, and $\eta$, are treated as fundamental states, which only couple with the light constituent quarks of a hadron via the simple chiral Lagrangian [92]

$$
H_{m}=\sum_{j} \frac{1}{f_{m}} \bar{\psi}_{j} \gamma_{\mu}^{j} \gamma_{5}^{j} \psi_{j} \partial^{\mu} \phi_{m},
$$

where $\psi_{j}$ represents the $j$ th quark field in the hadron, $\phi_{m}$ is the pseudoscalar meson field, and $f_{m}$ is the pseudoscalar meson decay constant.

Meanwhile, to treat the radiative decay of a hadron, we apply the constituent quark model, which has been successfully applied to study the radiative decays of $c \bar{c}$ and $b \bar{b}$ systems $[104,105]$. In this model, the quark-photon EM coupling at the tree level is adopted as

$$
H_{e}=-\sum_{j} e_{j} \bar{\psi}_{j} \gamma_{\mu}^{j} A^{\mu}\left(\mathbf{k}, \mathbf{r}_{j}\right) \psi_{j}
$$

where $A^{\mu}$ represents the photon field with 3-momenta $\mathbf{k} . e_{j}$ and $\mathbf{r}_{j}$ stand for the charge and coordinate of the constituent quark $\psi_{j}$, respectively.

To match the nonrelativistic harmonic oscillator wave functions, in the calculations, one should adopt the nonrelativistic forms for the quark-pseudoscalar and quarkphoton EM couplings listed in Eqs. (4) and (5), which have been given in the previous works $[2,47,48,93-100,104-112]$.

For a strong decay process, the partial decay width can be calculated with [47] 
TABLE III. Quark model parameters adopted in present work.

\begin{tabular}{lcccccccc}
\hline \hline Parameter & $m_{u(d)}$ & $m_{s}$ & $m_{c}$ & $m_{b}$ & $\alpha_{\rho}\left(\right.$ for $\Lambda_{c(b)}$ and $\left.\Sigma_{c(b)}\right)$ & $\alpha_{\rho}\left(\right.$ for $\left.\Xi_{c(b)}^{(\prime)}\right)$ & $\alpha_{\rho}\left(\right.$ for $\left.\Omega_{c(b)}\right)$ & $f_{\pi}$ \\
Value $(\mathrm{MeV})$ & 330 & 450 & 1480 & 5000 & 400 & 420 & 440 & 132 \\
\hline \hline
\end{tabular}

$$
\Gamma_{m}=\left(\frac{\delta}{f_{m}}\right)^{2} \frac{\left(E_{f}+M_{f}\right)|\mathbf{q}|}{4 \pi M_{i}\left(2 J_{i}+1\right)} \sum_{J_{f z}, J_{i z}}\left|\mathcal{M}_{J_{f z}, J_{i z}}\right|^{2}
$$

while for a radiative decay process, the partial decay width can be calculated with $[104,105]$

$$
\Gamma_{\gamma}=\frac{|\mathbf{k}|^{2}}{\pi} \frac{2}{2 J_{i}+1} \frac{M_{f}}{M_{i}} \sum_{J_{f_{z}}, J_{i z}}\left|\mathcal{A}_{J_{f z}, J_{i z}}\right|^{2}
$$

where $\mathcal{M}_{J_{f z}, J_{i z}}$ and $\mathcal{A}_{J_{f z}, J_{i z}}$ correspond to the strong and radiative transition amplitudes, respectively. The quantum numbers $J_{i z}$ and $J_{f z}$ stand for the third components of the total angular momenta of the initial and final heavy baryons, respectively. $E_{f}$ and $M_{f}$ are the energy and mass of the final heavy baryon, and $M_{i}$ is the mass of the initial heavy baryon. $\delta$ as a global parameter accounts for the strength of the quark-meson couplings. It has been determined in our previous study of the strong decays of the charmed baryons and heavy-light mesons [47,96]. Here, we fix its value the same as that in Refs. $[47,96]$, i.e., $\delta=0.557$.

In the calculation, we adopt the same quark model parameter set as that in Ref. [11], which has been collected in Table III. The masses of the well-established hadrons used in the calculations are adopted from the RPP [24].

\section{RESULTS FOR SINGLY HEAVY BARYONS OF $\overline{3}_{F}$}

\section{A. $\Lambda_{c}$ states}

In the $\Lambda_{c}$ family, there are two $\lambda$-mode $1 D$-wave excitations $\left|\Lambda_{c}{ }^{2} D_{\lambda \lambda^{2}}{ }^{+}\right\rangle$and $\left|\Lambda_{c}{ }^{2} D_{\lambda \lambda^{\frac{5}{2}}}{ }^{+}\right\rangle$according to the quark model classification. The masses for the $\lambda$-mode $1 D$ wave $\Lambda_{c}$ excitations are predicted to be $\sim 2.9 \mathrm{GeV}$ in various models (see Table I). The resonances $\Lambda_{c}(2860)$ with $J^{P}=3 / 2^{+}$and $\Lambda_{c}(2880)$ with $J^{P}=5 / 2^{+}$listed in RPP [24] most likely correspond to the two $\lambda$-mode $1 D$-wave $\Lambda_{c}$ excitations $\left|\Lambda_{c}{ }^{2} D_{\lambda \lambda^{2}}{ }^{+}\right\rangle$and $\left|\Lambda_{c}{ }^{2} D_{\lambda \lambda^{2}}{ }^{+}\right\rangle$, respectively.

\section{1. $J^{P}=5 / 2^{+}$state and $\Lambda_{c}(2880)$}

The $\Lambda_{c}(2880)$ state was first observed in $\Lambda_{c}^{+} \pi^{+} \pi^{-}$by CLEO [113]. It was confirmed in $\Sigma_{c} \pi$ and $\Sigma_{c}(2520) \pi$ channels by Belle [114] and in the $D^{0} p$ channel by BABAR [115] and LHCb [25]. It has a narrow decay width of $\Gamma \simeq 5.6 \mathrm{MeV}$ [24]. The spin-parity numbers were determined to be $J^{P}=5 / 2^{+}$by Belle [114] and were confirmed by $\mathrm{LHCb}$ [25] recently.

The $\Lambda_{c}(2880)$ state may be classified as the $1 D$-wave charmed baryons $[23,30,34,40,44]$. If $\Lambda_{c}(2880)$ is a conventional $\lambda$-mode $1 D$-wave excitation, it should be assigned to $\left|\Lambda_{c}^{2} D_{\lambda \lambda^{\frac{5}{2}}}{ }^{+}\right\rangle$. With this assignment, the width of $\Lambda_{c}(2880)$ can be reasonably understood by ChQM [47]. It is found that the main decay channel of $\Lambda_{c}(2880)$ should be $\Sigma_{c}(2520) \pi$ (see Table IV). The partial width ratio, $\mathcal{R}=\frac{\Gamma\left[\Sigma_{c}(2520) \pi\right]}{\Gamma\left[\Sigma_{c}(2455) \pi\right]} \simeq 3.3$, predicted by us is too large to compare with the measured value $\mathcal{R} \simeq 0.225$ at Belle [114]. The recent ${ }^{3} P_{0}$ analysis of the strong decays of $\Lambda_{c}(2880)$ in Ref. [41] is consistent with our predictions.

It should be mentioned that the measured ratio $\mathcal{R}=$ $\frac{\Gamma\left[\Sigma_{c}(2520) \pi\right]}{\Gamma\left[\Sigma_{c}(2455) \pi\right]} \simeq 0.225$ of $\Lambda_{c}(2880)$ may be strongly affected by its nearby state $\Lambda_{c}(2860) \frac{3}{2}^{+}$newly observed in the $D^{0} p$ channel at $\mathrm{LHCb}[25]$. Thus, the measured ratio from Belle [114] should not be a genuine ratio for $\Lambda_{c}(2880)$. This situation is very similar to that of $D_{s J}(2860)$ before two largely overlapping states $D_{s 1}(2860)$ and $D_{s 3}(2860)$ were found by LHCb [116]. Considering $D_{s J}(2860)$ as the $J^{P}=3^{-}$state $1^{3} D_{3}$, the measured partial width ratio $\mathcal{R}=$ $\frac{\Gamma\left[D^{*} K\right]}{\Gamma[D K]} \simeq 1.1$ cannot be explained by ChQM $[95,96]$ and

TABLE IV. Partial widths of strong decays for the $\lambda$-mode $D$ wave $\Lambda_{c}$ and $\Lambda_{b}$ baryons. The masses of the $D$-wave $\Lambda_{c}\left(\Lambda_{b}\right)$ states $\left|{ }^{2} D_{\lambda \lambda}{ }^{\frac{3}{2}}\right\rangle$ and $\left|{ }^{2} D_{\lambda \lambda^{2}}{ }^{+}\right\rangle$are taken as 2856 and 2881 (6190 and 6196) $\mathrm{MeV}$, respectively. The superscript (subscript) stands for the uncertainty of a prediction with a $+10 \%(-10 \%)$

\begin{tabular}{|c|c|c|}
\hline & $\left|\Lambda_{c}^{2} D_{\lambda \lambda^{\frac{3}{2}}}{ }^{\prime}\right\rangle(2856)$ & $\left|\Lambda_{c}^{2} D_{\lambda \lambda^{\frac{5}{2}}}{ }^{+}\right\rangle(2881)$ \\
\hline Decay mode & $\Gamma_{i}(\mathrm{MeV})$ & $\Gamma_{i}(\mathrm{MeV})$ \\
\hline$\Sigma_{c} \pi$ & $4.57_{-1.20}^{+1.09}$ & $1.33_{+0.50}^{-0.35}$ \\
\hline$\Sigma_{c}^{*} \pi$ & $0.95_{+0.09}^{-0.03}$ & $4.38_{-0.74}^{+0.50}$ \\
\hline \multirow[t]{2}{*}{ Sum } & $5.52_{-1.11}^{+1.06}$ & $5.71_{-0.24}^{+0.32}$ \\
\hline & $\left|\Lambda_{b}^{2} D_{\lambda \lambda^{\frac{3}{2}}}{ }^{+}\right\rangle(6190)$ & $\left|\Lambda_{b}^{2} D_{\lambda \lambda^{\frac{5}{2}}}{ }^{+}\right\rangle(6196)$ \\
\hline Decay mode & $\Gamma_{i}(\mathrm{MeV})$ & $\Gamma_{i}(\mathrm{MeV})$ \\
\hline$\Sigma_{b} \pi$ & $6.32_{-1.91}^{+1.78}$ & $1.83_{+0.67}^{-0.47}$ \\
\hline$\Sigma_{b}^{*} \pi$ & $2.76_{+0.41}^{-0.22}$ & $7.42_{-1.43}^{+1.38}$ \\
\hline Sum & $9.08_{-1.50}^{+1.56}$ & $9.25_{-0.76}^{+0.91}$ \\
\hline
\end{tabular}
uncertainty of the oscillator parameter $\alpha_{\rho}$. 
many other approaches [117-119]. Then, people proposed an alternative solution that there might exist two largely overlapping resonances at about $2.86 \mathrm{GeV}[95,120]$, which was confirmed by LHCb recently [116].

To better understand the properties of $\Lambda_{c}(2880)$, considering it as the $1 D$-wave state $\left|\Lambda_{c}{ }^{2} D_{\lambda \lambda^{2}}{ }^{+}\right\rangle$, we further study its radiative decays into the $1 P$-wave charmed baryon states. Our results are listed in Table V. It is found that most of the partial radiative widths of $\Lambda_{c}(2880)$ into the $1 P$-wave states are $\mathcal{O}(100) \mathrm{eV}$. Combining these partial widths with the total decay width of $\Lambda_{c}(2880)$, we find the branching fractions for the main radiative decay channels are $\mathcal{O}\left(10^{-5}\right)$. The small decay rates indicate that the radiative decays of $\Lambda_{c}(2880)$ into the $1 P$-wave states might be hard to be observed in experiments.

\section{2. $J^{P}=3 / 2^{+}$state and $\Lambda_{c}(\mathbf{2 8 6 0})$}

Recently, besides the confirmation of $\Lambda_{c}(2880)$ in the $D^{0} p$ channel, the LHCb Collaboration observed a new charmed baryon state, $\Lambda_{c}(2860)$, with a broad width of $\Gamma \simeq 67.6_{-8.1}^{+10.1} \mathrm{MeV}$ in the same channel [25]. The determined spin-parity quantum numbers are $J^{P}=3 / 2^{+}$[25].

TABLE V. Partial widths of radiative decays for the $\lambda$-mode $D$ wave $\Lambda_{c}$ and $\Lambda_{b}$ baryons. The masses of the $D$-wave $\Lambda_{c}\left(\Lambda_{b}\right)$ states $\left|{ }^{2} D_{\lambda \lambda \lambda^{\frac{3}{2}}}{ }^{+}\right\rangle$and $\left|{ }^{2} D_{\lambda \lambda \frac{5}{2}}{ }^{+}\right\rangle$are taken as 2856 and 2881 (6190 and 6196) $\mathrm{MeV}$, respectively. $M_{f}$ stands for the masses of $P$-wave heavy baryons $(\mathrm{MeV})$ in the final states, which are adopted from the RPP [24] and Ref. [21]. The superscript (subscript) stands for the uncertainty of a prediction with a $+10 \%(-10 \%)$ uncertainty of the oscillator parameter $\alpha_{\rho}$.

\begin{tabular}{|c|c|c|c|}
\hline & & $\left|\Lambda_{c}^{2} D_{\lambda \lambda 2^{\frac{3}{2}}}\right\rangle(2856)$ & $\left|\Lambda_{c}^{2} D_{\lambda \lambda}{ }^{\frac{5}{2}}\right\rangle(2881)$ \\
\hline Decay mode & $M_{f}$ & $\Gamma_{i}(\mathrm{keV})$ & $\Gamma_{i}(\mathrm{keV})$ \\
\hline$\left|\Lambda_{c}^{+2} P_{\lambda \frac{1}{2}-}\right\rangle \gamma$ & 2592 & 0.01 & $0.13_{+0.03}^{-0.03}$ \\
\hline$\left|\Lambda_{c}^{+2} P_{\lambda^{\frac{3}{2}}}\right\rangle^{\prime}$ & 2628 & 0.07 & $0.26_{+0.03}^{+0.03}$ \\
\hline$\left|\Sigma_{c}^{+2} P_{\lambda \frac{1}{2}-}\right\rangle \gamma$ & 2713 & $0.23_{+0.05}^{-0.04}$ & $0.80_{+0.15}^{-0.03}$ \\
\hline$\left|\Sigma_{c}^{+2} P_{\lambda 2^{-}}{ }^{-}\right\rangle \gamma$ & 2798 & 0.01 & 0.05 \\
\hline$\left|\Sigma_{c}^{+4} P_{\lambda} \frac{1}{2}-\right\rangle \gamma$ & 2799 & $<0.01$ & $<0.01$ \\
\hline$\left|\Sigma_{c}^{+4} P_{\lambda \lambda^{3}}-\right\rangle \gamma$ & 2773 & 0.08 & $0.13_{+0.03}^{-0.02}$ \\
\hline$\left.\left|\Sigma_{c}^{+4} P_{\lambda{ }^{2}}\right\rangle^{-}\right\rangle \gamma$ & 2789 & $<0.01$ & $0.19_{+0.04}^{-0.03}$ \\
\hline
\end{tabular}

\begin{tabular}{|c|c|c|c|}
\hline & & $\left|\Lambda_{b}{ }^{2} D_{\lambda \lambda^{2}}{ }^{3+}\right\rangle(6190)$ & $\left|\Lambda_{b}^{2} D_{\lambda \lambda \lambda^{\frac{5}{2}}}{ }^{+}\right\rangle(6196)$ \\
\hline Decay mode & $M_{f}$ & $\Gamma_{i}(\mathrm{keV})$ & $\Gamma_{i}(\mathrm{keV})$ \\
\hline$\left|\Lambda_{b}^{02} P_{\lambda{ }^{2}}{ }^{-}\right\rangle \gamma$ & 5912 & $19.7_{+3.7}^{-3.0}$ & $1.67_{+0.49}^{-0.34}$ \\
\hline$\left|\Lambda_{b}^{02} P_{\lambda{ }^{2}}{ }^{-}\right\rangle \gamma$ & 5920 & $6.26_{+1.45}^{-1.08}$ & $24.1_{+4.7}^{-3.7}$ \\
\hline$\left|\Sigma_{b}^{02} P_{\lambda \frac{1}{2}}^{1-}\right\rangle \gamma$ & 6101 & 0.04 & 0.09 \\
\hline$\left|\Sigma_{b}^{02} P_{\lambda 2^{3}}-\right\rangle \gamma$ & 6096 & $0.17_{+0.04}^{-0.03}$ & $0.18_{+0.03}^{-0.03}$ \\
\hline$\left|\Sigma_{b}^{04} P_{\lambda} \lambda^{\frac{1}{2}}-\right\rangle \gamma$ & 6095 & 0.08 & 0.01 \\
\hline$\left|\Sigma_{b}^{04} P_{\lambda^{2}}{ }^{\frac{3}{2}}\right\rangle \gamma$ & 6087 & $0.34_{+0.07}^{-0.05}$ & $0.21_{+0.04}^{-0.04}$ \\
\hline$\left|\Sigma_{b}^{04} P_{\lambda{ }^{2}}{ }^{-}\right\rangle \gamma$ & 6084 & $0.11_{+0.02}^{-0.02}$ & $0.75_{+0.16}^{-0.12}$ \\
\hline
\end{tabular}

Both the mass and decay modes of $\Lambda_{c}(2860)$ indicate that it might be assigned to the $\lambda$-mode excited $1 D$-wave charmed baryon state $\left|\Lambda_{c}{ }^{2} D_{\lambda \lambda^{2}}{ }^{3}\right\rangle[21,33,41]$. Considering $\Lambda_{c}(2860)$ as the $\left|\Lambda_{c}{ }^{2} D_{\lambda \lambda \frac{3}{2}}{ }^{+}\right\rangle$state, we predict its partial widths into the $\Sigma_{c}(2455) \pi$ and $\Sigma_{c}(2520) \pi$ channels,

$\Gamma\left[\Sigma_{c}(2455) \pi\right] \simeq 4.6 \mathrm{MeV}, \quad \Gamma\left[\Sigma_{c}(2520) \pi\right] \simeq 1.0 \mathrm{MeV}$,

which roughly agree with the predictions in Ref. [41]. Combining these predicted partial widths with the measured width of $\Lambda_{c}(2860)$, we further estimate that the branching fractions of the $\Sigma_{c}(2455) \pi$ and $\Sigma_{c}(2520) \pi$ channels can reach up to $7 \%$ and $2 \%$, respectively. The relatively large branching fractions indicate that $\Lambda_{c}(2860)$ might be observed in the $\Sigma_{c}(2455) \pi$ and $\Sigma_{c}(2520) \pi$ channels as well.

Considering $\Lambda_{c}(2860)$ as the $1 D$-wave state $\left|\Lambda_{c}^{2} D_{\lambda \lambda^{3}}{ }^{+}\right\rangle$, we also study its radiative decays into the $1 P$-wave states. Our results are listed in Table V as well. It is found that the radiative decay rates into the $1 P$-wave states are small. Their partial decay widths are $\mathcal{O}(10) \mathrm{eV}$. Combining these partial widths with the total decay width of $\Lambda_{c}(2860)$, we find the branching fractions, $\mathcal{B}\left[\Lambda_{c}(2860) \rightarrow 1 P \gamma\right]$, are $\mathcal{O}\left(10^{-6}\right)$, which indicates the radiative decays of $\Lambda_{c}(2860)$ into the $1 P$-wave states might be hard to be observed in experiments.

\section{B. $\Lambda_{b}$ states}

In the $\Lambda_{b}$ family, there are two $\lambda$-mode $1 D$-wave excitations $\left|\Lambda_{b}^{2} D_{\lambda \lambda^{2}}{ }^{+}\right\rangle$and $\left|\Lambda_{b}^{2} D_{\lambda \lambda^{2}}{ }^{+}\right\rangle$according to the quark model classification. The masses for the $\lambda$-mode $1 D$-wave $\Lambda_{b}$ excitations are predicted to be $\sim 6.2 \mathrm{GeV}$ in various models (see Table I). In the possible mass region of the $1 D$-wave $\Lambda_{b}$ excitations, we study their strong decay properties, which have been shown in Fig. 1. To be more specific, taking the masses of the $1 D$-wave states as predicted in the relativistic quark-diquark picture [21], we further present the results in Table IV.

$$
\text { 1. } J^{P}=3 / 2^{+} \text {state }
$$

From Fig. 1, it is found that if the mass of $\left|\Lambda_{b}{ }^{2} D_{\lambda \lambda}{ }^{\frac{3}{2}}\right\rangle$ is $\sim 6200 \mathrm{MeV}$ as predicted in theory $[21,27]$ it should be a narrow state with a width of $\Gamma_{\text {total }} \simeq 10 \mathrm{MeV}$. The decays may be saturated by the $\Sigma_{b} \pi$ and $\Sigma_{b}^{*} \pi$ channels, and the partial width ratio between them is predicted to be

$$
\frac{\Gamma\left(\Sigma_{b} \pi\right)}{\Gamma\left(\Sigma_{b}^{*} \pi\right)} \simeq 2 \text {, }
$$

which is less sensitive to the mass of $\left|\Lambda_{b}^{2} D_{\lambda \lambda} \lambda^{\frac{3}{2}}{ }^{+}\right\rangle$. On the other hand, if the mass of $\left|\Lambda_{b}^{2} D_{\lambda \lambda^{\frac{3}{2}}}{ }^{+}\right\rangle$is larger than $6240 \mathrm{MeV}$, more strong decay channels may open. The 


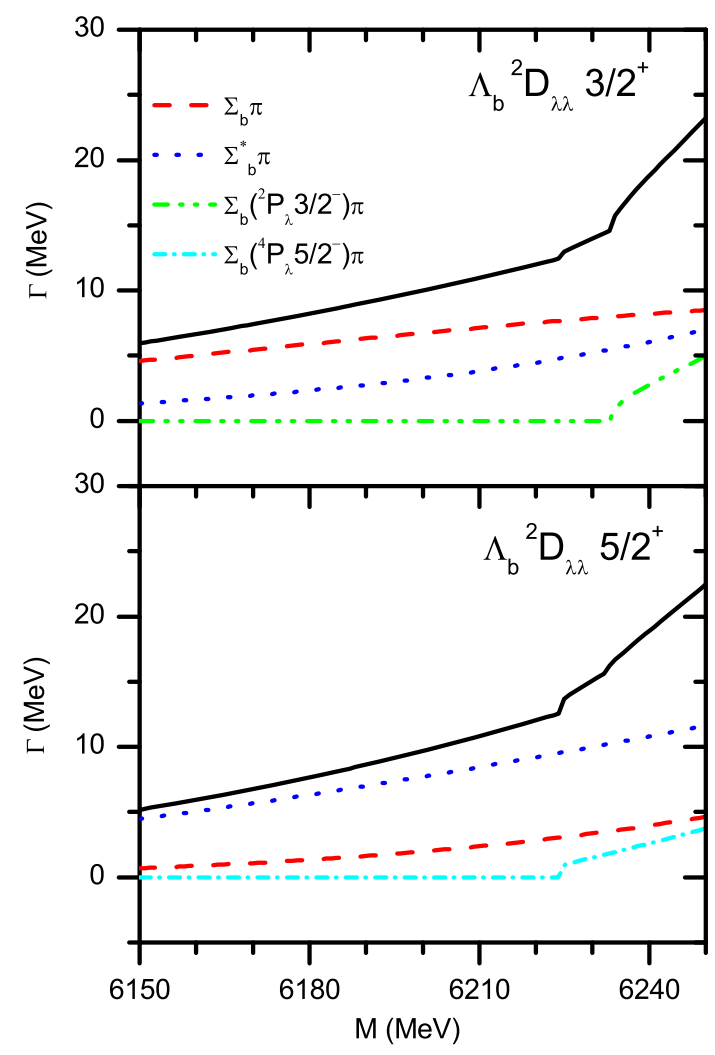

FIG. 1. Strong decay partial widths of the main decay modes for the $\lambda$-mode $1 D$-wave excited $\Lambda_{b}$ states $\left|\Lambda_{b} D_{\lambda \lambda} 3 / 2^{+}\right\rangle$and $\left|\Lambda_{b} D_{\lambda \lambda} 5 / 2^{+}\right\rangle$as functions of their mass. The solid curves stand for the total widths. The masses of the $P$-wave heavy baryons in final states are adopted from the quark model predictions in Ref. [21] (see Table II).

$\left|\Lambda_{b}^{2} D_{\lambda \lambda^{3}}{ }^{3}\right\rangle$ state may have a large decay rate into the $\left|\Sigma_{b}^{2} P_{\lambda}{ }^{\frac{3}{2}}\right\rangle \pi$ channel as well (see Fig. 1). To establish the missing $D$-wave state $\left|\Lambda_{b}^{2} D_{\lambda \lambda \frac{3}{2}}{ }^{+}\right\rangle$, the decay channel $\Sigma_{b} \pi$ might be the ideal channel to be observed in future experiments.

We further estimate the radiative decays of $\left|\Lambda_{b}^{2} D_{\lambda \lambda^{\frac{3}{2}}}{ }^{+}\right\rangle$ into the $1 P$-wave states. Our results are listed in Table V. It is found that $\left|\Lambda_{b}^{2} D_{\lambda \lambda^{3}}{ }^{+}\right\rangle$has a relatively large decay rate into $\Lambda_{b}(5912) \frac{1}{2}-\gamma$, and the partial width of $\Gamma\left[\left|\Lambda_{b}^{2} D_{\lambda \lambda} \frac{3}{2}^{+}\right\rangle \rightarrow\right.$ $\left.\Lambda_{b}(5912) \frac{1}{2}-\gamma\right]$ can reach up to $\sim 20 \mathrm{keV}$. Combining it with our predicted total width, we find the branching fraction of $\mathcal{B}\left[\left|\Lambda_{b}^{2} D_{\lambda \lambda^{\frac{3}{2}}}{ }^{+}\right\rangle \rightarrow \Lambda_{b}(5912) \gamma\right]$ is $\mathcal{O}\left(10^{-3}\right)$, which indicates that $\left|\Lambda_{b}^{2} D_{\lambda \lambda^{3}}{ }^{+}\right\rangle$has the possibility of being observed in the $\Lambda_{b}(5912) \frac{1}{2}-\gamma$ channel.

\section{2. $J^{P}=5 / 2^{+}$state}

If the mass of $\left|\Lambda_{b}^{2} D_{\lambda 2^{2}}{ }^{+}\right\rangle$is less than $6200 \mathrm{MeV}$ as predicted in various quark models [21,27], the decays of $\left|\Lambda_{b}^{2} D_{\lambda \lambda^{2}}{ }^{+}\right\rangle$may be saturated by the $\Sigma_{b} \pi$ and $\Sigma_{b}^{*} \pi$ channels. The $\left|\Lambda_{b}{ }^{2} D_{\lambda \lambda^{2}}{ }^{5}\right\rangle$ state has a narrow width of $\Gamma_{\text {total }} \simeq$ $10 \mathrm{MeV}$, which is comparable with that of $\left|\Lambda_{b}{ }^{2} D_{\lambda \lambda^{\frac{3}{2}}}{ }^{+}\right\rangle$ (see Fig. 1). However, the strong decays of $\left|\Lambda_{b}{ }^{2} D_{\lambda \lambda^{2}}{ }^{+}\right\rangle$are governed by the $\Sigma_{b}^{*} \pi$ channel. The partial width ratio between $\Sigma_{b} \pi$ and $\Sigma_{b}^{*} \pi$ is predicted to be

$$
\frac{\Gamma\left(\Sigma_{b} \pi\right)}{\Gamma\left(\Sigma_{b}^{*} \pi\right)} \simeq 0.25,
$$

which shows few sensibilities to the mass of $\left|\Lambda_{b}{ }^{2} D_{\lambda \lambda^{2}}{ }^{5}\right\rangle$. On the other hand, if the mass of $\left|\Lambda_{b}{ }^{2} D_{\lambda \lambda \frac{5}{2}}{ }^{+}\right\rangle$is larger than $6240 \mathrm{MeV}$, more strong decay channels may open. The $\left|\Sigma_{b}{ }^{4} P{ }^{5}{ }^{-}\right\rangle \pi$ decay mode may play an important role in the decays as well. To establish the missing $D$-wave state $\left|\Lambda_{b}^{2} D_{\lambda \lambda^{2}}{ }^{+}\right\rangle$, the decay channel $\Sigma_{b}^{*} \pi$ should be the ideal channel to be observed in future experiments.

To know more properties of $\left|\Lambda_{b}^{2} D_{\lambda \lambda^{2}}{ }^{+}\right\rangle$, we further estimate its radiative decays into the $1 P$-wave states. Our results are listed in Table V. It is found that the radiative process $\left|\Lambda_{b}{ }^{2} D_{\lambda \lambda \frac{5}{2}}{ }^{+}\right\rangle \rightarrow \Lambda_{b}(5920) \frac{3}{2}{ }^{-} \gamma$ has a relatively large partial width $\sim 24 \mathrm{keV}$. Combining it with our predicted total width, we find the branching fraction of $\mathcal{B}\left[\left|\Lambda_{b}^{2} D_{\lambda \lambda^{2}}{ }^{+}\right\rangle \rightarrow\right.$ $\left.\Lambda_{b}(5920) \frac{3}{2}^{-} \gamma\right]$ is $\mathcal{O}\left(10^{-3}\right)$, which indicates that $\left|\Lambda_{b}{ }^{2} D_{\lambda \lambda^{2}}{ }^{+}\right\rangle$ has the possibility of being observed in the $\Lambda_{b}(5920) \frac{3}{2}^{-} \gamma$ channel.

\section{C. $\boldsymbol{\Xi}_{c}$ states}

In the $\Xi_{c}$ family, there are two $\lambda$-mode $1 D$-wave

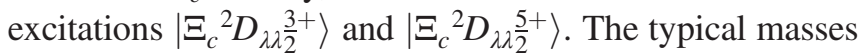
of the $\lambda$-mode $1 D$-wave $\Xi_{c}$ excitations are $\sim 3.05 \mathrm{GeV}$ within various quark model predictions (see Table I). From the point of view of mass, the charmed-strange baryons $\Xi_{c}(3055)^{+}$and $\Xi_{c}(3080)^{+}$observed in the $\Lambda_{c} \bar{K} \pi$ final state by the Belle [121] and BABAR [122] Collaborations are good candidates of the $\lambda$-mode $1 D$-wave states. Recently, a new decay mode $D^{+} \Lambda$ for both $\Xi_{c}(3055)^{+}$and $\Xi_{c}(3080)^{+}$ was observed by the Belle Collaboration [123]. They first reported the following partial width ratios:

$\frac{\Gamma\left[\Xi_{c}(3055)^{+} \rightarrow \Lambda D^{+}\right]}{\Gamma\left[\Xi_{c}(3055)^{+} \rightarrow \Sigma_{c}(2455)^{++} K^{-}\right]}=5.09 \pm 1.01 \pm 0.76$,

$\frac{\Gamma\left[\Xi_{c}(3080)^{+} \rightarrow \Lambda D^{+}\right]}{\Gamma\left[\Xi_{c}(3080)^{+} \rightarrow \Sigma_{c}(2455)^{++} K^{-}\right]}=1.29 \pm 0.30 \pm 0.15$,

and

$\frac{\Gamma\left[\Xi_{c}(3080)^{+} \rightarrow \Sigma_{c}(2520)^{++} K^{-}\right]}{\Gamma\left[\Xi_{c}(3080)^{+} \rightarrow \Sigma_{c}(2455)^{++} K^{-}\right]}=1.07 \pm 0.27 \pm 0.01$.

Furthermore, more accurate widths for both $\Xi_{c}(3055)^{+}$ and $\Xi_{c}(3080)^{+}$were obtained by the Belle Collaboration, 
i.e., $\Gamma_{\Xi_{c}(3055)^{+}}=7.8 \pm 1.1 \pm 1.5$ and $\Gamma_{\Xi_{c}(3080)^{+}}=3.0 \pm 0.7 \pm$ $0.4 \mathrm{MeV}$ [123].

\section{1. $J^{P}=3 / 2^{+}$state and $\Xi_{c}(3055)$}

In Ref. [48], the strong decay properties of the $1 D$-wave states were studied within ChQM. It is found that $\Xi_{c}(3055)$ seems to favor the $J^{P}=3 / 2^{+}$state $\left|\Xi_{c}{ }^{2} D_{\lambda \lambda^{2}}{ }^{+}\right\rangle$, which is consistent with the predictions in Refs. [21,41]. Based on the SU(4) symmetry, we estimated the partial width of $\Gamma\left[\Xi_{c}(3055)^{+} \rightarrow \Lambda D^{+}\right]$, which is too small to compare with the observation at Belle [123]. The serious SU(4) symmetry breaking might lead to our failed description of the decays into the $D^{+} \Lambda$ channel. Assigning $\Xi_{c}(3055)$ as $\left|\Xi_{c}^{2} D_{\lambda \lambda^{3}}{ }^{+}\right\rangle$, it should have relatively large decay rates into $\Xi_{c}^{\prime} \pi$ and $\Sigma_{c}(2455) K$ channels (see Table VI). The predicted partial width ratio between these two channels is

$$
\frac{\Gamma\left[\Xi_{c}^{\prime 0} \pi^{+}\right]}{\Gamma\left[\Sigma_{c}(2455)^{++} K^{-}\right]} \simeq 0.78
$$

TABLE VI. Partial widths of strong decays for the $\lambda$-mode $D$ wave $\Xi_{c}$ and $\Xi_{b}$ baryons. The masses of the $D$-wave $\Xi_{c}\left(\Xi_{b}\right)$ states $\left|{ }^{2} D_{\lambda \lambda^{\frac{3}{2}}}{ }^{+}\right\rangle$and $\left|{ }^{2} D_{\lambda \lambda}{ }^{\frac{5}{2}}\right\rangle$ are taken as 3055 and 3080 (6373 and 6366) $\mathrm{MeV}$, respectively. $M_{f}$ stands for the masses of $P$-wave heavy baryons $(\mathrm{MeV})$ in the final states, which are adopted from the RPP [24] and Ref. [21]. The superscript (subscript) stands for the uncertainty of a prediction with a $+10 \%(-10 \%)$ uncertainty of the oscillator parameter $\alpha_{\rho}$.

\begin{tabular}{|c|c|c|c|}
\hline & & $\left|\Xi_{c}^{2} D_{\lambda \lambda}{ }^{\frac{3}{2}}{ }^{+}\right\rangle(3055)$ & $\left|\Xi_{c}^{2} D_{\lambda \lambda \frac{5}{2}}{ }^{+}\right\rangle(3080)$ \\
\hline Decay mode & $M_{f}$ & $\Gamma_{i}(\mathrm{MeV})$ & $\Gamma_{i}(\mathrm{MeV})$ \\
\hline$\Xi_{c}^{\prime} \pi$ & 2575 & $1.93_{-0.61}^{+0.57}$ & $0.75_{+0.27}^{-0.18}$ \\
\hline$\Xi_{c}^{\prime *} \pi$ & 2645 & $0.60_{+0.07}^{-0.04}$ & $2.08_{-0.40}^{+0.38}$ \\
\hline$\Sigma_{c} K$ & 2455 & $2.49_{-0.29}^{+0.38}$ & $0.22_{+0.10}^{-0.01}$ \\
\hline$\sum_{c}^{*} K$ & 2520 & $0.14_{<+0.01}^{<-0.01}$ & $1.68_{-014}^{+0.11}$ \\
\hline$\left|\Xi_{c}^{\prime} 1^{4} P_{\lambda^{\frac{1}{2}}}-\right\rangle \pi$ & 2854 & $<0.01$ & $1.42_{-0.34}^{+0.39}$ \\
\hline$\left|\Xi_{c}^{\prime} 1^{4} P_{\lambda^{\frac{3}{2}}}^{-}\right\rangle \pi$ & 2912 & 0.04 & $0.20_{-0.03}^{+0.04}$ \\
\hline$\left|\Xi_{c}^{\prime} 1^{4} P_{\lambda^{\frac{5}{2}}-}\right\rangle \pi$ & 2929 & $\ldots$ & $0.54_{-0.10}^{+0.10}$ \\
\hline Sum & & $5.20_{-0.83}^{+0.91}$ & $6.90_{-0.64}^{+0.84}$ \\
\hline
\end{tabular}

\begin{tabular}{|c|c|c|c|}
\hline & & $\left|\Xi_{b}^{2} D_{\lambda \lambda 2^{\frac{3}{2}}}{ }^{2}\right\rangle(6366)$ & $\left|\Xi_{b}^{2} D_{\lambda \lambda \frac{5}{2}}^{+}\right\rangle(6373)$ \\
\hline Decay mode & $M_{f}$ & $\Gamma_{i}(\mathrm{MeV})$ & $\Gamma_{i}(\mathrm{MeV})$ \\
\hline$\Xi_{b}^{\prime} \pi$ & 5935 & $2.41_{-0.81}^{+0.79}$ & $0.90_{+0.20}^{-0.22}$ \\
\hline$\Xi_{b}^{\prime *} \pi$ & 5955 & $1.45_{+0.25}^{-0.13}$ & $3.22_{-0.68}^{+0.68}$ \\
\hline$\Sigma_{b} K$ & 5811 & $2.47_{-0.27}^{+0.20}$ & 0.07 \\
\hline$\Sigma_{b}^{*} K$ & 5832 & $0.30_{-0.01}^{-0.04}$ & $2.10_{-016}^{+0.12}$ \\
\hline$\left|\Xi_{b}^{\prime} 1^{4} P_{\lambda^{\frac{1}{2}}}{ }^{-}\right\rangle \pi$ & 6227 & $<0.01$ & $0.28_{-0.06}^{+0.06}$ \\
\hline$\left|\Xi_{b}^{\prime} 1^{4} P_{\lambda \frac{3}{2}}^{-}\right\rangle \pi$ & 6224 & $0.11_{-0.02}^{+0.02}$ & $0.10_{-0.02}^{+0.02}$ \\
\hline$\left|\Xi_{b}^{\prime} 1^{4} P_{\lambda^{\frac{5}{2}}-}\right\rangle \pi$ & 6226 & 0.06 & $0.45_{-0.09}^{+0.09}$ \\
\hline Sum & & $6.8_{-0.02}^{+0.92}$ & $7.11_{-0.71}^{+0.75}$ \\
\hline
\end{tabular}

Combining the predicted partial width of $\Gamma\left[\Xi_{c}(3055)^{+} \rightarrow\right.$ $\left.\Sigma_{c}(2455)^{++} K^{-}\right] \simeq 2.4 \mathrm{MeV}$ with Eq. (11), we estimate the partial width into the $\Lambda D$ channel: $\Gamma\left[\Xi_{c}(3055)^{+} \rightarrow\right.$ $\left.\Lambda D^{+}\right] \simeq 12.2 \pm 4.2 \mathrm{MeV}$. Finally, the total width of $\Xi_{c}(3055)$ is estimated to be $\Gamma \simeq 17.5 \pm 4.2 \mathrm{MeV}$, which is close to the upper limit of the observation. Other interpretations of $\Xi_{c}(3055)$ also can be found in the literature $[42,43,45]$. To further confirm the nature of $\Xi_{c}(3055)$, the ratio of $\Gamma\left[\Xi_{c}^{\prime 0} \pi^{+}\right] / \Gamma\left[\Sigma_{c}(2455)^{++} K^{-}\right]$is worth observing in future experiments.

Furthermore, the nature of $\Xi_{c}(3055)$ can be tested by its radiative decays. Assigning $\Xi_{c}(3055)$ as the $J^{P}=3 / 2^{+}$ state $\left|\Xi_{c}^{2} D_{\lambda \lambda \frac{3}{2}}{ }^{+}\right\rangle$, we study its radiative decays into the $1 P$ wave charmed baryon states. Our results are listed in Table VII. It is found that the $\Xi_{c}(3055)^{0} \rightarrow \Xi_{c}(2790)^{0} \gamma$ process has a relatively large partial decay width, $\sim 80 \mathrm{keV}$. Combining it with the measured width of $\Xi_{c}(3055)$, we predict the branching fraction $\mathcal{B}\left[\Xi_{c}(3055)^{0} \rightarrow\right.$ $\left.\Xi_{c}(2790)^{0} \gamma\right] \simeq 1.0 \%$. Thus, the neutral state $\Xi_{c}(3055)^{0}$ is most likely to be observed in the $\Xi_{c}(2790)^{0} \gamma$ channel if it corresponds to the $J^{P}=3 / 2^{+}$state $\left|\Xi_{c}{ }^{2} D_{\lambda \lambda} \lambda^{\frac{3}{2}}\right\rangle$ indeed.

\section{2. $J^{P}=5 / 2^{+}$state and $\Xi_{c}(3080)$}

The $\Xi_{c}(3080)$ resonance is suggested to be the $\rho$-mode $2 S$-wave state with $J^{P}=1 / 2^{-}$in Ref. [48]. The observation of $\Xi_{c}(3080)^{+}$in the $D^{+} \Lambda$ channel excludes this assignment because the $D^{+} \Lambda$ decay mode should be forbidden [48]. The mass and decay modes observed in experiments indicate that $\Xi_{c}(3080)$ is most likely to be the $\lambda$-mode $1 D$ excitation of $\Xi_{c}$ with $J^{P}=5 / 2^{+}$(i.e., $\left|\Xi_{c}^{2} D_{\lambda \lambda^{2}}{ }^{+}\right\rangle$) $[21,41,44]$. Considering $\Xi_{c}(3080)^{+}$as the $\left|\Xi_{c}{ }^{2} D_{\lambda \lambda^{\frac{5}{2}}}{ }^{+}\right\rangle$state, we find that it has relatively large decay rates into the $\Sigma_{c}^{*}(2520) K$ and $\Xi_{c}^{\prime *}(2645) \pi$ (see Table VI). The partial width ratio between these two main channels is predicted to be

$$
\frac{\Gamma\left[\Xi_{c}^{* 0}(2645) \pi^{+}\right]}{\Gamma\left[\Sigma_{c}(2520)^{++} K^{-}\right]} \simeq 1.2 .
$$

Combining it with the predicted partial width of $\Gamma\left[\Xi_{c}(3080)^{+} \rightarrow \Sigma_{c}(2455)^{++} K^{-}\right] \sim 0.22 \mathrm{MeV}$, we estimate that $\Gamma\left[\Xi_{c}(3080)^{+} \rightarrow \Lambda D^{+}\right] \simeq 0.2 \mathrm{MeV}$. Finally, the total width of $\Xi_{c}(3080)$ is estimated to be $\Gamma \simeq 6.9 \mathrm{MeV}$, which is close to the upper limit of the observation from the Belle Collaboration [123]. However, our predicted partial width ratio between the $\Sigma_{c} K$ and $\Sigma_{c}^{*} K$ channels

$$
\frac{\Gamma\left[\Sigma_{c}^{++}(2520) K^{-}\right]}{\Gamma\left[\Sigma_{c}(2455)^{++} K^{-}\right]} \simeq 7.6
$$

is about an order of magnitude larger than the observed ratio listed in Eq. (13), and a similar phenomenon is found by Chen et al. within their ${ }^{3} P_{0}$ analysis [41]. It should be mentioned that the measured ratio $\mathcal{R}=\frac{\Gamma\left[\Sigma_{c}^{++}(2520) K^{-}\right]}{\Gamma\left[\Sigma_{c}(2455)^{++} K^{-}\right]} \simeq$ $1.07 \pm 0.27$ of $\Xi_{c}(3080)$ may be strongly affected by its 
TABLE VII. Partial widths of radiative decays for the $\lambda$-mode $D$-wave $\Xi_{c}$ and $\Xi_{b}$ baryons. The masses of the $D$-wave $\Xi_{c}\left(\Xi_{b}\right)$ states $\left|{ }^{2} D_{\lambda \lambda^{\frac{3}{2}}}{ }^{+}\right\rangle$and $\left|{ }^{2} D_{\lambda \lambda 2^{\frac{5}{2}}}\right\rangle$ are taken as 3055 and 3080 (6366 and 6373) $\mathrm{MeV}$, respectively. $M_{f}$ stands for the masses of $P$-wave heavy baryons $(\mathrm{MeV})$ in the final states, which are adopted from the RPP [24] and Ref. [21]. The superscript (subscript) stands for the uncertainty of a prediction with a $+10 \%(-10 \%)$ uncertainty of the oscillator parameter $\alpha_{\rho}$.

\begin{tabular}{|c|c|c|c|}
\hline & & $\left|\Xi_{c}^{2} D_{\lambda \lambda}{ }^{\frac{3}{2}}{ }^{+}\right\rangle(3055)$ & $\left|\Xi_{c}{ }^{2} D_{\lambda \lambda{ }^{2}}{ }^{5}\right\rangle(3080)$ \\
\hline Decay mode & $M_{f}$ & $\Gamma_{i}(\mathrm{keV})$ & $\Gamma_{i}(\mathrm{keV})$ \\
\hline$\left|\Xi_{c}^{+2} P_{\lambda \frac{1}{2}}{ }^{-}\right\rangle \gamma$ & 2792 & $1.09_{+0.33}^{-0.22}$ & $0.36_{+0.08}^{-0.06}$ \\
\hline$\left|\Xi_{c}^{02} P_{\lambda}^{1-}{ }^{-}\right\rangle \gamma$ & & $79.0_{+16.7}^{-12.8}$ & $7.62_{+1.97}^{-1.41}$ \\
\hline$\left|\Xi_{c}^{+2} P_{\lambda \frac{3}{2}}{ }^{-}\right\rangle \gamma$ & 2815 & $0.57_{+0.15}^{-0.11}$ & $0.28_{+0.11}^{-0.07}$ \\
\hline$\left|\Xi_{c}^{02} P_{\lambda{ }^{3}}{ }^{-}\right\rangle \gamma$ & & $21.1_{+4.9}^{-3.6}$ & $85.1_{+18.2}^{-13.9}$ \\
\hline$\left|\Xi_{c}^{\prime+2} P_{\lambda \frac{1}{2}}{ }^{-}\right\rangle \gamma$ & 2936 & 0.06 & $0.26_{+0.05}^{-0.04}$ \\
\hline$\left|\Xi_{c}^{\prime 02} P_{\lambda \frac{1}{2}}{ }^{-}\right\rangle \gamma$ & & 0.0 & 0.0 \\
\hline$\left|\Xi_{c}^{\prime+2} P_{\lambda^{\frac{3}{2}}}^{-}\right\rangle \gamma$ & 2935 & $0.22_{+0.05}^{-0.04}$ & $0.42_{+0.08}^{-0.07}$ \\
\hline$\left|\Xi_{c}^{\prime 02} P_{\lambda \frac{3}{2}}{ }^{-}\right\rangle \gamma$ & & 0.0 & 0.0 \\
\hline$\left|\Xi_{c}^{\prime+4} P_{\lambda \frac{1}{2}-}{ }^{-}\right\rangle \gamma$ & 2854 & $0.97_{+0.16}^{-0.13}$ & $0.21_{+0.03}^{-0.02}$ \\
\hline$\left|\Xi_{c}^{\prime 04} P_{\lambda 2^{\frac{1}{2}}-}\right\rangle \gamma$ & & 0.0 & 0.0 \\
\hline$\left|\Xi_{c}^{\prime+4} P_{\lambda \frac{3}{2}}^{-}\right\rangle \gamma$ & 2912 & $0.66_{+0.13}^{-0.11}$ & $0.63_{+0.12}^{-0.10}$ \\
\hline$\left|\Xi_{c}^{\prime 04} P_{\lambda 2^{\frac{3}{2}}}\right\rangle \gamma$ & & 0.0 & 0.0 \\
\hline$\left|\Xi_{c}^{\prime+4} P_{\lambda{ }^{\frac{5}{2}}}\right\rangle \gamma$ & 2929 & 0.09 & $1.24_{+0.27}^{-0.20}$ \\
\hline$\left|\Xi_{c}^{\prime 04} P_{\lambda 2^{\frac{5}{2}}}\right\rangle \gamma$ & & 0.0 & 0.0 \\
\hline
\end{tabular}

\begin{tabular}{|c|c|c|c|}
\hline & & $\left|\Xi_{b}^{2} D_{\lambda \lambda 2^{3}}{ }^{3}\right\rangle(6366)$ & $\left|\Xi_{b}{ }^{2} D_{\lambda \lambda{ }^{2}}{ }^{+}\right\rangle(6373)$ \\
\hline Decay mode & $M_{f}$ & $\Gamma_{i}(\mathrm{keV})$ & $\Gamma_{i}(\mathrm{keV})$ \\
\hline$\left|\Xi_{b}^{02} P_{\lambda{ }^{\frac{1}{2}}-}\right\rangle \gamma$ & 6120 & $3.62_{+0.64}^{-0.51}$ & $0.33_{+0.10}^{-0.07}$ \\
\hline$\left|\Xi_{b}^{-2} P_{\lambda{ }^{1}}^{1-}\right\rangle \gamma$ & & $32.0_{+6.2}^{-4.9}$ & $2.58_{+0.76}^{-0.53}$ \\
\hline$\left|\Xi_{b}^{02} P_{\lambda^{\frac{3}{2}}}^{-}\right\rangle \gamma$ & 6130 & $1.09_{+0.26}^{-0.19}$ & $4.78_{+0.81}^{-0.71}$ \\
\hline$\left|\Xi_{b}^{-2} P_{\lambda 2^{\frac{3}{2}}}\right\rangle \gamma$ & & $9.40_{+2.19}^{-1.63}$ & $39.5_{+7.9}^{-6.2}$ \\
\hline$\left|\Xi_{b}^{\prime 02} P_{\lambda}{ }^{\frac{1}{2}}{ }^{-}\right\rangle \gamma$ & 6233 & $0.17_{+0.04}^{-0.02}$ & $0.37_{+0.07}^{-0.05}$ \\
\hline$\left|\Xi_{b}^{\prime-2} P_{\lambda \frac{1}{2}-}\right\rangle \gamma$ & & 0.0 & 0.0 \\
\hline$\left.\left|\Xi_{b}^{\prime 02} P_{\lambda \frac{3}{2}}\right\rangle^{-}\right\rangle$ & 6234 & $0.57_{+0.13}^{-0.09}$ & $0.56_{+0.12}^{-0.09}$ \\
\hline$\left|\Xi_{b}^{\prime-2} P_{\lambda \frac{3}{2}}{ }^{-}\right\rangle \gamma$ & & 0.0 & 0.0 \\
\hline$\left|\Xi_{b}^{\prime 04} P_{\lambda \frac{1}{2}}{ }^{-}\right\rangle \gamma$ & 6227 & $0.31_{+0.05}^{-0.05}$ & 0.05 \\
\hline$\left|\Xi_{b}^{\prime-4} P_{\lambda} \frac{1}{2}-\right\rangle \gamma$ & & 0.0 & 0.0 \\
\hline$\left|\Xi_{b}^{\prime 04} P_{\lambda \frac{3}{2}}{ }^{-}\right\rangle \gamma$ & 6224 & $1.04_{+0.22}^{-0.16}$ & $0.59_{+0.11}^{-0.08}$ \\
\hline$\left|\Xi_{b}^{\prime-4} P_{\lambda \frac{3}{2}}{ }^{-}\right\rangle \gamma$ & & 0.0 & 0.0 \\
\hline$\left|\Xi_{b}^{\prime 04} P_{\lambda{ }^{5}}{ }^{-}\right\rangle \gamma$ & 6226 & $0.27_{+0.06}^{-0.04}$ & $1.82_{+0.38}^{-0.29}$ \\
\hline$\left|\Xi_{b}^{\prime-4} P_{\lambda \frac{5}{2}-}\right\rangle \gamma$ & & 0.0 & 0.0 \\
\hline
\end{tabular}

nearby states, such as $\Xi_{c}(3055)$. Thus, the measured ratio from Belle [123] may not be a genuine ratio for $\Xi_{c}(3080)$.

Furthermore, assigning $\Xi_{c}(3080)$ as the $J^{P}=5 / 2^{+}$state $\left|\Xi_{c}^{2} D_{\lambda \lambda^{2}}{ }^{+}\right\rangle$, we study its radiative decays. Our results are listed in Table VII. It is found that the $\Xi_{c}(3080)^{0}$ should have a relatively large decay rate into $\Xi_{c}(2815)^{03}{ }^{2}-\gamma$. The partial decay width is predicted to be $\Gamma\left[\Xi_{c}(3080)^{0} \rightarrow\right.$ $\left.\Xi_{c}(2815)^{0} \gamma\right] \simeq 85 \mathrm{keV}$. Combining it with the measured width of $\Xi_{c}(3080)$, we predict the branching fraction $\mathcal{B}\left[\Xi_{c}(3055)^{0} \rightarrow \Xi_{c}(2815)^{0} \gamma\right] \simeq 3 \%$. The neutral state $\Xi_{c}(3080)^{0}$ is most likely to be observed in the $\Xi_{c}(2815)^{0} \gamma$ channel if it corresponds to the $J^{P}=5 / 2^{+}$ state $\left|\Xi_{c}^{2} D_{\lambda \lambda^{2}}{ }^{+}\right\rangle$indeed.

\section{D. $\Xi_{b}$ states}

In the $\Xi_{b}$ family, there are two $\lambda$-mode $1 D$-wave

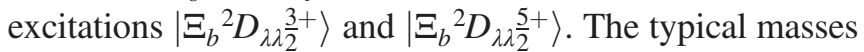
of the $\lambda$-mode $1 D$-wave $\Xi_{b}$ excitations are $6.3-6.4 \mathrm{GeV}$ within various quark model predictions (see Table I). In the possible mass regions, the strong decays of these $1 D$-wave states are studied with ChQM. Our results have been shown in Fig. 2. To be more specific, taking the masses of the $1 D$-wave states obtained in the relativistic quark-diquark picture [21], we give the predicted widths in Table VI.

\section{1. $J^{P}=3 / 2^{+}$state}

The $J^{P}=3 / 2^{+}$state $\left|\Xi_{b}{ }^{2} D_{\lambda \lambda^{2}}{ }^{+}\right\rangle$might be a narrow state with a width of a few $\mathrm{MeV}$. It mainly decays into $\Xi_{b}^{\prime} \pi, \Xi_{b}^{\prime *} \pi$ and $\Sigma_{b} K$ channels. The partial widths of $\Xi_{b}^{\prime} \pi, \Xi_{b}^{\prime *} \pi$ are less sensitive to the mass of $\left|\Xi_{b}{ }^{2} D_{\lambda \lambda 2^{2}}{ }^{+}\right\rangle$; however, the partial width for the $\Sigma_{b} K$ channel shows a significant linear dependence on the mass (see Fig. 2). If the mass of $\left|\Xi_{b}^{2} D_{\lambda 2^{2}}{ }^{+}\right\rangle$takes the predicted value $\sim 6.37 \mathrm{GeV}$ in Ref. [21], the branching fractions for the main channels are predicted to be

$$
\frac{\Gamma\left[\Xi_{b}^{\prime} \pi, \Xi_{b}^{\prime *} \pi, \Sigma_{b} K\right]}{\Gamma_{\text {total }}}=35 \%, 21 \%, 36 \% .
$$

The $\Xi_{b}^{\prime} \pi$ and $\Sigma_{b} K$ decay channels may be ideal channels for our search for this missing $1 D$-wave $\Xi_{b}$ baryon in future experiments.

Furthermore, we study the radiative decays of $\left|\Xi_{b}^{2} D_{\lambda \lambda^{2}}{ }^{+}\right\rangle$into the $1 P$-wave bottom baryon states. Our results are listed in Table VII. It is found that the charged state $\left|\Xi_{b}^{-2} D_{\lambda \lambda^{3}}{ }^{+}\right\rangle$might have a relatively large decay rate into $\Xi_{b}^{-}\left(\frac{1}{2}\right) \gamma$. The partial decay width can reach up to $\sim 30 \mathrm{keV}$ if the mass for $\left|\Xi_{b}{ }^{2} D_{\lambda \lambda \frac{3}{2}}{ }^{+}\right\rangle$is taken to be $\sim 6366 \mathrm{MeV}$ as predicted in the relativistic quark model [21]. Combining the predicted total width of $\left|\Xi_{b}{ }^{2} D_{\lambda \lambda \frac{3}{2}}{ }^{+}\right\rangle$, we estimate the branching fraction $\mathcal{B}\left[\left|\Xi_{b}^{-2} D_{\lambda \lambda \lambda^{\frac{3}{2}}}\right\rangle \rightarrow\right.$ $\left.\Xi_{b}^{-}\left(\frac{1}{2}^{-}\right) \gamma\right] \simeq \mathcal{O}\left(10^{-3}\right)$.

\section{2. $J^{P}=5 / 2^{+}$state}

The $J^{P}=5 / 2^{+}$state $\left|\Xi_{b}^{2} D_{\lambda \lambda^{2}}{ }^{+}\right\rangle$may be a narrow state with a width comparable to the $J^{P}=3 / 2^{+}$state $\left|\Xi_{b}{ }^{2} D_{\lambda \lambda^{\frac{3}{2}}}{ }^{+}\right\rangle$ (i.e., a few MeV). The decays of $\left|\Xi_{b}{ }^{2} D_{\lambda \lambda^{2}}{ }^{+}\right\rangle$are governed by $\Xi_{b}^{\prime *} \pi$, which is less sensitive to its mass. If the mass of $\left|\Xi_{b}{ }^{2} D_{\lambda \lambda 2^{2}}{ }^{+}\right\rangle$is taken to be $\sim 6.37 \mathrm{GeV}$ as the prediction in Ref. [21], the decay channel $\Sigma_{b}^{*} K$ becomes important as well (see Table VI). In this case, the branching fractions for the $\Xi_{b}^{\prime} \pi, \Xi_{b}^{\prime *} \pi$ and $\Sigma_{b} K$ channels are predicted to be 


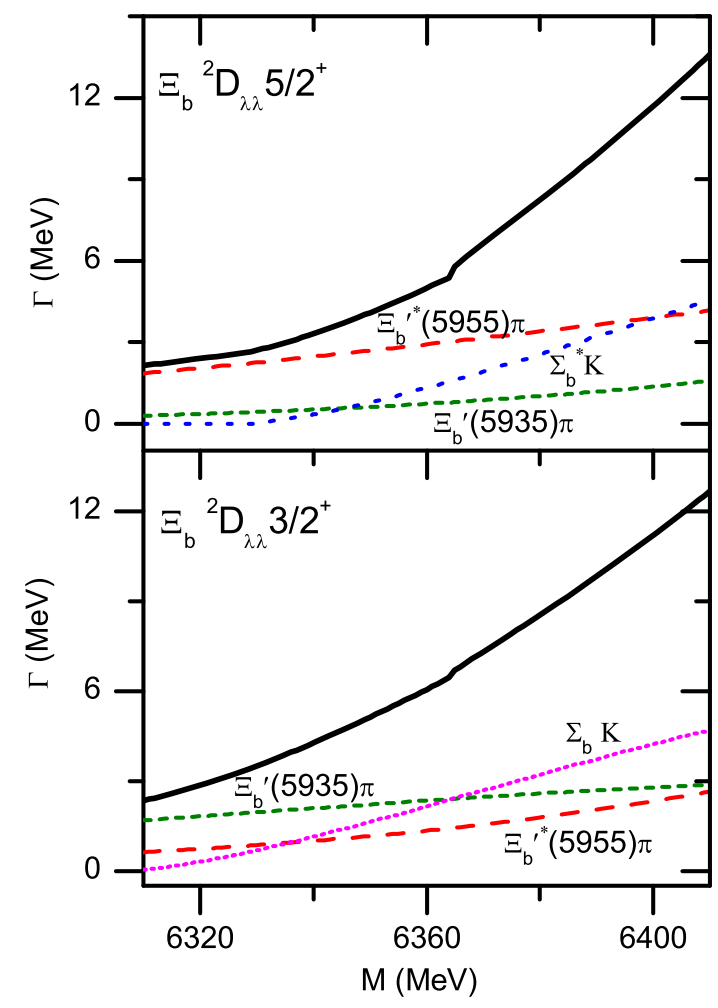

FIG. 2. Strong decay partial widths of the main decay modes for the $\lambda$-mode $1 D$-wave excited $\Xi_{b}$ states $\left|\Xi_{b} D_{\lambda \lambda} 3 / 2^{+}\right\rangle$and $\left|\Xi_{b} D_{\lambda \lambda} 5 / 2^{+}\right\rangle$as functions of their mass. The solid curves stand for the sum of the strong decay partial widths.

$$
\frac{\Gamma\left[\Xi_{b}^{\prime} \pi, \Xi_{b}^{\prime *} \pi, \Sigma_{b} K\right]}{\Gamma_{\text {total }}}=12 \%, 45 \%, 29 \%
$$

To establish this missing $1 D$-wave $\Xi_{b}$ baryon with $J^{P}=5 / 2^{+}$, its dominant decay modes $\Xi_{b}^{\prime *} \pi$ and $\Sigma_{b} K$ are worth observing in future experiments.

We also study the radiative decays of $\left|\Xi_{b}{ }^{2} D_{\lambda \lambda \frac{5}{2}}{ }^{+}\right\rangle$into the $1 P$-wave bottom baryon states. Our results are listed in Table VII as well. It is found that the charged state $\left|\Xi_{b}^{-2} D_{\lambda \lambda \frac{5}{2}}{ }^{+}\right\rangle$might have a relatively large decay rate into $\Xi_{b}^{-}\left(\frac{3}{2}\right) \gamma$. The partial decay width can reach up to $\sim 40 \mathrm{keV}$. If the mass for $\left|\Xi_{b}{ }^{2} D_{\lambda \lambda^{\frac{3}{2}}}{ }^{+}\right\rangle$is taken to be $\sim 6373 \mathrm{MeV}$ as predicted in the relativistic quark model [21]. Combining the predicted total width of $\left|\Xi_{b}{ }^{2} D_{\lambda \lambda^{2}}{ }^{+}\right\rangle$, we estimate the branching fraction $\mathcal{B}\left[\left|\Xi_{b}^{-2} D_{\lambda \lambda 2^{\frac{5}{2}}}\right\rangle \rightarrow \Xi_{b}^{-}\left(\frac{3}{2}^{-}\right) \gamma\right] \simeq \mathcal{O}\left(10^{-3}\right)$.

\section{RESULTS FOR SINGLY HEAVY BARYONS OF $6_{F}$}

\section{A. $\Sigma_{c}$}

In the $\Sigma_{c}$ family, according to the quark model classification, there are six $\lambda$-mode $1 D$-wave excitations: $\left|\Sigma_{c}{ }^{4} D_{\lambda \lambda \lambda^{\frac{1}{2}}}\right\rangle,\left|\Sigma_{c}{ }^{4} D_{\lambda \lambda^{\frac{3}{2}}}{ }^{+}\right\rangle,\left|\Sigma_{c}{ }^{2} D_{\lambda \lambda^{2}}{ }^{+}\right\rangle,\left|\Sigma_{c}{ }^{2} D_{\lambda \lambda \lambda^{2}}{ }^{+}\right\rangle$, $\left|\Sigma_{c}{ }^{4} D_{\lambda \lambda^{\frac{5}{2}}}{ }^{+}\right\rangle$, and $\left|\Sigma_{c}{ }^{4} D_{\lambda \lambda^{\frac{7}{2}}}{ }^{+}\right\rangle$. However, no $D$-wave states have been established. The typical masses of the $\lambda$-mode $1 D$-wave $\Sigma_{c}$ excitations are predicted to be $\sim 3.0 \mathrm{GeV}$ within various quark models (see Table II). In the possible
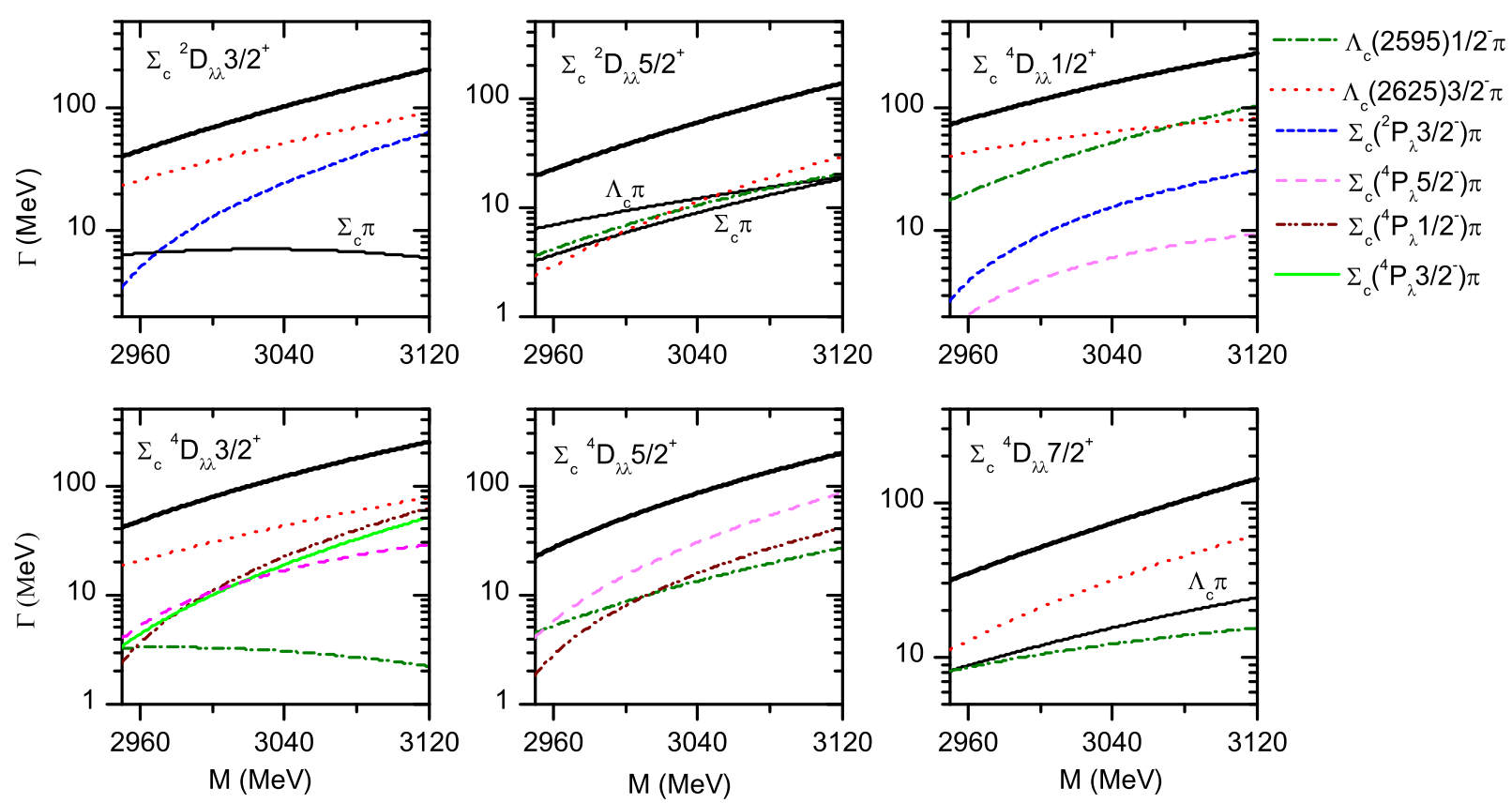

FIG. 3. Strong decay partial widths of the main decay modes for the $\lambda$-mode $1 D$-wave excited $\Sigma_{c}$ states as functions of their mass. The bold solid curves stand for the sum of the partial widths. The masses of the $P$-wave heavy baryons in final states are adopted from the quark model predictions in Ref. [21] (see Table II). 
TABLE VIII. Strong decay partial widths of the main decay modes for the $\lambda$-mode $D$-wave $\Sigma_{c}$ and $\Sigma_{b}$ baryons, the masses (MeV) of which taken from the quark model predictions of Ref. [21]. $M_{f}$ stands for the masses of $P$-wave heavy baryons (MeV) in the final states, which are adopted from the RPP [24] and Ref. [21]. The superscript (subscript) stands for the uncertainty of a prediction with a $+10 \%$ $(-10 \%)$ uncertainty of the oscillator parameter $\alpha_{\rho}$.

\begin{tabular}{|c|c|c|c|c|c|c|c|}
\hline & & $\left|\Sigma_{c}^{2} D_{\lambda \lambda{ }^{2}}{ }^{+}\right\rangle(3043)$ & $\left|\Sigma_{c}^{2} D_{\lambda \lambda \frac{5}{2}}{ }^{+}\right\rangle(3038)$ & $\left|\Sigma_{c}^{4} D_{\lambda \lambda{ }^{2}}{ }^{+}\right\rangle(3041)$ & $\left|\Sigma_{c}{ }^{4} D_{\lambda \lambda \frac{3}{2}}{ }^{+}\right\rangle(3040)$ & $\left|\Sigma_{c}{ }^{4} D_{\lambda \lambda}{ }^{\frac{5}{2}}\right\rangle(3023)$ & $\mid \Sigma_{c}^{4} D_{\left.\lambda \lambda \frac{7}{2}{ }^{+}\right\rangle(3013)}$ \\
\hline Decay mode & $M_{f}$ & $\Gamma_{i}(\mathrm{MeV})$ & $\Gamma_{i}(\mathrm{MeV})$ & $\Gamma_{i}(\mathrm{MeV})$ & $\Gamma_{i}(\mathrm{MeV})$ & $\Gamma_{i}(\mathrm{MeV})$ & $\Gamma_{i}(\mathrm{MeV})$ \\
\hline$\Lambda_{c} \pi$ & 2286 & $1.29_{-1.22}^{+2.54}$ & $11.9_{+1.51}^{-1.65}$ & $2.62_{-2.46}^{+5.09}$ & $1.15_{-1.09}^{+2.32}$ & $3.1_{+0.42}^{-0.46}$ & $13.1_{+1.83}^{-2.06}$ \\
\hline$\Sigma_{c} \pi$ & 2455 & $7.06_{-3.95}^{+4.66}$ & $8.78_{+2.25}^{-1.86}$ & $3.53_{-1.96}^{+2.31}$ & $1.77_{-0.98}^{+1.14}$ & $0.54_{+0.14}^{-0.11}$ & $2.18_{+0.60}^{-0.48}$ \\
\hline$\sum_{c}^{*} \pi$ & 2520 & $2.44_{+0.48}^{-0.33}$ & $2.91_{-0.61}^{+0.72}$ & $1.71_{-0.76}^{+0.79}$ & $7.47_{-1.77}^{+2.04}$ & $11.6_{-0.34}^{+0.80}$ & $1.45_{-0.02}^{+0.00}$ \\
\hline$\Xi_{c} K$ & 2470 & $1.17_{-0.14}^{+0.12}$ & 0.03 & $2.27_{-0.27}^{+0.22}$ & $1.12_{-0.13}^{+0.10}$ & $<0.01$ & 0.01 \\
\hline$\left|\Lambda_{c}^{2} P_{\lambda{ }^{2}}{ }^{\frac{1}{2}}\right\rangle \pi$ & 2592 & $4.93_{-0.19}^{+0.25}$ & $10.3_{+0.68}^{-0.83}$ & $52.6_{+2.7}^{-3.2}$ & $3.06_{-1.70}^{+2.44}$ & $11.2_{+0.9}^{-0.8}$ & $11.1_{-3.0}^{+3.5}$ \\
\hline$\left|\Lambda_{c}^{2} P_{\lambda{ }^{2}}^{3-}\right\rangle \pi$ & 2628 & $52.8_{-5.77}^{+6.76}$ & $10.9_{-2.4}^{+2.1}$ & $64.0_{-18.8}^{+22.2}$ & $43.1_{-3.7}^{+4.4}$ & $1.92_{<+0.01}^{+0.02}$ & $24.2_{-0.04}^{+0.05}$ \\
\hline$\left|\Sigma_{c}^{2} P_{\lambda}^{\frac{1}{2}}{ }^{-}\right\rangle \pi$ & 2713 & $4.09_{-0.29}^{+0.35}$ & $5.85_{+0.86}^{-0.75}$ & $7.56_{+0.89}^{-0.77}$ & $2.10_{-0.82}^{+0.99}$ & $1.13_{+0.18}^{-0.16}$ & $2.83_{-0.68}^{+0.76}$ \\
\hline$\left|\Sigma_{c}^{2} P_{\lambda}{ }^{\frac{3}{2}}-\right\rangle \pi$ & 2798 & $25.6_{-4.29}^{+4.82}$ & $0.76_{+0.18}^{-0.05}$ & $15.8_{-3.6}^{+4.0}$ & $3.58_{-0.34}^{+0.39}$ & 0.01 & $0.95_{-0.04}^{+0.06}$ \\
\hline$\left|\Sigma_{c}{ }^{4} P_{\lambda \frac{1}{2}}^{1-}\right\rangle \pi$ & 2799 & 0.09 & $1.30_{-0.33}^{+0.38}$ & $0.46_{+0.10}^{-0.07}$ & $22.4_{-3.0}^{+3.4}$ & $12.1_{-1.9}^{+2.1}$ & $<0.01$ \\
\hline$\left|\Sigma_{c}{ }^{4} P_{\lambda{ }^{3}}{ }^{-}\right\rangle \pi$ & 2773 & $3.42_{-0.16}^{+0.22}$ & $1.26_{-0.09}^{+0.11}$ & $4.18_{+0.02}^{+0.05}$ & $18.9_{-2.0}^{+2.4}$ & $4.54_{-1.80}^{+2.16}$ & $1.03_{+0.04}^{-0.03}$ \\
\hline$\left|\Sigma_{c}{ }^{4} P_{\lambda \overline{2}^{-}}-\right\rangle \pi$ & 2789 & $2.20_{<+0.01}^{+0.06}$ & $4.51_{-0.45}^{+0.57}$ & $6.1_{-1.72}^{+1.96}$ & $16.8_{-4.3}^{+4.8}$ & $23.0_{-2.9}^{+3.3}$ & $1.26_{+0.09}^{-0.05}$ \\
\hline \multirow[t]{2}{*}{ Sum } & & $105_{-15.5}^{+19.5}$ & $58.5_{+1.6}^{-1.3}$ & $160.8_{-25.9}^{+32.6}$ & $121.5_{-19.8}^{+24.4}$ & $69.1_{-5.3}^{+6.9}$ & $58.1_{-1.2}^{+1.9}$ \\
\hline & & $\left|\Sigma_{b}^{2} D_{\lambda \lambda \lambda^{\frac{3}{2}}}\right\rangle(6326)$ & $\left|\Sigma_{b}^{2} D_{\lambda \lambda \frac{5}{2}}{ }^{+}\right\rangle(6284)$ & $\left|\Sigma_{b}^{4} D_{\lambda \lambda{ }^{2}}{ }^{+}\right\rangle(6311)$ & $\left|\Sigma_{b}^{4} D_{\lambda \lambda{ }^{\frac{3}{2}}}\right\rangle(6285)$ & $\left|\Sigma_{b}{ }^{4} D_{\lambda \lambda}{ }^{\frac{5}{2}}\right\rangle(6270)$ & $\left|\Sigma_{b}{ }^{4} D_{\lambda \lambda \frac{2}{2}}{ }^{7+}\right\rangle(6260)$ \\
\hline Decay mode & $M_{f}$ & $\Gamma_{i}(\mathrm{MeV})$ & $\Gamma_{i}(\mathrm{MeV})$ & $\Gamma_{i}(\mathrm{MeV})$ & $\Gamma_{i}(\mathrm{MeV})$ & $\Gamma_{i}(\mathrm{MeV})$ & $\Gamma_{i}(\mathrm{MeV})$ \\
\hline$\Lambda_{b} \pi$ & 5620 & $0.56_{-0.46}^{+2.76}$ & $17.3_{+1.6}^{-1.1}$ & $1.56_{-1.51}^{+5.81}$ & $1.21_{-1.20}^{+3.07}$ & $4.43_{+0.45}^{-0.58}$ & $18.5_{+2.1}^{-2.6}$ \\
\hline$\Sigma_{b} \pi$ & 5811 & $8.28_{-4.66}^{+5.54}$ & $6.32_{+1.82}^{-1.44}$ & $4.16_{-2.20}^{+2.51}$ & $2.03_{-0.96}^{+1.03}$ & $0.37_{+0.11}^{-0.09}$ & $1.43_{+0.46}^{-0.33}$ \\
\hline$\Sigma_{b}^{*} \pi$ & 5832 & $4.21_{+0.80}^{-0.58}$ & $3.26_{-0.70}^{+0.81}$ & $2.05_{-1.00}^{+1.08}$ & $8.36_{-2.01}^{+2.26}$ & $12.6_{-0.5}^{+0.9}$ & $1.54_{-0.05}^{+0.15}$ \\
\hline$\Xi_{b} K$ & 5794 & $0.89_{-0.06}^{+0.06}$ & $\ldots$ & $0.88_{-0.04}^{+0.03}$ & $\ldots$ & $\ldots$ & $\ldots$ \\
\hline$\left|\Lambda_{b}^{2} P_{\lambda \frac{1}{2}-}\right\rangle \pi$ & 5912 & $5.70_{-0.13}^{+0.22}$ & $6.96_{+0.54}^{-0.61}$ & $51.6_{+2.2}^{-2.9}$ & $3.05_{-1.55}^{+2.13}$ & $7.68_{+0.66}^{-0.65}$ & $8.89_{-2.34}^{+2.77}$ \\
\hline$\left|\Lambda_{b}^{2} P_{\lambda{ }^{2}}^{\frac{3}{2}}\right\rangle \pi$ & 5920 & $70.0_{-7.5}^{+8.3}$ & $10.4_{+2.3}^{-2.0}$ & $66.0_{-20.1}^{+24.1}$ & $40.5_{-3.4}^{+4.1}$ & $1.90_{+0.02}^{-0.001}$ & $21.1_{-0.1}^{+0.06}$ \\
\hline$\left|\Sigma_{b}^{2} P_{\lambda{ }^{2}}^{1-}\right\rangle \pi$ & 6101 & $1.18_{-0.13}^{+0.15}$ & $0.13_{+0.03}^{-0.02}$ & $0.71_{+0.09}^{-0.07}$ & $0.81_{-0.20}^{+0.22}$ & 0.01 & $0.43_{-0.08}^{+0.09}$ \\
\hline$\left|\Sigma_{b}^{2} P_{\lambda{ }^{\frac{3}{2}}}-\right\rangle \pi$ & 6096 & $25.6_{-4.2}^{+4.8}$ & $0.28_{-0.05}^{+0.09}$ & $13.1_{-2.9}^{+3.2}$ & $1.49_{-0.18}^{+0.20}$ & $<0.01$ & $0.24_{-0.03}^{+0.03}$ \\
\hline$\left|\Sigma_{b}{ }^{4} P_{\lambda{ }^{\frac{1}{2}}}-\right\rangle \pi$ & 6095 & $0.10_{+0.07}^{-0.04}$ & $0.79_{-0.18}^{+0.21}$ & $0.33_{+0.07}^{-0.06}$ & $10.5_{-1.55}^{+1.81}$ & $5.30_{-0.90}^{+1.0}$ & $<0.01$ \\
\hline$\left|\Sigma_{b}{ }^{4} P_{\lambda{ }^{\frac{3}{2}}-}\right\rangle \pi$ & 6087 & $2.53_{-0.12}^{+0.16}$ & $0.41_{-0.05}^{+0.05}$ & $2.24_{-0.01}^{+0.04}$ & $6.44_{-0.88}^{+1.01}$ & $3.52_{-0.97}^{+1.09}$ & $0.15_{<-0.01}^{+0.01}$ \\
\hline$\left|\Sigma_{b}{ }^{4} P_{\lambda \overline{2}^{-}}\right\rangle \pi$ & 6084 & $2.39_{+0.03}^{+0.02}$ & $2.09_{-0.27}^{+0.32}$ & $5.46_{-1.52}^{+1.72}$ & $10.7_{-2.5}^{+2.8}$ & $10.8_{-1.6}^{+1.8}$ & $0.34_{-0.01}^{+0.02}$ \\
\hline Sum & & $121.4_{-16.4}^{+21.4}$ & $47.9_{+5.0}^{-3.7}$ & $148.0_{-26.9}^{+35.5}$ & $85.1_{-14.4}^{+18.6}$ & $48.5_{-2.7}^{+3.5}$ & $52.6_{-0.05}^{+0.2}$ \\
\hline
\end{tabular}

mass range, we study their strong decay transitions within ChQM. Our results are shown in Fig. 3. To be more specific, taking the masses of the $1 D$-wave states as predicted in the relativistic quark-diquark picture [21], we further present the results in Table VIII.

\section{1. $J^{P}=1 / 2^{+}$state}

The $J^{P}=1 / 2^{+}$state $\left|\Sigma_{c}{ }^{4} D_{\lambda \lambda^{2}}{ }^{+}\right\rangle$might be a broad state. If its mass is taken as the prediction $3041 \mathrm{MeV}$ in Ref. [21], the sum of the partial widths for the pionic and kaonic decays can reach up to $\Gamma_{\text {Sum }} \sim 160 \mathrm{MeV}$ (see Table VIII). This state has large decay rates into $\Lambda_{c}(2595) \pi$ and $\Lambda_{c}(2625) \pi$ final states. The ratios between the partial decay widths for the $\Lambda_{c}(2595) \pi$ and $\Lambda_{c}(2625) \pi$ channels and $\Gamma_{\text {Sum }}$ are predicted to be
$\frac{\Gamma\left[\Lambda_{c}(2595) \pi\right]}{\Gamma_{\text {Sum }}} \simeq 33 \%, \quad \frac{\Gamma\left[\Lambda_{c}(2625) \pi\right]}{\Gamma_{\text {Sum }}} \simeq 40 \%$

Both $\Lambda_{c}(2595) \pi$ and $\Lambda_{c}(2625) \pi$ may be ideal channels for our search for $\left|\Sigma_{c}{ }^{4} D_{\lambda \lambda} \lambda^{\frac{1}{2}}\right\rangle$ in future experiments.

We also estimate its radiative transitions into the $1 P$-wave charmed baryon states. Our results are listed in Table IX. It is found that $\left|\Sigma_{c}^{++(0)}{ }^{4} D_{\lambda \lambda \frac{1}{2}}{ }^{+}\right\rangle$might have relatively large decay rates into $\left|\Sigma_{c}^{++(0)}{ }^{4} P_{\lambda{ }^{2}}{ }^{-}\right\rangle \gamma$ and $\left|\Sigma_{c}^{++(0)}{ }^{4} P_{\lambda^{\frac{3}{2}}}{ }^{-}\right\rangle \gamma$, and their partial radiative decay widths are estimated to be $\mathcal{O}(10)-\mathcal{O}(100) \mathrm{keV}$. The branching fractions for these main radiative decay processes may reach up to $\mathcal{O}\left(10^{-4}\right)-\mathcal{O}\left(10^{-3}\right)$. 
TABLE IX. Partial widths of radiative decays for the $\lambda$-mode $D$-wave $\Sigma_{c}$ and $\Sigma_{b}$ baryons, the masses (MeV) of which are taken from the quark model predictions of Ref. [21]. $M_{f}$ stands for the masses of $P$-wave heavy baryons $(\mathrm{MeV})$ in the final states, which are adopted from the RPP [24] and Ref. [21]. The superscript (subscript) stands for the uncertainty of a prediction with a $+10 \%(-10 \%)$ uncertainty of the oscillator parameter $\alpha_{\rho}$.

\begin{tabular}{|c|c|c|c|c|c|c|c|}
\hline & & $\left|\Sigma_{c}^{2} D_{\lambda \lambda}{ }^{\frac{3}{2}}\right\rangle(3043)$ & $\left|\Sigma_{c}^{2} D_{\lambda \lambda{ }^{2}}{ }^{+}\right\rangle(3038)$ & $\left|\Sigma_{c}^{4} D_{\lambda \lambda \frac{1}{2}}{ }^{+}\right\rangle(3041)$ & $\left|\Sigma_{c}^{4} D_{\lambda \lambda \frac{3}{2}}{ }^{+}\right\rangle(3040)$ & $\left|\Sigma_{c}{ }^{4} D_{\lambda \lambda \frac{5}{2}}{ }^{+}\right\rangle(3023)$ & $\left|\Sigma_{c}{ }^{4} D_{\lambda \lambda{ }^{2}}{ }^{+}\right\rangle(3013)$ \\
\hline Decay mode & $M_{f}$ & $\Gamma_{i}(\mathrm{keV})$ & $\Gamma_{i}(\mathrm{keV})$ & $\Gamma_{i}(\mathrm{keV})$ & $\Gamma_{i}(\mathrm{keV})$ & $\Gamma_{i}(\mathrm{keV})$ & $\Gamma_{i}(\mathrm{keV})$ \\
\hline$\left|\Sigma_{c}^{++2} P_{\lambda \frac{1}{2}-}\right\rangle \gamma$ & 2713 & $231.9_{+30.6}^{-27.7}$ & $58.4_{+8.1}^{-7.1}$ & $15.01_{+2.3}^{-1.9}$ & $23.6_{+2.9}^{-2.7}$ & $7.40_{+1.04}^{-0.92}$ & 0.017 \\
\hline$\left|\Sigma_{c}^{+2} P_{\lambda^{\frac{1}{2}}}{ }^{-}\right\rangle \gamma$ & & $1.50_{-0.08}^{-0.01}$ & $2.27_{+0.19}^{-0.1}$ & $2.56_{+0.46}^{-0.37}$ & $2.58_{+0.37}^{-0.33}$ & $0.80_{+0.13}^{-0.11}$ & 0.001 \\
\hline $\mid \Sigma_{c}^{02} P_{\lambda} \frac{1}{2}$ & & $164.2_{+28.2}^{-23.2}$ & $22.6_{+4.0}^{-3.1}$ & $0.45_{+0.02}^{+0.40}$ & $2.71_{+0.22}^{-0.24}$ & $0.87_{+0.09}^{-0.13}$ & 0.004 \\
\hline$\left|\Sigma_{c}^{++2} P_{\lambda \frac{3}{2}^{-}}\right\rangle \gamma$ & 2798 & $78.5_{-15.1}^{+12.0}$ & $34.9_{+7.1}^{-5.5}$ & $1.93_{+0.36}^{-0.29}$ & $0.59_{+0.13}^{-0.10}$ & $4.90_{+0.85}^{-0.69}$ & $3.94_{+0.67}^{-0.55}$ \\
\hline$\left|\Sigma_{c}^{+2} P_{\lambda^{\frac{3}{2}}}{ }^{-}\right\rangle \gamma$ & & $1.82_{+0.28}^{-0.1}$ & $0.81_{+0.18}^{-0.14}$ & $0.20_{+0.04}^{-0.03}$ & 0.06 & $0.52_{+0.09}^{-0.08}$ & $0.42_{+0.07}^{-0.07}$ \\
\hline$\left|\Sigma_{c}^{02} P_{\lambda 2^{3}}{ }^{-}\right\rangle \gamma$ & & $38.6_{+8.0}^{-6.1}$ & $47.52_{+10.6}^{-8.0}$ & $0.24_{+0.04}^{-0.03}$ & 0.08 & $0.60_{+0.09}^{-0.08}$ & $0.49_{+0.07}^{-0.07}$ \\
\hline$\left|\Sigma_{c}^{++4} P_{\lambda \frac{1}{2}}{ }^{-}\right\rangle \gamma$ & 2799 & $5.09_{+0.70}^{-0.63}$ & $0.29_{+0.02}^{-0.03}$ & $136.5_{+23.5}^{-19.3}$ & $52.7_{+9.4}^{-7.6}$ & $\begin{array}{l}9.79_{+1.88}^{-1.48} \\
\end{array}$ & $1.30_{+0.41}^{-0.28}$ \\
\hline$\left|\Sigma_{c}^{+4} P_{\lambda^{\frac{1}{2}}}{ }^{-}\right\rangle \gamma$ & & $0.55_{+0.08}^{-0.08}$ & 0.03 & $0.97_{+0.06}^{-0.07}$ & $0.45_{+0.05}^{-0.05}$ & $0.14_{+0.02}^{-0.01}$ & 0.005 \\
\hline$\left|\Sigma_{c}^{04} P_{\lambda} \frac{1}{2}^{-}\right\rangle \gamma$ & & $0.60_{+0.06}^{-0.06}$ & 0.03 & $94.3_{+18.4}^{-14.5}$ & $38.61_{+7.8}^{-6.0}$ & $5.64_{+1.17}^{-0.90}$ & $1.00_{+0.28}^{-0.20}$ \\
\hline$\left|\Sigma_{c}^{++4} P_{\lambda{ }^{3}}{ }^{-}\right\rangle \gamma$ & 2773 & $11.9_{+1.8}^{-1.6}$ & $4.73_{+0.59}^{-0.54}$ & $94.6_{+16.5}^{-13.6}$ & $121.8_{+20.5}^{-17.0}$ & $48.5_{+8.9}^{-7.2}$ & $13.5_{+2.5}^{-2.0}$ \\
\hline$\left|\Sigma_{c}^{+4} P_{\lambda^{\frac{3}{2}}}{ }^{-}\right\rangle \gamma$ & & $1.27_{+0.21}^{-0.17}$ & $0.51_{+0.08}^{-0.06}$ & $1.26_{+0.14}^{-0.13}$ & $0.90_{+0.04}^{-0.06}$ & $0.47_{+0.08}^{-0.06}$ & $0.17_{+0.02}^{-0.01}$ \\
\hline$\left|\Sigma_{c}^{04} P_{\lambda}{ }^{\frac{3}{2}}{ }^{-}\right\rangle \gamma$ & & $1.42_{+0.17}^{-0.16}$ & $0.55_{+0.04}^{-0.05}$ & $55.9_{+10.9}^{-8.6}$ & $84.22_{+16.3}^{-12.8}$ & $\begin{array}{l}44.2_{+9.2}^{-7.1} \\
\end{array}$ & $8.22_{+1.7}^{-1.3}$ \\
\hline$\left|\Sigma_{c}^{++4} P_{\lambda{ }^{\frac{5}{2}}}\right\rangle \gamma$ & 2789 & $2.66_{+0.48}^{-0.39}$ & $12.93_{+2.3}^{-1.8}$ & $10.1_{+2.7}^{-2.0}$ & $\begin{array}{l}32.3_{+6.5}^{-5.1} \\
\text {. }\end{array}$ & $49.4_{+9.5}^{-7.5}$ & $23.3_{+4.9}^{-3.8}$ \\
\hline $\mid \Sigma_{c}^{+4} P_{\lambda}$ & & $0.28_{+0.05}^{-0.04}$ & $1.36_{+0.25}^{-0.20}$ & $0.10_{+0.02}^{-0.02}$ & $0.42_{+0.06}^{-0.06}$ & $0.35_{+0.04}^{-0.03}$ & $0.55_{+0.11}^{-0.08}$ \\
\hline $\mid \Sigma_{c}^{04} P_{\lambda \frac{5}{2}}$ & & $0.33_{+0.05}^{-0.04}$ & $1.59_{+0.25}^{-0.20}$ & $6.72_{+.80}^{-1.28}$ & $19.6_{+4.2}^{-3.30}$ & $\begin{array}{l}35.0_{+7.3}^{-5.04} \\
\text {. }\end{array}$ & $30.5_{+6.9}^{-5.11}$ \\
\hline $\mid \Lambda_{c}^{+2} P_{\lambda} \frac{1}{2}$ & 2592 & $17.53_{+0.04}^{-0.02}$ & $25.36_{-2.2}^{-0.62}$ & $24.7_{+1.2}^{-1.7}$ & $88.8_{+2.3}^{-4.6}$ & $31.3_{+1.2}^{-2.2}$ & $0.35_{+0.24}^{-0.14}$ \\
\hline$\left|\Lambda_{c}^{+2} P_{\lambda \frac{3}{2}-}\right\rangle \gamma$ & 2628 & $64.6_{+5.0}^{-5.6}$ & $42.95_{+2.1}^{-3.0}$ & $21.2_{+2.2}^{-2.2}$ & $7.71_{+1.27}^{-1.07}$ & $53.9_{+2.5}^{-3.5}$ & $45.4_{+1.2}^{-2.4}$ \\
\hline & & $\left|\Sigma_{b}^{2} D_{\lambda \lambda}{ }^{\frac{3}{2}}{ }^{+}\right\rangle(6326)$ & $\left|\Sigma_{b}^{2} D_{\lambda \lambda \frac{5}{2}}{ }^{+}\right\rangle(6284)$ & $\left|\Sigma_{b}{ }^{4} D_{\lambda \lambda^{\frac{1}{2}}}{ }^{+}\right\rangle(6311)$ & $\left|\Sigma_{b}{ }^{4} D_{\lambda \lambda 2^{\frac{3}{2}}}{ }^{+}\right\rangle(6285)$ & $\left|\Sigma_{b}{ }^{4} D_{\lambda \lambda 2^{\frac{5}{2}}}{ }^{+}\right\rangle(6270)$ & $\left|\Sigma_{b}{ }^{4} D_{\lambda \lambda \overline{2}^{+}}{ }^{+}\right\rangle(6260)$ \\
\hline Decay mode & $M_{f}$ & $\Gamma_{i}(\mathrm{keV})$ & $\Gamma_{i}(\mathrm{keV})$ & $\Gamma_{i}(\mathrm{keV})$ & $\Gamma_{i}(\mathrm{keV})$ & $\Gamma_{i}(\mathrm{keV})$ & $\Gamma_{i}(\mathrm{keV})$ \\
\hline $\mid \Sigma_{b}^{+2} P_{\lambda \frac{1}{2}}$ & 6101 & $216.0_{+40.65}^{-32.4}$ & $15.90_{+3.42}^{-2.59}$ & $2.22_{+0.39}^{-0.31}$ & $2.79_{+0.50}^{-0.41}$ & $0.72_{+0.14}^{-0.11}$ & $<0.01$ \\
\hline $\mid \Sigma_{b}^{02} P_{\lambda} \frac{1}{2}$ & & $15.9_{+3.0}^{-2.40}$ & $1.09_{+0.24}^{-0.48}$ & $0.11_{+0.02}^{+0.01}$ & $0.17_{+0.03}^{+0.03}$ & 0.04 & $<0.01$ \\
\hline$\left|\Sigma_{b}^{-2} P_{\lambda \frac{1}{2}}{ }^{-}\right\rangle \gamma$ & & $45.3_{+8.4}^{-6.7}$ & $3.61_{+0.77}^{-0.58}$ & $0.67_{+0.12}^{-0.10}$ & $0.72_{+0.13}^{-0.11}$ & $0.19_{+0.03}^{-0.03}$ & $<0.01$ \\
\hline$\left|\Sigma_{b}^{+2} P_{\lambda \frac{3}{2}}{ }^{-}\right\rangle \gamma$ & 6096 & $141.0_{+28.2}^{-21.9}$ & $74.4_{+46.5}^{-12.5}$ & $1.50_{+0.28}^{-0.12}$ & $0.26_{+0.06}^{-0.04}$ & $2.04_{+0.36}^{-0.30}$ & $1.53_{+0.27}^{-0.22}$ \\
\hline 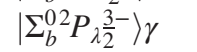 & & $9.84_{+1.98}^{-1.55}$ & $5.86_{+1.31}^{-0.99}$ & 0.09 & 0.02 & $0.12_{+0.03}^{-0.01}$ & 0.09 \\
\hline$\left|\Sigma_{b}^{-2} P_{\lambda \frac{3}{2}}^{-}\right\rangle \gamma$ & & $31.4_{+6.2}^{+4.98}$ & $14.3_{+3.18}^{-2.36}$ & $0.39_{+0.07}^{-0.06}$ & 0.06 & $0.52_{+0.10}^{-0.07}$ & $0.39_{+0.07}^{-0.05}$ \\
\hline$\left|\Sigma_{b}^{+4} P_{\lambda \frac{1}{2}}{ }^{-}\right\rangle \gamma$ & 6095 & $4.92_{+0.49}^{-0.50}$ & $0.13_{+0.02}^{+0.18}$ & $243.8_{+44.7}^{-35.9}$ & $67.54_{+13.6}^{-10.5}$ & $\begin{array}{l}9.69_{+2.05}^{-1.58} \\
\end{array}$ & $1.64_{+0.48}^{-0.34}$ \\
\hline$\left|\Sigma_{b}^{04} P_{\lambda \frac{1}{2}}\right\rangle \gamma$ & & $0.30_{+0.03}^{-0.03}$ & 0.008 & $17.6_{+3.3}^{-2.6}$ & $4.94_{+1.00}^{-0.78}$ & $0.68_{+0.15}^{-0.11}$ & $0.12_{+0.04}^{-0.02}$ \\
\hline$\left|\Sigma_{b}^{-4} P_{\lambda \frac{1}{2}}{ }^{-}\right\rangle \gamma$ & & $1.27_{+0.13}^{-0.13}$ & 0.034 & $52.1_{+9.4}^{-7.5}$ & $14.2_{+2.9}^{-2.2}$ & $2.14_{+0.45}^{-0.15}$ & $0.34_{+0.10}^{-0.07}$ \\
\hline$\left|\Sigma_{b}^{+4} P_{\lambda \frac{3}{2}}^{-}\right\rangle \gamma$ & 6087 & $9.18_{+1.25}^{-1.11}$ & $1.74_{+0.25}^{-0.21}$ & $124.8_{+24.0}^{-19.0}$ & $124.9_{+24.9}^{-19.4}$ & $62.7_{+13.3}^{+0.43}$ & $11.0_{+2.3}^{-1.8}$ \\
\hline$\left|\Sigma_{b}^{04} P_{\lambda \lambda^{\frac{3}{2}}}\right\rangle \gamma$ & & $0.56_{+0.07}^{-0.07}$ & $0.11_{+0.01}^{-0.02}$ & $8.78_{+1.71}^{-1.34}$ & $9.05_{+1.82}^{-1.42}$ & $4.75_{+1.02}^{-0.77}$ & $0.78_{+0.16}^{-0.13}$ \\
\hline$\left|\Sigma_{b}^{-4} P_{\lambda \frac{3}{2}}{ }^{-}\right\rangle \gamma$ & & $2.37_{+0.32}^{-0.29}$ & $0.45_{+0.06}^{-0.06}$ & $27.5_{+5.2}^{-4.1}$ & $26.6_{+5.3}^{-4.1}$ & $12.7_{+2.7}^{-2.1}$ & $2.39_{+0.51}^{-0.39}$ \\
\hline$\left|\Sigma_{b}^{+4} P_{\lambda \frac{5}{2}}\right\rangle \gamma$ & 6084 & $2.88_{+0.48}^{-0.39}$ & $6.53_{+1.15}^{-0.94}$ & $19.9_{+5.8}^{-4.0}$ & $37.4_{+8.3}^{-6.3}$ & $62.8_{+13.3}^{-10.2}$ & $50.4_{+11.4}^{-8.6}$ \\
\hline$\left|\Sigma_{b}^{04} P_{\lambda{ }^{2}}{ }^{-}\right\rangle \gamma$ & & $0.17_{+0.03}^{-0.02}$ & $0.40_{+0.06}^{-0.07}$ & $1.43_{+0.40}^{-0.29}$ & $2.65_{+0.59}^{-0.45}$ & $4.57_{+0.97}^{-0.75}$ & $4.01_{+0.91}^{-0.68}$ \\
\hline$\left|\Sigma_{b}^{-4} P_{\lambda^{\frac{5}{2}}}\right\rangle \gamma$ & & $0.74_{+0.13}^{-0.10}$ & $1.68_{+0.30}^{-0.24}$ & $4.31_{+1.25}^{-0.87}$ & $8.18_{+1.80}^{-1.36}$ & $13.3_{+2.8}^{-2.1}$ & $9.59_{+2.15}^{-1.62}$ \\
\hline$\left|\Lambda_{b}^{02} P_{\lambda \frac{1}{2}-}\right\rangle \gamma$ & 5912 & $19.0_{-2.1}^{+0.9}$ & $21.6_{-2.2}^{+0.7}$ & $27.6_{+0.8}^{-1.6}$ & $79.0_{+2.3}^{-4.3}$ & $27.4_{+1.6}^{-2.0}$ & $0.35_{+0.25}^{-0.14}$ \\
\hline$\left|\Lambda_{b}^{02} P_{\lambda^{\frac{3}{2}}}{ }^{-}\right\rangle \gamma$ & 5920 & $99.0_{+3.7}^{-5.9}$ & $45.8_{+1.6}^{-2.6}$ & $30.2_{+2.5}^{-2.7}$ & $9.06_{+1.56}^{-1.28}$ & $56.1_{+1.5}^{-3.0}$ & $45.4_{+0.03}^{-1.7}$ \\
\hline
\end{tabular}

\section{2. $J^{P}=3 / 2^{+}$states}

The $J^{P}=3 / 2^{+}$state $\left|\Sigma_{c}{ }^{2} D_{\lambda \lambda 2^{\frac{3}{2}}}{ }^{+}\right\rangle$dominantly decays into the $P$-wave states through the pionic decay modes $\Lambda_{c}(2625) \pi$ and $\left|\Sigma_{c}{ }^{2} P_{\lambda{ }^{3}}-\right\rangle \pi$, while its decay rate into $\Sigma_{c} \pi$ is sizable. It has a width of $\mathcal{O}(10)-\mathcal{O}(100) \mathrm{MeV}$, which significantly depends on its mass. If the mass is taken as the prediction $3043 \mathrm{MeV}$ in Ref. [21], the sum of the partial widths of the pionic decays is $\Gamma_{\text {Sum }} \sim 100 \mathrm{MeV}$ (see Table VIII), and the ratios between the partial decay widths for the $\Lambda_{c}(2625) \pi$ and $\Sigma_{c} \pi$ channels and $\Gamma_{\text {Sum }}$ are predicted to be 


$$
\frac{\Gamma\left[\Lambda_{c}(2625) \pi\right]}{\Gamma_{\text {Sum }}} \simeq 50 \%, \quad \frac{\Gamma\left[\Sigma_{c} \pi\right]}{\Gamma_{\text {Sum }}} \simeq 7 \% .
$$

The $\Sigma_{c} \pi$ and $\Lambda_{c}(2625) \pi$ decay channels may be ideal channels for our search for $\left|\Sigma_{c}{ }^{2} D_{\lambda \lambda^{2}}{ }^{+}\right\rangle$in future experiments.

For the other $J^{P}=3 / 2^{+}$state $\left|\Sigma_{c}{ }^{4} D_{\lambda \lambda^{2}}{ }^{+}\right\rangle$, one finds that it has large decay rates into the $P$-wave states through the pionic decay modes $\Lambda_{c}(2625) \pi,\left|\Sigma_{c}{ }^{4} P_{\lambda{ }^{2}}{ }^{-}\right\rangle \pi,\left|\Sigma_{c}{ }^{4} P_{\lambda^{2}}{ }^{-}\right\rangle \pi$, and $\left|\Sigma_{c}{ }^{4} P_{\lambda \frac{2}{2}^{-}}\right\rangle \pi$. Its width should be broader than that of $\left|\Sigma_{c}^{2} D_{\lambda \lambda 2^{3}}{ }^{+}\right\rangle$. Furthermore, the decay rate into $\Sigma_{c}(2520) \pi$ is sizable as well. If its mass is taken as the prediction $3040 \mathrm{MeV}$ in Ref. [21], the sum of the partial widths of the pionic decays can reach up to $\Gamma_{\text {Sum }} \sim 120 \mathrm{MeV}$ (see Table VIII), while the ratios between the partial widths of $\Lambda_{c}(2625) \pi$ and $\Sigma_{c}(2520) \pi$ and $\Gamma_{\text {Sum }}$ are predicted to be

$$
\frac{\Gamma\left[\Lambda_{c}(2625) \pi\right]}{\Gamma_{\text {Sum }}} \simeq 35 \%, \quad \frac{\Gamma\left[\Sigma_{c}(2520) \pi\right]}{\Gamma_{\text {Sum }}} \simeq 6 \% .
$$

The $\Lambda_{c}(2625) \pi$ and $\Sigma_{c}(2520) \pi$ may be ideal channels for our search for $\left|\Sigma_{c}^{4} D_{\lambda \lambda^{2}}{ }^{+}\right\rangle$in future experiments.

We also estimate the radiative transitions of these $J^{P}=$ $3 / 2^{+}$states into the $1 P$-wave states. Our results are listed in Table IX. It is found that $\left|\Sigma_{c}^{++(0)}{ }^{4} D_{\lambda \lambda^{3}}{ }^{+}\right\rangle$might have relatively large decay rates into $\left|\Sigma_{c}^{++(0)}{ }^{4} P_{\lambda^{2}}{ }^{3}\right\rangle$, while $\left|\Sigma_{c}^{+4} D_{\lambda \lambda \frac{3}{2}}{ }^{+}\right\rangle$might have a relatively large decay rates into $\Lambda_{c}(2595)^{+} \gamma$, and their partial radiative decay widths are estimated to be $\mathcal{O}(10)-\mathcal{O}(100) \mathrm{keV}$. The branching fractions for these main radiative decay processes may reach up to $\mathcal{O}\left(10^{-4}\right)-\mathcal{O}\left(10^{-3}\right)$. The $\left|\Sigma_{c}^{+4} D_{\lambda \lambda^{\frac{3}{2}}}{ }^{+}\right\rangle$may have the possibility of being observed in the $\Lambda_{c}(2595)^{+} \gamma$ channel.

\section{3. $J^{P}=5 / 2^{+}$states}

The $J^{P}=5 / 2^{+}$state $\left|\Sigma_{c}{ }^{2} D_{\lambda \lambda 2^{2}}{ }^{+}\right\rangle$might be a narrow state with a width of $\mathcal{O}(10) \mathrm{MeV}$ (see Fig. 3). It has large decay rates into $\Lambda_{c} \pi, \Sigma_{c} \pi, \Lambda_{c}(2595) \pi$, and $\Lambda_{c}(2625) \pi$ with comparable partial decay widths. If its mass is taken as the prediction $3038 \mathrm{MeV}$ in Ref. [21], the sum of the partial widths of the pionic decays is about $\Gamma_{\text {Sum }} \sim 60 \mathrm{MeV}$ (see Table VIII), and the ratios between the partial widths for the main decay modes, $\Lambda_{c} \pi, \Sigma_{c} \pi, \Lambda_{c}(2595) \pi$, and $\Lambda_{c}(2625) \pi$, and $\Gamma_{\text {Sum }}$ are predicted to be

$$
\begin{aligned}
& \frac{\Gamma\left[\Lambda_{c} \pi, \Sigma_{c} \pi, \Lambda_{c}(2595) \pi, \Lambda_{c}(2625) \pi\right]}{\Gamma_{\text {Sum }}} \\
& \quad \simeq 20 \%, 15 \%, 18 \%, 19 \% .
\end{aligned}
$$

The $\Lambda_{c} \pi, \Sigma_{c} \pi, \Lambda_{c}(2595) \pi$, and $\Lambda_{c}(2625) \pi$ decay channels may be ideal channels for our search for the missing $J^{P}=$ $5 / 2^{+}$state $\left|\Sigma_{c}{ }^{2} D_{\lambda \lambda \frac{5}{2}}{ }^{+}\right\rangle$.

The other $J^{P}=5 / 2^{+}$state $\left|\Sigma_{c}{ }^{4} D_{\lambda \lambda^{2}}{ }^{+}\right\rangle$might also be a narrow state with a width of $\mathcal{O}(10) \mathrm{MeV}$. This state has relatively large decay rates into $\Sigma_{c}(2520) \pi, \Lambda_{c}(2595) \pi$, $\left|\Sigma_{c}{ }^{4} P_{\lambda} \frac{1}{2}^{-}\right\rangle \pi$, and $\left|\Sigma_{c}{ }^{4} P_{\lambda^{2}}{ }^{-}\right\rangle \pi$. If its mass is taken as the prediction $3023 \mathrm{MeV}$ in Ref. [21], the sum of the partial widths of the pionic decays is about $\Gamma_{\text {Sum }} \sim 70 \mathrm{MeV}$ (see Table VIII), while the ratios between the partial widths for the main decay modes, $\Sigma_{c}(2520) \pi$ and $\Lambda_{c}(2595) \pi$, and $\Gamma_{\text {Sum }}$ are predicted to be

$$
\frac{\Gamma\left[\Sigma_{c}(2520) \pi, \Lambda_{c}(2595) \pi\right]}{\Gamma_{\text {Sum }}} \simeq 17 \%, 16 \% .
$$

The $\Sigma_{c}(2520) \pi$ and $\Lambda_{c}(2595) \pi$ may be ideal channels for a search for $\left|\Sigma_{c}{ }^{4} D_{\lambda \lambda^{2}}{ }^{+}\right\rangle$in future experiments.

The radiative transitions of these $J^{P}=5 / 2^{+}$states into the $1 P$-wave charmed baryon states are estimated as well. Our results are listed in Table IX. It is found that for the $\left|\Sigma_{c}{ }^{2} D_{\lambda \lambda^{2}}{ }^{+}\right\rangle$state the main radiative decay processes are $\left|\Sigma_{c}^{++(0) 2} D_{\lambda \lambda} \lambda^{\frac{5}{2}}\right\rangle \rightarrow\left|\Sigma_{c}^{++(0)}{ }^{2} P_{\lambda} \frac{1}{2}^{-}\right\rangle \gamma, \quad\left|\Sigma_{c}^{++(0)}{ }^{2} P_{\lambda \frac{3}{2}}{ }^{-}\right\rangle \gamma, \quad$ and $\left|\Sigma_{c}^{+2} D_{\lambda \lambda^{\frac{5}{2}}}{ }^{+}\right\rangle \rightarrow \Lambda_{c}(2595,2625)^{+} \gamma$, while for the other $J^{P}=$ $5 / 2^{+}$state $\left|\Sigma_{c}{ }^{4} D_{\lambda \lambda^{2}}{ }^{+}\right\rangle$, the main radiative decay processes are $\left|\Sigma_{c}^{++(0)}{ }_{4} D_{\lambda \lambda^{\frac{5}{2}}}{ }^{+}\right\rangle \rightarrow\left|\Sigma_{c}^{++(0)}{ }^{4} P_{\lambda^{\frac{3}{2}}}{ }^{-}\right\rangle \gamma,\left|\Sigma_{c}^{++(0)}{ }^{4} P_{\lambda \frac{5}{2}}\right\rangle \gamma$, and $\left|\Sigma_{c}^{+4} D_{\lambda \lambda \frac{5}{2}}{ }^{+}\right\rangle \rightarrow \Lambda_{c}(2595,2625)^{+} \gamma$. The partial radiative decay widths for these processes are estimated to be $\mathcal{O}(10) \mathrm{keV}$, while the branching fractions may be $\mathcal{O}\left(10^{-4}\right)$. These radiative transitions may be hard to be observed in experiments.

\section{4. $J^{P}=7 / 2^{+}$state}

The $J^{P}=7 / 2^{+}$state $\left|\Sigma_{c}^{4} D_{\lambda \lambda \lambda^{\frac{7}{2}}}\right\rangle$ might have a relatively narrow width of $\mathcal{O}(10) \mathrm{MeV}$. It mainly decays into $\Lambda_{c} \pi$, $\Lambda_{c}(2595) \pi$, and $\Lambda_{c}(2625) \pi$ channels. If one adopts the predicted mass $3013 \mathrm{MeV}$ in Ref. [21], the sum of the partial widths of the pionic decays is estimated to be $\Gamma_{\text {Sum }} \sim$ $60 \mathrm{MeV}$ (see Table VIII), and the ratios between the partial decay widths for these main channels, $\Lambda_{c} \pi, \Lambda_{c}(2595) \pi$, and $\Lambda_{c}(2625) \pi$, and $\Gamma_{\text {Sum }}$ are predicted to be

$\frac{\Gamma\left[\Lambda_{c} \pi, \Lambda_{c}(2595) \pi, \Lambda_{c}(2625) \pi\right]}{\Gamma_{\text {Sum }}} \simeq 22 \%, 19 \%, 41 \%$.

The $\Lambda_{c} \pi, \Lambda_{c}(2595) \pi$, and $\Lambda_{c}(2625) \pi$ may be ideal channels for a search for $\left|\Sigma_{c}{ }^{4} D_{\lambda \lambda} \lambda^{\frac{7}{2}}\right\rangle$ in future experiments.

The radiative transitions into the $1 P$-wave states are estimated as well. Our results are listed in Table IX. It is found that the main radiative decay processes are $\left|\Sigma_{c}^{++(0)}{ }^{4} D_{\lambda \lambda} \overline{7}^{+}\right\rangle \rightarrow\left|\Sigma_{c}^{++(0)}{ }^{4} P_{\lambda \frac{5}{2}}{ }^{-}\right\rangle \gamma \quad$ and $\quad\left|\Sigma_{c}^{+4} D_{\lambda \lambda} \lambda^{\frac{7}{2}}\right\rangle \rightarrow$ $\Lambda_{c}(2595)^{+} \gamma$. Their partial radiative decay widths are estimated to be $\mathcal{O}(10) \mathrm{keV}$, while the branching fractions are $\mathcal{O}\left(10^{-4}\right)$. These radiative transitions may be hard to be observed in experiments. 

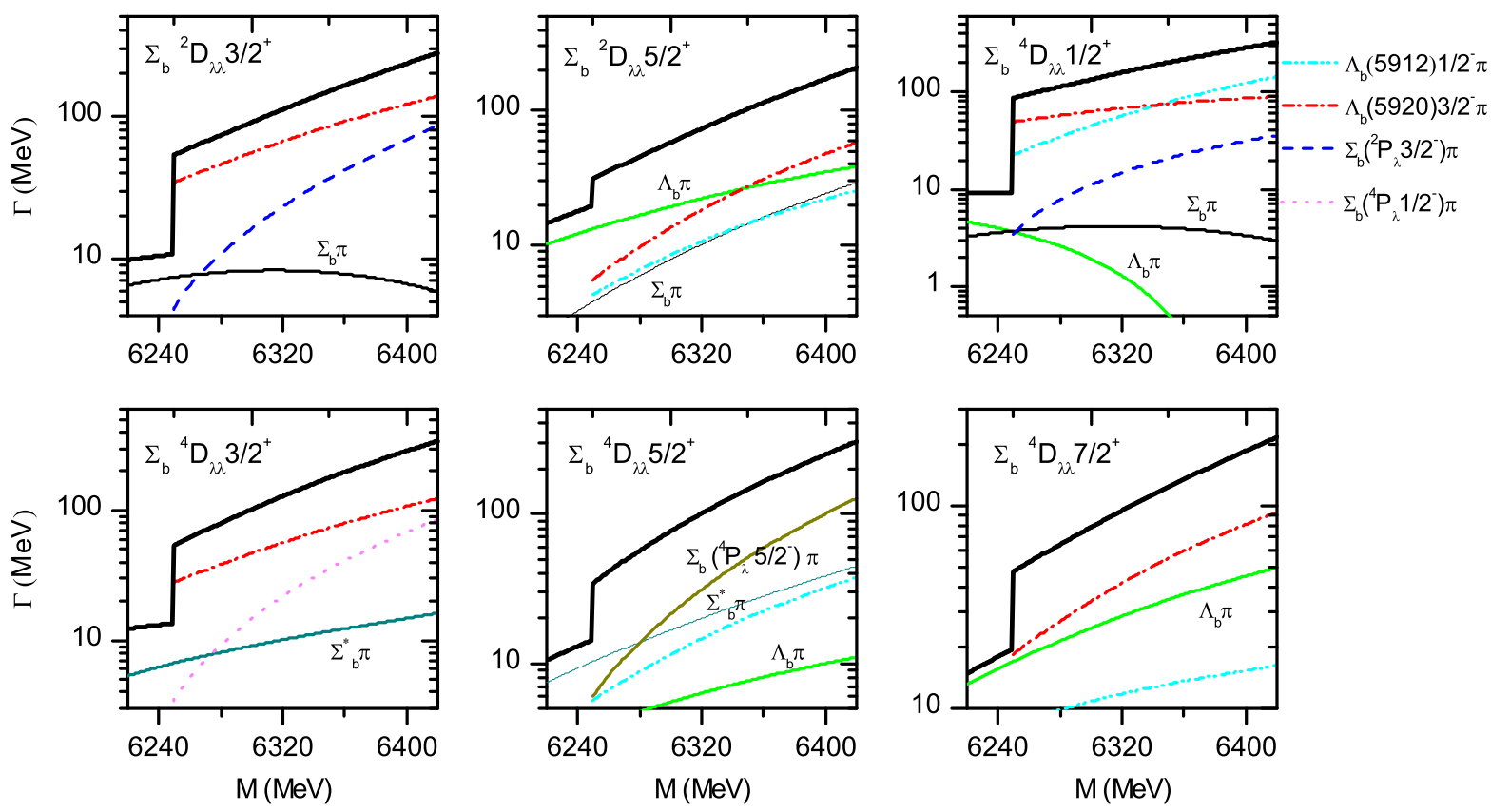

FIG. 4. Strong decay partial widths of the main decay modes for the $\lambda$-mode $1 D$-wave excited $\Sigma_{b}$ states as functions of their mass. The bold solid curves stand for the sum of the partial widths. The masses of the $P$-wave heavy baryons in final states are adopted from the quark model predictions in Ref. [21], if there are no observations (see Table II).

\section{B. $\Sigma_{b}$}

In the $\Sigma_{b}$ family, according to the quark model classification, there are six $\lambda$-mode $1 D$-wave excitations: $\left|\Sigma_{b}^{4} D_{\lambda \lambda^{\frac{1}{2}}}{ }^{+}\right\rangle, \quad\left|\Sigma_{b}^{4} D_{\lambda \lambda^{3}}{ }^{+}\right\rangle, \quad\left|\Sigma_{b}^{2} D_{\lambda \lambda^{\frac{3}{2}}}{ }^{+}\right\rangle, \quad\left|\Sigma_{b}^{2} D_{\lambda \lambda^{\frac{5}{2}}}{ }^{+}\right\rangle$, $\left|\Sigma_{b}{ }^{4} D_{\lambda \lambda^{\frac{5}{2}}}{ }^{+}\right\rangle$, and $\left|\Sigma_{b}{ }^{4} D_{\lambda \lambda^{2}}{ }^{+}\right\rangle$. However, no $1 D$-wave states have been established. The typical masses of the $\lambda$-mode $1 D$-wave $\Sigma_{b}$ excitations are predicted to be $\sim 6.3$ within various quark models (see Table II). In the possible mass ranges, we study their strong decay transitions within ChQM. Our results are shown in Fig. 4. To be more specific, taking the masses of the $1 D$-wave states obtained in the relativistic quark-diquark picture [21], we present the results in Table VIII.

\section{1. $J^{P}=1 / 2^{+}$state}

The $J^{P}=1 / 2^{+}$state $\left|\Sigma_{b}{ }^{4} D_{\lambda \lambda}{ }^{1}{ }^{+}\right\rangle$might be a broad state with a width of $\mathcal{O}(100) \mathrm{MeV}$. If its mass is taken as the prediction in Ref. [21], the sum of the partial widths for the pionic decays can reach up to $\Gamma_{\text {Sum }} \sim 150 \mathrm{MeV}$ (see Table VIII). This state might mainly decay into the $P$-wave $\Lambda_{b}$ states $\Lambda_{b}(5912) \frac{1}{2}^{-}$and $\Lambda_{b}(5920) \frac{3}{2}-$ via pionic decay modes $\Lambda_{b}(5912) \pi$ and $\Lambda_{b}(5920) \pi$. The ratios between the partial decay widths for the $\Lambda_{b}(5912) \pi$ and $\Lambda_{b}(5920) \pi$ channels and $\Gamma_{\text {Sum }}$ are predicted to be

$$
\frac{\Gamma\left[\Lambda_{b}(5912) \pi\right]}{\Gamma_{\text {Sum }}} \simeq 35 \%, \quad \frac{\Gamma\left[\Lambda_{b}(5920) \pi\right]}{\Gamma_{\text {Sum }}} \simeq 44 \%
$$

Both $\Lambda_{b}(5912) \pi$ and $\Lambda_{b}(5920) \pi$ may be ideal channels for our search for $\left|\Sigma_{b}{ }^{4} D_{\lambda \lambda^{2}}{ }^{+}\right\rangle$in future experiments.

We also estimate its radiative transitions. Our results are listed in Table IX. It is found that $\left|\Sigma_{b}^{+4} D_{\lambda \lambda^{2}}{ }^{+}\right\rangle$might have relatively large decay rates into $\left|\Sigma_{b}^{+4} P_{\lambda \frac{1}{2}}{ }^{-}\right\rangle \gamma$ and $\left|\Sigma_{b}^{+4} P_{\lambda \frac{3}{2}}{ }^{-}\right\rangle \gamma$, and their partial radiative decay widths are estimated to be $\mathcal{O}(100) \mathrm{keV}$. The branching fractions for these main radiative decay processes may reach up to $\mathcal{O}\left(10^{-3}\right)$.

\section{2. $J^{P}=3 / 2^{+}$states}

The $J^{P}=3 / 2^{+}$state $\left|\Sigma_{b}{ }^{2} D_{\lambda \lambda^{2}}{ }^{+}\right\rangle$has large decay rates into the $P$-wave states through the pionic decay modes $\Lambda_{b}(5920) \frac{3}{2}^{-} \pi$ and $\left|\Sigma_{b}{ }^{2} P_{\lambda 2^{\frac{3}{2}}}{ }^{-}\right\rangle$. It has a width of $\mathcal{O}(10)-$ $\mathcal{O}(100) \mathrm{MeV}$, which obviously depends on its mass (see Fig. 4). If the mass is taken as the prediction $6326 \mathrm{MeV}$ in Ref. [21], the sum of the partial widths of the pionic decays can reach up to $\Gamma_{\text {Sum }} \sim 120 \mathrm{MeV}$ (see Table VIII), and the ratio between the partial decay width for the $\Lambda_{b}(5920) \pi$ channel and $\Gamma_{\text {Sum }}$ is predicted to be

$$
\frac{\Gamma\left[\Lambda_{b}(5920) \pi\right]}{\Gamma_{\text {Sum }}} \simeq 58 \% .
$$

The $\Lambda_{b}(5920) \pi$ decay channel may be an ideal channel for our search for $\left|\Sigma_{b}^{2} D_{\lambda \lambda^{3}}{ }^{+}\right\rangle$in future experiments.

For the other $J^{P}=3 / 2^{+}$state $\left|\Sigma_{b}{ }^{4} D_{\lambda \lambda^{2}}{ }^{+}\right\rangle$, one finds that this state mainly decays into the $P$-wave states through the pionic decay modes $\Lambda_{b}(5920) \pi,\left|\Sigma_{b}{ }^{4} P_{\lambda \frac{1}{2}}-\right\rangle \pi$, and $\left|\Sigma_{b}{ }^{4} P_{\lambda{ }^{5}}{ }^{-}\right\rangle \pi$. Furthermore, the decay rate into $\Sigma_{b}^{*}(5832) \pi$ 
is sizable as well. Its width should be narrower than that of $\left|\Sigma_{b}^{2} D_{\lambda \lambda^{2}}{ }^{+}\right\rangle$. If its mass is taken as the predictions in Ref. [21], the sum of the partial widths of the pionic decays can reach up to $\Gamma_{\text {Sum }} \sim 85 \mathrm{MeV}$ (see Table VIII), while the ratios between the partial widths of $\Gamma\left[\Sigma_{b}^{*}(5832) \pi\right]$ and $\Gamma\left[\Lambda_{b}(5920) \pi\right]$ and $\Gamma_{\text {Sum }}$ are predicted to be

$\frac{\Gamma\left[\Sigma_{b}^{*}(5832) \pi\right]}{\Gamma_{\text {Sum }}} \simeq 10 \%, \quad \frac{\Gamma\left[\Lambda_{b}(5920) \pi\right]}{\Gamma_{\text {Sum }}} \simeq 46 \%$.

Both $\Sigma_{b}^{*}(5832) \pi$ and $\Lambda_{b}(5920) \pi$ may be ideal channels for a search for $\left|\Sigma_{b}^{4} D_{\lambda \lambda^{2}}{ }^{+}\right\rangle$in future experiments.

The radiative decays of the $J^{P}=3 / 2^{+}$states into the $1 P$ wave bottom baryon states are estimated as well. Our results are listed in Table IX. It is found that $\left|\Sigma_{b}^{+2} D_{\lambda \lambda^{2}}{ }^{+}\right\rangle$ might have relatively large decay rates into $\left|\Sigma_{b}^{+2} P_{\lambda} \frac{1}{2}{ }^{-}\right\rangle \gamma$ and $\left|\Sigma_{b}^{+2} P_{\lambda{ }^{\frac{3}{2}}}{ }^{-}\right\rangle \gamma$, while $\left|\Sigma_{b}^{+4} D_{\lambda \lambda^{3}}{ }^{+}\right\rangle$might have a relatively large decay rate into $\left|\Sigma_{b}^{+4} P_{\lambda \frac{3}{2}}{ }^{-}\right\rangle \gamma$. Their partial radiative decay widths are estimated to be $\mathcal{O}(100) \mathrm{keV}$, while the branching fractions may reach up to $\mathcal{O}\left(10^{-3}\right)$.

\section{3. $J^{P}=5 / 2^{+}$states}

The $\left|\Sigma_{b}^{2} D_{\lambda \lambda^{2}}{ }^{+}\right\rangle$might be a relatively narrow state with a width of $\mathcal{O}(10) \mathrm{MeV}$. It has large decay rates into $\Lambda_{b} \pi$, $\Sigma_{b} \pi, \Lambda_{b}(5912) \pi$, and $\Lambda_{b}(5920) \pi$. If its mass is taken as the prediction $6284 \mathrm{MeV}$ in Ref. [21], the sum of the partial widths of the pionic decays is about $\Gamma_{\text {Sum }} \sim 50 \mathrm{MeV}$ (see Table VIII), and the ratios between the partial decay widths for these main channels, $\Lambda_{b} \pi, \Sigma_{b} \pi, \Lambda_{b}(5912) \pi$, and $\Lambda_{b}(5920) \pi$, and $\Gamma_{\text {Sum }}$ are predicted to be

$$
\begin{gathered}
\frac{\Gamma\left[\Lambda_{b} \pi, \Sigma_{b} \pi, \Lambda_{b}(5912) \pi, \Lambda_{b}(5920) \pi\right]}{\Gamma_{\text {Sum }}} \\
\quad \simeq 35 \%, 13 \%, 14 \%, 21 \% .
\end{gathered}
$$

The $\Lambda_{b} \pi, \Sigma_{b} \pi, \Lambda_{b}(5912) \pi$, and $\Lambda_{b}(5920) \pi$ decay channels may be ideal channels for our search for $\left|\Sigma_{b}^{2} D_{\lambda \lambda \frac{5}{2}}{ }^{+}\right\rangle$in future experiments.

For the other $J^{P}=5 / 2^{+}$state $\left|\Sigma_{b}{ }^{4} D_{\lambda \lambda \frac{5}{2}}{ }^{+}\right\rangle$, it has a width of $\mathcal{O}(10)-\mathcal{O}(100) \mathrm{MeV}$, which significantly depends on the mass. This state has large decay rates into $\Lambda_{b} \pi, \Sigma_{b}^{*} \pi$, $\Lambda_{b}(5912) \pi,\left|\Sigma_{b}{ }^{4} P_{\lambda \frac{1}{2}}-\right\rangle \pi$, and $\left|\Sigma_{b}{ }^{4} P_{\lambda \frac{5}{2}}-\right\rangle \pi$. If its mass is taken as the prediction $6270 \mathrm{MeV}$ in Ref. [21], the sum of the partial widths of the pionic decays is about $\Gamma_{\text {Sum }} \sim$ $50 \mathrm{MeV}$ (see Table VIII), and the ratios between the partial decay widths for the $\Lambda_{b} \pi, \Sigma_{b}^{*} \pi$, and $\Lambda_{b}(5912) \pi$ final states and $\Gamma_{\text {Sum }}$ are predicted to be

$$
\frac{\Gamma\left[\Lambda_{b} \pi, \Sigma_{b}^{*} \pi, \Lambda_{b}(5912) \pi\right]}{\Gamma_{\text {Sum }}} \simeq 9 \%, 26 \%, 16 \% .
$$

The $\Lambda_{b} \pi, \Sigma_{b}^{*} \pi$, and $\Lambda_{b}(5912) \pi$ decay channels might be ideal channels for our search for $\left|\Sigma_{b}{ }^{4} D_{\lambda \lambda \frac{5}{2}}{ }^{+}\right\rangle$in future experiments.
The radiative decays of these $J^{P}=5 / 2^{+}$states into the $1 P$-wave bottom baryon states are estimated as well. Our results are listed in Table IX. It is found that for the $\left|\Sigma_{b}{ }^{2} D_{\lambda \lambda 2^{2}}{ }^{+}\right\rangle$state the main radiative decay processes are $\left|\Sigma_{b}^{+2} D_{\lambda \lambda 2^{\frac{5}{2}}}\right\rangle \rightarrow\left|\Sigma_{b}^{+2} P_{\lambda \frac{1}{2}^{-}}\right\rangle \gamma,\left|\Sigma_{b}^{+2} P_{\lambda{ }^{\frac{1}{2}}}{ }^{-}\right\rangle \gamma$, and $\left|\Sigma_{b}^{02} D_{\lambda \lambda^{\frac{5}{2}}}{ }^{+}\right\rangle \rightarrow$ $\Lambda_{b}(5912,5920)^{+} \gamma$, while for the other $J^{P}=5 / 2^{+}$ state $\left|\Sigma_{b}{ }^{4} D_{\lambda \lambda^{2}}{ }^{+}\right\rangle$, the main radiative decay processes are $\quad\left|\Sigma_{b}^{+4} D_{\lambda \lambda \lambda^{2}}{ }^{-}\right\rangle \rightarrow\left|\Sigma_{b}^{+4} P_{\lambda^{2}}{ }^{-}\right\rangle \gamma, \quad\left|\Sigma_{b}^{+4} P_{\lambda \frac{5}{2}}^{-}\right\rangle \gamma, \quad$ and $\left|\Sigma_{b}^{04} D_{\lambda \lambda^{\frac{5}{2}}}\right\rangle \rightarrow \Lambda_{b}(5912,5920)^{+} \gamma$. The partial radiative decay widths for these processes are estimated to be $\mathcal{O}(10) \mathrm{keV}$, while the branching fractions may be $\mathcal{O}\left(10^{-4}\right)-\mathcal{O}\left(10^{-3}\right)$.

$$
\text { 4. } J^{P}=7 / 2^{+} \text {state }
$$

The $J^{P}=7 / 2^{+}$state $\left|\Sigma_{b}{ }^{4} D_{\lambda \lambda^{2}}{ }^{+}\right\rangle$might have a width of $\mathcal{O}(10)-\mathcal{O}(100) \mathrm{MeV}$, which strongly depends on its mass (see Fig. 4). This state has large decay rates into $\Lambda_{b} \pi$, $\Lambda_{b}(5912) \pi$, and $\Lambda_{b}(5920) \pi$ channels. If one adopts the predicted mass $6260 \mathrm{MeV}$ in Ref. [21], the sum of the partial widths of the pionic decays is estimated to be $\Gamma_{\text {Sum }} \sim$ $50 \mathrm{MeV}$ (see Table VIII), and the ratios between the partial decay widths for these main channels, $\Lambda_{b} \pi, \Lambda_{b}(5912) \pi$, and $\Lambda_{b}(5920) \pi$, and $\Gamma_{\text {Sum }}$ are predicted to be

$\frac{\Gamma\left[\Lambda_{b} \pi, \Lambda_{b}(5912) \pi, \Lambda_{b}(5920) \pi\right]}{\Gamma_{\mathrm{Sum}}} \simeq 35 \%, 16 \%, 40 \%$.

The $\Lambda_{b} \pi, \Lambda_{b}(5912) \pi$, and $\Lambda_{b}(5920) \pi$ decay channels may be ideal channels for our search for $\left|\Sigma_{b}{ }^{4} D_{\lambda \lambda^{2}}{ }^{7}\right\rangle$ in future experiments.

We also estimate its radiative decays into the $1 P$-wave bottom baryon states. Our results are listed in Table IX. It is found that the main radiative decay processes are $\left|\Sigma_{b}^{+4} D_{\lambda \lambda^{\frac{7}{2}}}\right\rangle \rightarrow\left|\Sigma_{b}^{+4} P_{\lambda{ }^{2}}{ }^{-}\right\rangle \gamma$ and $\left|\Sigma_{b}^{04} D_{\lambda \lambda^{\frac{7}{2}}}{ }^{+}\right\rangle \rightarrow \Lambda_{b}(5920)^{0} \gamma$. Their partial radiative decay widths are estimated to be $\mathcal{O}(10) \mathrm{keV}$, while the branching fractions may be $\mathcal{O}\left(10^{-4}\right)-\mathcal{O}\left(10^{-3}\right)$. The neutral $J^{P}=7 / 2^{+}$state $\left|\Sigma_{b}^{04} D_{\lambda \lambda \frac{7}{2}}{ }^{+}\right\rangle$may have the possibility of being observed in the $\Lambda_{b}(5920)^{0} \gamma$ channel.

\section{C. $\boldsymbol{\Xi}_{c}^{\prime}$}

In the $\Xi_{c}^{\prime}$ family, according to the quark model classification, there are six $\lambda$-mode $1 D$-wave excitations:

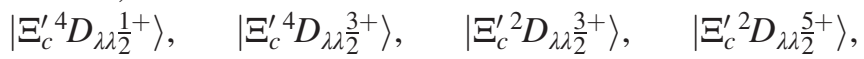

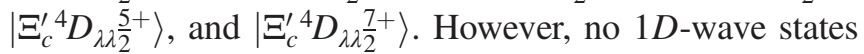
have been established. The typical masses of the $\lambda$-mode $1 D$-wave $\Xi_{c}^{\prime}$ excitations are $\sim 3.14$ within various quark model predictions (see Table II).

In Ref. [48], the strong decay properties of the $D$-wave excited $\Xi_{c}^{\prime}$ states were studied in their possible mass ranges. However, we did not give correct predictions of the partial widths of the $D$-wave excited $\Xi_{c}^{\prime}$ states decaying into the $P$-wave charmed baryons. In this work, we update our predictions, which have been shown in Fig. 5. To be more 

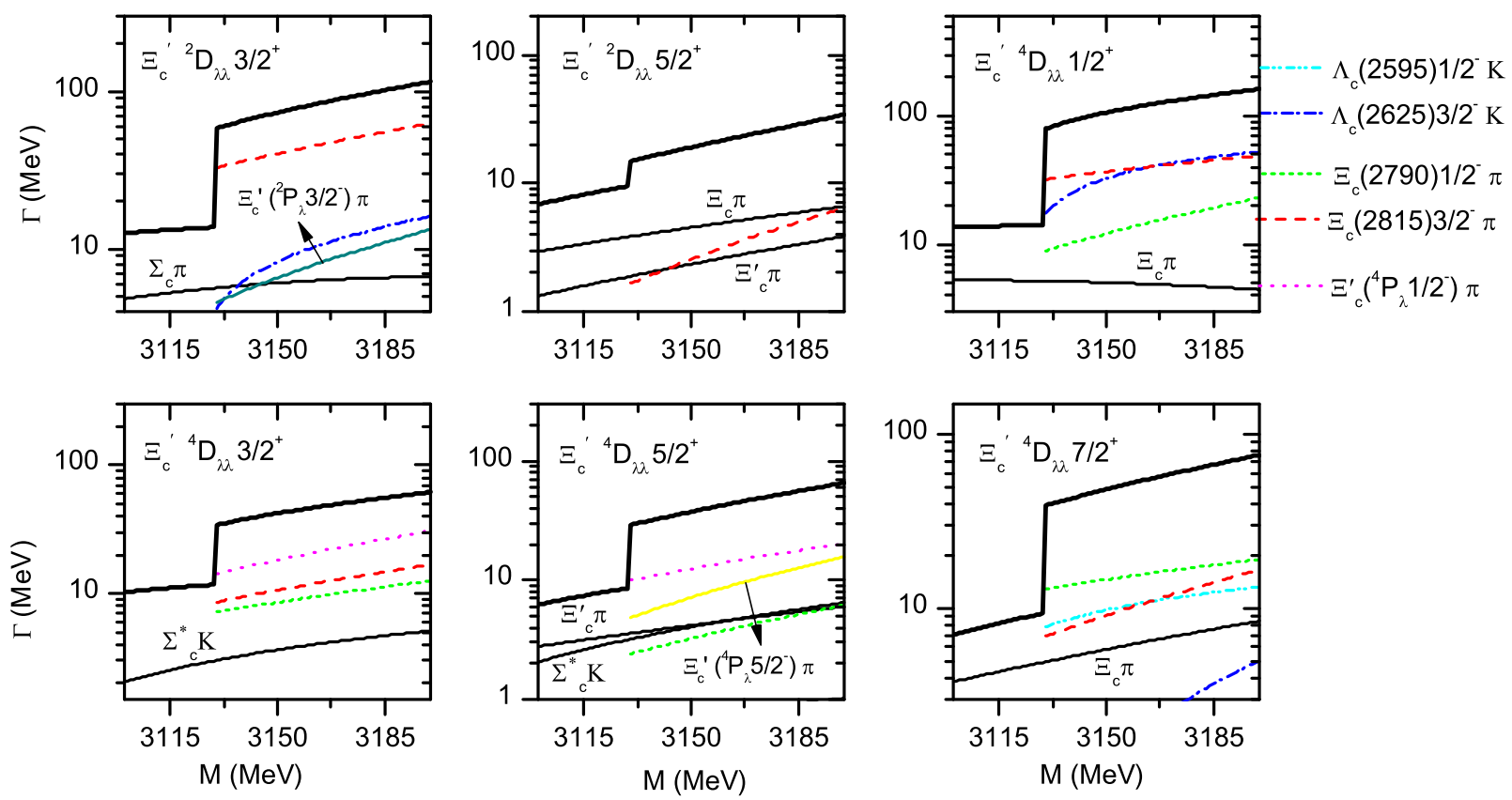

FIG. 5. Strong decay partial widths of the main decay modes for the $\lambda$-mode $1 D$-wave excited $\Xi_{c}^{\prime}$ states as functions of their mass. The bold solid curves stand for the sum of the partial widths. The masses of the $P$-wave heavy baryons in final states are adopted from the quark model predictions in Ref. [21], if there are no observations (see Table II).

specific, taking the masses of the $1 D$-wave states predicted within the relativistic quark-diquark picture [21], we give our predictions in Table X.

\section{1. $J^{P}=1 / 2^{+}$state}

If the mass of the $J^{P}=1 / 2^{+}$state $\left|\Xi_{c}^{\prime 4} D_{\lambda \lambda}{ }^{\frac{1}{2}}{ }^{+}\right\rangle$is less than $3.12 \mathrm{GeV}$, this state should be a rather narrow state with a width of $\sim 10 \mathrm{MeV}$; its main decay modes are $\Xi_{c} \pi, \Lambda_{c} K$, and $\Sigma_{c} K$. However, if the mass of $\left|\Xi_{c}^{\prime 4} D_{\lambda \lambda 2^{2}}{ }^{+}\right\rangle$is taken as the prediction $3163 \mathrm{MeV}$ in Ref. [21], the decay channels into the $P$-wave charmed baryon final states should open, and the decay channels $\Lambda_{c}(2625) K$ and $\Xi_{c}(2815) \pi$ will play dominant roles. In this case, the $J^{P}=1 / 2^{+}$state $\left|\Xi_{c}^{\prime 4} D_{\lambda \lambda^{2}}{ }^{+}\right\rangle$might be a broad state, and the sum of the partial widths for the pionic and kaonic decays can reach up to $\Gamma_{\text {Sum }} \sim 120 \mathrm{MeV}$ (see Table X), while the ratios between the partial widths for the $\Lambda_{c}(2625) K$ and $\Xi_{c}(2815) \pi$ channels and $\Gamma_{\text {Sum }}$ are predicted to be

$\frac{\Gamma\left[\Lambda_{c}(2625) K\right]}{\Gamma_{\text {Sum }}} \simeq 32 \%, \quad \frac{\Gamma\left[\Xi_{c}(2815) \pi\right]}{\Gamma_{\text {Sum }}} \simeq 33 \%$.

The decay channels $\Lambda_{c}(2625) K$ and $\Xi_{c}(2815) \pi$ may be ideal channels for our search for $\left|\Xi_{c}^{\prime 4} D_{\lambda \lambda}{ }^{+}{ }^{+}\right\rangle$in future experiments.

We also estimate its radiative decays into the $1 P$-wave bottom baryon states. Our results are listed in Table XI. It is found that $\left|\Xi_{c}^{\prime 04} D_{\lambda \lambda} \frac{1}{2}\right\rangle$ might have relatively large decay rates into $\left|\Xi_{c}^{\prime 04} P_{\lambda \frac{1}{2}}{ }^{-}\right\rangle \gamma$, and the partial radiative decay width is estimated to be $\mathcal{O}(100) \mathrm{keV}$. The branching fractions for this main radiative decay process may reach up to $\mathcal{O}\left(10^{-3}\right)$.

\section{2. $J^{P}=3 / 2^{+}$states}

For $\left|\Xi_{c}^{\prime 2} D_{\lambda \lambda \frac{3}{2}}{ }^{+}\right\rangle$, if its mass is less than $3.12 \mathrm{GeV}$, it should be a rather narrow state with a width of $\sim 10 \mathrm{MeV}$, and its main decay modes are $\Xi_{c} \pi, \Xi_{c}^{\prime} \pi$, and $\Sigma_{c} K$. However, if the mass of $\left|\Xi_{c}^{\prime 2} D_{\lambda \lambda^{2}}{ }^{+}\right\rangle$is taken as the prediction $3167 \mathrm{MeV}$ in Ref. [21], the decay channels into the $P$ wave charmed baryon final states should open, and the decay channels $\Lambda_{c}(2625) K$ and $\Xi_{c}(2815) \pi$ will play dominant roles. In this case, the $J^{P}=3 / 2^{+}$state $\left|\Xi_{c}^{\prime 2} D_{\lambda \lambda^{3}}{ }^{+}\right\rangle$might be a broad state, and the sum of the partial widths for the pionic and kaonic decays can reach up to $\Gamma_{\text {Sum }} \sim 90 \mathrm{MeV}$ (see Table X), while the ratios between the partial widths for the $\Lambda_{c}(2625) K$ and $\Xi_{c}(2815) \pi$ channels and $\Gamma_{\text {Sum }}$ are predicted to be

$\frac{\Gamma\left[\Lambda_{c}(2625) K\right]}{\Gamma_{\text {Sum }}} \simeq 12 \%, \quad \frac{\Gamma\left[\Xi_{c}(2815) \pi\right]}{\Gamma_{\text {Sum }}} \simeq 54 \%$.

The $\Lambda_{c}(2625) K$ and $\Xi_{c}(2815) \pi$ decay channels may be ideal channels for our search for $\left|\Xi_{c}^{\prime 2} D_{\lambda \lambda^{2}}{ }^{+}\right\rangle$in future experiments.

For the other $J^{P}=3 / 2^{+}$state $\left|\Xi_{c}^{\prime 4} D_{\lambda \lambda^{3}}{ }^{+}\right\rangle$, if its mass is less than $3.12 \mathrm{GeV}$, it should be a rather narrow state with a width of $\sim 10 \mathrm{MeV}$ as well, and its main decay modes are $\Xi_{c} \pi, \Xi_{c}^{\prime *} \pi$, and $\Sigma_{c}^{*} K$. However, if the mass of $\left|\Xi_{c}^{\prime}{ }^{4} D_{\lambda \lambda}{ }^{3}{ }^{+}\right\rangle$is taken as the prediction $3160 \mathrm{MeV}$ in Ref. [21], the decay 
TABLE X. Partial widths of strong decays for the $\lambda$-mode $D$-wave $\Xi_{c}^{\prime}$ and $\Xi_{b}^{\prime}$ baryons, the masses (MeV) of which are taken from the quark model predictions of Ref. [21]. $M_{f}$ stands for the masses of $P$-wave heavy baryons $(\mathrm{MeV})$ in the final states, which are adopted from the RPP [24] and Ref. [21]. The superscript (subscript) stands for the uncertainty of a prediction with a $+10 \%(-10 \%)$ uncertainty of the oscillator parameter $\alpha_{\rho}$.

\begin{tabular}{|c|c|c|c|c|c|c|c|}
\hline & & $\left|\Xi_{c}^{\prime 2} D_{\lambda \lambda^{\frac{3}{2}}}{ }^{+}\right\rangle(3167)$ & $\left|\Xi_{c}^{\prime 2} D_{\lambda \lambda 2^{2}}{ }^{5}\right\rangle(3166)$ & $\left|\Xi_{c}^{\prime 4} D_{\lambda \lambda \frac{1}{2}}{ }^{+}\right\rangle(3163)$ & $\left|\Xi_{c}^{\prime 4} D_{\lambda \lambda 2^{2}}{ }^{+}\right\rangle(3160)$ & $\left|\Xi_{c}^{\prime 4} D_{\lambda \lambda 2^{2}}{ }^{5}\right\rangle(3153)$ & $\left|\Xi_{c}^{\prime 4} D_{\lambda \lambda \lambda^{\frac{7}{2}}}\right\rangle(3147)$ \\
\hline Decay mode & $M_{f}$ & $\Gamma_{i}(\mathrm{MeV})$ & $\Gamma_{i}(\mathrm{MeV})$ & $\Gamma_{i}(\mathrm{MeV})$ & $\Gamma_{i}(\mathrm{MeV})$ & $\Gamma_{i}(\mathrm{MeV})$ & $\Gamma_{i}(\mathrm{MeV})$ \\
\hline$\Xi_{c} \pi$ & 2470 & $2.43_{-159}^{+2.13}$ & $5.13_{+1.05}^{-0.96}$ & $4.92_{-3.19}^{+4.18}$ & $2.48_{-1.60}^{+2.06}$ & $1.32_{+0.29}^{-0.25}$ & $5.68_{+1.24}^{-1.10}$ \\
\hline$\Xi_{c}^{\prime} \pi$ & 2578 & $3.51_{-1.66}^{+1.79}$ & $2.77_{+0.77}^{-0.02}$ & $1.75_{-0.81}^{+0.88}$ & $0.87_{-0.40}^{+0.44}$ & $0.17_{+0.05}^{-0.03}$ & $0.73_{+0.21}^{-0.17}$ \\
\hline$\Xi_{c}^{\prime *} \pi$ & 2645 & $0.74_{+0.13}^{-0.09}$ & $1.18_{-0.26}^{+0.27}$ & $0.76_{-0.28}^{+0.27}$ & $2.94_{-0.69}^{+0.70}$ & $4.28_{-0.24}^{+0.34}$ & $0.56_{-0.04}^{+0.06}$ \\
\hline$\Lambda_{c} K$ & 2286 & $1.23_{-0.83}^{+1.13}$ & $1.25_{+0.53}^{-0.43}$ & $2.51_{-1.67}^{+2.24}$ & $1.27_{-0.84}^{+1.12}$ & $0.72_{+0.15}^{-0.13}$ & $3.09_{+0.63}^{-0.58}$ \\
\hline$\Sigma_{c} K$ & 2455 & $6.37_{-2.07}^{-0.83}$ & $1.72_{+0.58}^{-0.43}$ & $3.16_{-1.0}^{+0.96}$ & $1.57_{-0.49}^{+0.46}$ & $0.11_{+0.04}^{-0.03}$ & $0.40_{+0.14}^{-0.10}$ \\
\hline$\Sigma_{c}^{*} K$ & 2520 & $0.47_{+0.04}^{-0.01}$ & $1.57_{-0.28}^{+0.25}$ & $1.22_{-0.26}^{+0.03}$ & $3.97_{-0.70}^{+0.62}$ & $4.18_{-0.40}^{+0.37}$ & $0.59_{-0.08}^{+0.08}$ \\
\hline$\left|\Lambda_{c}^{2} P_{\lambda 2^{-}}{ }^{-}\right\rangle K$ & 2592 & $0.84_{-0.10}^{+0.11}$ & $0.55_{-0.06}^{+0.08}$ & $3.66_{+0.38}^{-0.30}$ & $3.55_{-1.06}^{+1.22}$ & $0.65_{+0.07}^{-0.05}$ & $10.1_{-2.3}^{+2.5}$ \\
\hline$\left|\Lambda_{c}^{2} P_{\lambda^{\frac{3}{2}}-}^{-}\right\rangle K$ & 2628 & $10.9_{-2.1}^{+2.4}$ & $0.24_{-0.05}^{+0.10}$ & $39.1_{-8.6}^{+9.5}$ & $7.25_{-1.36}^{+1.53}$ & $0.15_{-0.02}^{+0.02}$ & $1.15_{-0.13}^{+0.15}$ \\
\hline$\left|\Xi_{c}^{2} P_{\lambda^{\frac{1}{2}}-}\right\rangle \pi$ & 2792 & $1.85_{-0.13}^{+0.15}$ & $1.83_{-0.13}^{+0.03}$ & $14.5_{+2.11}^{-1.28}$ & $9.28_{-1.43}^{+1.50}$ & $3.37_{+0.36}^{-0.02}$ & $14.4_{-3.5}^{-0.13}$ \\
\hline$\left|\Xi_{c}^{2} P_{\lambda^{2}}^{3-}\right\rangle \pi$ & 2815 & $47.3_{-7.3}^{+7.7}$ & $3.54_{+1.13}^{-0.13}$ & $40.2_{-10.5}^{+11.9}$ & $11.8_{-1.4}^{+1.7}$ & $0.67_{-0.02}^{+0.03}$ & $8.81_{-0.04}^{+0.04}$ \\
\hline$\left.\left|\Xi_{c}^{\prime 2} P_{\lambda 1^{1}}\right\rangle\right\rangle$ & 2936 & $0.39_{-0.05}^{+0.05}$ & $0.21_{+0.04}^{-0.03}$ & $0.26_{<+0.01}^{-0.03}$ & $0.51_{-0.14}^{+0.4}$ & 0.03 & $0.92_{-0.19}^{+0.04}$ \\
\hline$\left|\Xi_{c}^{\prime 2} P_{\lambda}{ }^{\frac{3}{2}}-\right\rangle \pi$ & 2935 & $8.53_{-1.50}^{+1.70}$ & $0.22_{+0.04}^{-0.03}$ & $5.50_{-1.21}^{+1.33}$ & $1.0_{-0.11}^{+0.13}$ & $<0.01$ & $0.33_{-0.02}^{+0.02}$ \\
\hline 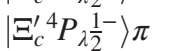 & 2854 & $0.23_{+0.10}^{-0.07}$ & $1.00_{-0.30}^{+0.32}$ & $0.75_{+0.14}^{-0.11}$ & $20.5_{-2.6}^{+2.9}$ & $13.0_{-2.0}^{+2.1}$ & $<0.01$ \\
\hline$\left|\Xi_{c}^{\prime 4} P_{\lambda}{ }^{\frac{3}{2}}-\right\rangle \pi$ & 2912 & $1.01_{-0.07}^{+0.10}$ & $0.41_{-0.04}^{+0.04}$ & $1.10_{-0.02}^{+0.03}$ & $5.62_{-0.72}^{+0.82}$ & $2.85_{-0.94}^{+1.08}$ & $0.34_{<+0.01}^{<-0.01}$ \\
\hline$\left|\Xi_{c}^{\prime 4} P_{\lambda{ }^{\frac{5}{2}}}\right\rangle \pi$ & 2929 & $0.59_{-0.02}^{+0.04}$ & $1.46_{-0.18}^{+0.22}$ & $2.23_{-0.57}^{+0.64}$ & $5.75_{-1.35}^{+1.51}$ & $7.55_{-1.05}^{+1.17}$ & $0.37_{+0.01}^{<-0.01}$ \\
\hline \multirow[t]{2}{*}{ Sum } & & $86.4_{-17.2}^{+19.1}$ & $23.1_{+2.8}^{-1.9}$ & $121.6_{-25.5}^{+30.4}$ & $78.4_{-14.9}^{+16.9}$ & $39.1_{-3.7}^{+1.3}$ & $47.5_{-4.1}^{+5.1}$ \\
\hline & & $\left|\Xi_{b}^{\prime 2} D_{\lambda \lambda}{ }^{\frac{3}{2}}\right\rangle(6459)$ & $\left|\Xi_{b}^{\prime 2} D_{\lambda \lambda \frac{5}{2}}{ }^{+}\right\rangle(6432)$ & $\left|\Xi_{b}^{\prime 4} D_{\lambda \lambda{ }^{2}}{ }^{1+}\right\rangle(6447)$ & $\left|\Xi_{b}^{\prime 4} D_{\lambda \lambda^{2}}{ }^{3}\right\rangle(6431)$ & $\left|\Xi_{b}^{\prime 4} D_{\lambda \lambda}{ }^{5}{ }^{+}\right\rangle(6420)$ & $\left|\Xi_{b}^{\prime 4} D_{\lambda \lambda}{ }^{7}{ }^{+}\right\rangle(6414)$ \\
\hline Decay mode & $M_{f}$ & $\Gamma_{i}(\mathrm{MeV})$ & $\Gamma_{i}(\mathrm{MeV})$ & $\Gamma_{i}(\mathrm{MeV})$ & $\Gamma_{i}(\mathrm{MeV})$ & $\Gamma_{i}(\mathrm{MeV})$ & $\Gamma_{i}(\mathrm{MeV})$ \\
\hline$\Xi_{b} \pi$ & 5795 & $2.02_{-1.72}^{+3.07}$ & $9.41_{+1.44}^{-1.51}$ & $4.41_{-3.64}^{+6.04}$ & $2.43_{-1.89}^{+2.93}$ & $2.42_{+0.40}^{-0.40}$ & $10.3_{+1.81}^{-1.73}$ \\
\hline$\Xi_{b}^{\prime} \pi$ & 5935 & $4.18_{-2.13}^{+2.37}$ & $2.82_{+0.81}^{-0.44}$ & $2.06_{-0.99}^{+3.104}$ & $1.01_{-0.46}^{+0.89}$ & $0.17_{+0.05}^{-0.04}$ & $0.71_{+0.22}^{+0.17}$ \\
\hline$\Xi_{b}^{\prime *} \pi$ & 5955 & $1.60_{+0.29}^{-0.13}$ & $1.59_{-0.35}^{+0.81}$ & $1.00_{-0.45}^{+0.49}$ & $4.03_{-0.99}^{+1.46}$ & $6.16_{-0.29}^{+0.03}$ & $0.79_{-0.03}^{+0.08}$ \\
\hline$\Lambda_{b} K$ & 5620 & $1.04_{-0.90}^{+1.67}$ & $2.45_{+0.83}^{-0.35}$ & $2.39_{-1.98}^{+3.45}$ & $1.40_{-1.19}^{+1.47}$ & $1.29_{+0.20}^{-0.29}$ & $5.45_{+0.90}^{-0.93}$ \\
\hline$\Sigma_{b} K$ & 5811 & $7.80_{-2.31}^{+2.16}$ & $0.86_{+0.33}^{-0.22}$ & $4.03_{-1.07}^{+0.98}$ & $1.83_{-0.42}^{+0.37}$ & 0.04 & $0.16_{+0.06}^{-0.04}$ \\
\hline$\Sigma_{b}^{*} K$ & 5835 & $0.85_{+0.08}^{-0.04}$ & $1.95_{-0.32}^{+0.28}$ & $1.77_{-0.39}^{+0.34}$ & $5.11_{-0.85}^{+0.74}$ & $4.77_{-0.45}^{+0.41}$ & $0.68_{-0.09}^{+0.08}$ \\
\hline$\left|\Lambda_{b}^{2} P_{\lambda} \lambda^{\frac{1}{2}}-\right\rangle K$ & 5912 & $0.77_{-0.10}^{+0.11}$ & 0.06 & $1.54_{+0.14}^{-0.10}$ & $3.18_{-0.74}^{-0.85}$ & 0.04 & $3.65_{-0.71}^{+0.79}$ \\
\hline$\left|\Lambda_{b}^{2} P_{\lambda{ }^{3}}{ }^{-}\right\rangle K$ & 5920 & $14.6_{-2.8}^{-0.07}$ & $0.17_{-0.07}^{+0.10}$ & $30.2_{-6.65}^{+7.48}$ & $5.87_{-1.13}^{+1.25}$ & 0.06 & $\ldots$ \\
\hline$\left|\Xi_{b}^{2} P_{\lambda}{ }^{1-}{ }^{-}\right\rangle \pi$ & 6120 & $1.90_{-0.11}^{+0.14}$ & $1.35_{-0.09}^{+0.12}$ & $13.2_{+1.28}^{-1.05}$ & $7.36_{-1.13}^{+1.22}$ & $2.15_{+0.25}^{-0.20}$ & $11.0_{-2.6}^{+3.0}$ \\
\hline$\left|\Xi_{b}^{2} P_{\lambda 2^{3}}-\right\rangle \pi$ & 6130 & $54.4_{-6.92}^{+8.22}$ & $2.80_{+0.93}^{-0.06}$ & $39.3_{-10.5}^{+12.0}$ & $10.5_{-1.29}^{+1.47}$ & $0.56_{<-0.01}^{+0.03}$ & $6.79_{<-0.01}^{+0.07}$ \\
\hline$\left|\Xi_{b}^{\prime 2} P_{\lambda^{2}}^{1-}\right\rangle \pi$ & 6233 & $0.47_{-0.05}^{-6.92}$ & $0.10_{+0.02}^{-0.01}$ & $\begin{array}{l}0.28_{+0.04}^{-10.03} \\
\end{array}$ & $0.43_{-0.11}^{+0.11}$ & 0.01 & $0.65_{-0.14}^{+0.14}$ \\
\hline$\left|\Xi_{b}^{\prime 2} P_{\lambda 2^{2}}\right\rangle$ & 6234 & $9.60_{-1.66}^{+1.87}$ & $0.14_{-0.02}^{+0.04}$ & $5.33_{-1.18}^{+1.31}$ & $0.72_{-0.09}^{+0.11}$ & 0.001 & $0.18_{-0.012}^{+0.01}$ \\
\hline$\left|\Xi_{b}^{\prime}{ }^{4} P_{\lambda^{2}}{ }^{1-}\right\rangle \pi$ & 6227 & 0.03 & $0.43_{-0.10}^{+0.02}$ & $0.13_{+0.02}^{-1.02}$ & $5.80_{-0.87}^{+0.09}$ & $3.35_{-0.56}^{+0.61}$ & $<0.01$ \\
\hline$\left|\Xi_{b}^{\prime 4} P_{\lambda{ }^{3}}{ }^{-}\right\rangle \pi$ & 6224 & $0.89_{-0.05}^{+0.08}$ & $0.21_{-0.03}^{+0.03}$ & $0.81_{-0.01}^{+0.03}$ & $3.16_{-0.43}^{+0.49}$ & $1.80_{-0.52}^{+0.56}$ & $0.12_{<-0.01}^{<+0.01}$ \\
\hline$\left|\Xi_{b}^{\prime 4} P_{\lambda}{ }^{\frac{5}{2}}-\right\rangle \pi$ & 6226 & $0.72_{<-0.01}^{+0.03}$ & $0.96_{-0.13}^{+0.14}$ & $2.21_{-0.58}^{+0.01}$ & $4.80_{-1.12}^{+1.43}$ & $5.26_{-0.76}^{+0.89}$ & $0.20_{<-0.01}^{+0.01}$ \\
\hline Sum & & $100.8_{-18.4}^{+22.6}$ & $25.3_{+3.3}^{-2.13}$ & $108.7_{-26.0}^{+32.4}$ & $57.6_{-12.7}^{+14.7}$ & $28.1_{-1.7}^{+2.10}$ & $40.7_{-4.6}^{+1.3}$ \\
\hline
\end{tabular}

channels into the $P$-wave charmed baryon final states should open, and the decay channels $\Lambda_{c}(2625) K$, $\Xi_{c}(2790) \pi, \Xi_{c}(2815) \pi$, and $\left|\Xi_{c}^{\prime 4} P_{\lambda}{ }^{\frac{1}{2}}\right\rangle \pi$ will dominate the decays of $\left|\Xi_{c}^{\prime 4} D_{\lambda \lambda^{3}}{ }^{+}\right\rangle$. In this case, the sum of the partial widths for the pionic and kaonic decays can reach up to $\Gamma_{\text {Sum }} \sim 80 \mathrm{MeV}$ (see Table X), while the ratios between the partial widths for the $\Lambda_{c}(2625) K, \Xi_{c}(2790) \pi$, and $\Xi_{c}(2815) \pi$ channels and $\Gamma_{\text {Sum }}$ are predicted to be
$\frac{\Gamma\left[\Lambda_{c}(2625) K, \Xi_{c}(2790) \pi, \Xi_{c}(2815) \pi\right]}{\Gamma_{\text {Sum }}} \simeq 10 \%, 12 \%, 16 \%$.

The $\Lambda_{c}(2625) K, \Xi_{c}(2790) \pi$, and $\Xi_{c}(2815) \pi$ decay channels may be ideal channels for a search for $\left|\Xi_{c}^{\prime 4} D_{\lambda \lambda \frac{3}{2}}{ }^{+}\right\rangle$in future experiments. 
TABLE XI. Partial widths of radiative decays for the $\lambda$-mode $D$-wave $\Xi_{c}^{\prime}$ and $\Xi_{b}^{\prime}$ baryons, the masses (MeV) of which are taken from the quark model predictions of Ref. [21]. $M_{f}$ stands for the masses of $P$-wave heavy baryons $(\mathrm{MeV})$ in the final states, which are adopted from the RPP [24] and Ref. [21]. The superscript (subscript) stands for the uncertainty of a prediction with a $+10 \%(-10 \%)$ uncertainty of the oscillator parameter $\alpha_{\rho}$.

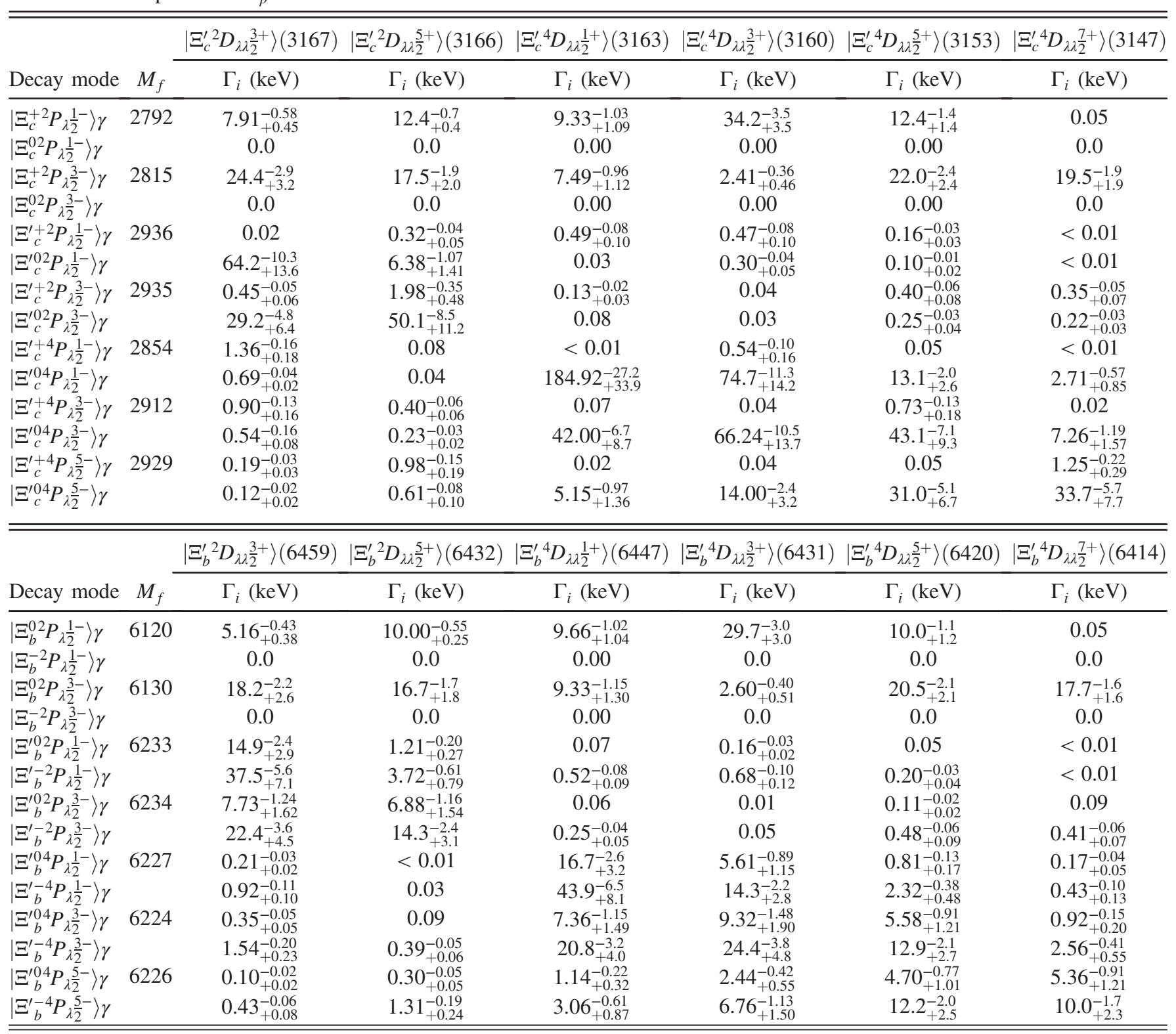

The radiative decays of these $J^{P}=3 / 2^{+}$states into the $1 P$-wave charmed baryon states are also estimated. Our results are listed in Table XI. It is found that for the $\left|\Xi_{c}^{\prime 2} D_{\lambda \lambda^{\frac{3}{2}}}{ }^{+}\right\rangle$state the main radiative decay processes are $\left|\Xi_{c}^{\prime+2} D_{\lambda \lambda^{3}}{ }^{+}\right\rangle \rightarrow \Xi_{c}^{+}(2815) \gamma, \quad\left|\Xi_{c}^{\prime 02} D_{\lambda \lambda^{3}}{ }^{+}\right\rangle \rightarrow\left|\Xi_{c}^{\prime 02} P_{\lambda{ }^{2}}{ }^{-}\right\rangle \gamma$, $\left|\Xi_{c}^{\prime 02} P_{\lambda{ }^{3}}{ }^{-}\right\rangle \gamma$, while for the $\left|\Xi_{c}^{\prime 4} D_{\lambda \lambda^{3}}{ }^{+}\right\rangle$state, the main radiative decay processes are $\left|\Xi_{c}^{\prime+4} D_{\lambda \lambda^{2}}{ }^{+}\right\rangle \rightarrow \Xi_{c}^{+}(2790) \gamma$, $\left|\Xi_{c}^{\prime 04} D_{\lambda \lambda}{ }^{\frac{3}{2}}\right\rangle \rightarrow\left|\Xi_{c}^{\prime 04} P_{\lambda{ }^{\frac{1}{2}}}{ }^{-}\right\rangle \gamma,\left|\Xi^{\prime 04} P_{\lambda}{ }^{\frac{3}{2}}\right\rangle \gamma$. Their partial radiative decay widths are estimated to be $\mathcal{O}(10) \mathrm{keV}$, while the branching fractions may reach up to $\mathcal{O}\left(10^{-4}\right)-$ $\mathcal{O}\left(10^{-3}\right)$.

\section{3. $J^{P}=5 / 2^{+}$states}

For $\left|\Xi_{c}^{\prime 2} D_{\lambda \lambda \overline{2}}{ }^{+}\right\rangle$, if its mass is less than $3.12 \mathrm{GeV}$, it should be a rather narrow state with a width of $\sim 10 \mathrm{MeV}$, and its main decay modes are $\Xi_{c} \pi, \Xi_{c}^{\prime} \pi$. However, if the mass of $\left|\Xi_{c}^{\prime 2} D_{\lambda \lambda^{3}}{ }^{+}\right\rangle$is taken as the prediction $3166 \mathrm{MeV}$ in Ref. [21], the decay channels into the $P$-wave charmed baryon final states should open, and the $\Xi_{c}(2815) \pi$ decay mode together with $\Xi_{c} \pi$ and $\Xi_{c}^{\prime} \pi$ dominates its decays. In this case, the width of $\left|\Xi_{c}^{\prime 2} D_{\lambda \lambda}{ }^{\frac{5}{2}}\right\rangle$ might be still fairly narrow, and the sum of the partial widths for the pionic and kaonic decays is $\Gamma_{\text {Sum }} \sim 20 \mathrm{MeV}$ (see Table X), while the 
ratios between the partial widths for the $\Xi_{c} \pi, \Xi_{c}^{\prime} \pi$, and $\Xi_{c}(2815) \pi$ channels and $\Gamma_{\text {Sum }}$ are predicted to be

$$
\frac{\Gamma\left[\Xi_{c} \pi, \Xi_{c}^{\prime} \pi, \Xi_{c}(2815) \pi\right]}{\Gamma_{\text {Sum }}} \simeq 22 \%, 12 \%, 15 \% .
$$

The $\Xi_{c} \pi, \Xi_{c}^{\prime} \pi$, and $\Xi_{c}(2815) \pi$ decay modes may be ideal modes for our search for $\left|\Xi_{c}^{\prime 2} D_{\lambda \lambda^{2}}{ }^{+}\right\rangle$in future experiments.

For the other $J^{P}=5 / 2^{+}$state $\left|\Xi_{c}^{\prime}{ }^{4} D_{\lambda \lambda^{2}}{ }^{+}\right\rangle$, if its mass is less than $3.12 \mathrm{GeV}$, it should be a rather narrow states with a width of $\sim 10 \mathrm{MeV}$ as well, its main decay modes are $\Xi_{c}^{\prime *} \pi$ and $\Sigma_{c}^{*} K$. However, if the mass of $\left|\Xi_{c}^{\prime 4} D_{\lambda \lambda} \lambda^{\frac{5}{2}}\right\rangle$ is taken as the prediction $3153 \mathrm{MeV}$ in Ref. [21], the decay channels into the $P$-wave charmed baryon final states should open, and the $\Xi_{c}(2790) \pi,\left|\Xi_{c}^{\prime 4} P_{\lambda^{2}}{ }^{1-}\right\rangle \pi$, and $\left|\Xi_{c}^{\prime{ }^{4}} P_{\lambda^{\frac{5}{2}}}{ }^{-}\right\rangle \pi$ decay modes together with $\Xi_{c}^{\prime *} \pi$ and $\Sigma_{c}^{*} K$ will dominate the decays of $\left|\Xi_{c}^{\prime 4} D_{\lambda \lambda^{\frac{5}{2}}}{ }^{+}\right\rangle$. In this case, the sum of the partial widths for the pionic and kaonic decays can reach up to $\Gamma_{\text {Sum }} \sim$ $40 \mathrm{MeV}$ (see Table X), while the ratios between the partial widths for the $\Xi_{c}^{\prime *} \pi, \Sigma_{c}^{*} K$, and $\Xi_{c}(2790) \pi$ channels and $\Gamma_{\text {Sum }}$ are predicted to be

$$
\frac{\Gamma\left[\Xi_{c}^{*} \pi, \Sigma_{c}^{*} K, \Xi_{c}(2790) \pi\right]}{\Gamma_{\text {Sum }}} \simeq 11 \%, 11 \%, 9 \%
$$

The decay modes $\Xi_{c}^{* *} \pi, \Sigma_{c}^{*} K$, and $\Xi_{c}(2790) \pi$ may be ideal modes for our search for $\left|\Xi_{c}^{\prime 4} D_{\lambda \lambda^{2}}{ }^{+}\right\rangle$in future experiments.

The radiative decays of these $J^{P}=5 / 2^{+}$states into the $1 P$-wave charmed baryon states are also estimated. Our results are listed in Table XI. It is found that for the $\left|\Xi_{c}^{\prime 2} D_{\lambda \lambda}{ }^{\frac{5}{2}}\right\rangle$ state the main radiative decay processes are $\quad\left|\Xi_{c}^{\prime+2} D_{\lambda \lambda} \lambda^{\frac{5}{2}}\right\rangle \rightarrow \Xi_{c}^{+}(2790,2815) \gamma, \quad\left|\Xi_{c}^{\prime 02} D_{\lambda \lambda} \frac{5}{2}\right\rangle \rightarrow$ $\left|\Xi_{c}^{\prime 02} P_{\lambda^{2}}{ }^{3}\right\rangle \gamma$, while for the $\left|\Xi_{c}^{\prime 4} D_{\lambda \lambda^{2}}{ }^{+}\right\rangle$state, the main radiative decay processes are $\left|\Xi_{c}^{\prime+4} D_{\lambda \lambda^{2}}{ }^{+}\right\rangle \rightarrow \Xi_{c}^{+}(2790$, 2815) $\gamma, \quad\left|\Xi_{c}^{\prime 04} D_{\lambda \lambda \lambda^{\frac{3}{2}}}\right\rangle \rightarrow\left|\Xi^{\prime 04} P_{\lambda^{2}}{ }^{\frac{1}{}}\right\rangle \gamma, \quad\left|\Xi_{c}^{\prime 04} P_{\lambda^{2}}{ }^{-}\right\rangle \gamma$, $\left|\Xi^{\prime 04} P_{\lambda}{ }^{5}-\right\rangle \gamma$. Their partial radiative decay widths are estimated to be $\mathcal{O}(10) \mathrm{keV}$, while the branching fractions may reach up to $\mathcal{O}\left(10^{-4}\right)-\mathcal{O}\left(10^{-3}\right)$.

\section{4. $J^{P}=7 / 2^{+}$state}

If the mass of the $J^{P}=7 / 2^{+}$state $\left|\Xi_{c}^{\prime 4} D_{\lambda \lambda \frac{7}{2}}{ }^{+}\right\rangle$is less than $3.12 \mathrm{GeV}$, it should be a rather narrow state with a width of a few $\mathrm{MeV}$, and its main decay modes are $\Xi_{c} \pi$ and $\Lambda_{c} K$. However, if the mass of $\left|\Xi_{c}^{\prime 4} D_{\lambda \lambda^{2}}{ }^{+}\right\rangle$is taken to be $3147 \mathrm{MeV}$ as predicted in Ref. [21], the decay channels into the $P$-wave charmed baryon final states, $\Lambda_{c}(2595) K$, $\Xi_{c}(2790) \pi$, and $\Xi_{c}(2815) \pi$, become dominant. In this case, the sum of the partial widths for the pionic and kaonic decays can reach up to $\Gamma_{\text {Sum }} \sim 50 \mathrm{MeV}$ (see Table X), while the ratios between the partial widths for the main channels and $\Gamma_{\text {Sum }}$ are predicted to be

$$
\begin{aligned}
& \frac{\Gamma\left[\Xi_{c} \pi, \Lambda_{c}(2595) K, \Xi_{c}(2790) \pi, \Xi_{c}(2815) \pi\right]}{\Gamma_{\text {Sum }}} \\
& \quad \simeq 12 \%, 20 \%, 30 \%, 19 \% .
\end{aligned}
$$

The $\Xi_{c} \pi, \Lambda_{c} K, \Lambda_{c}(2595) K, \Xi_{c}(2790) \pi$, and $\Xi_{c}(2815) \pi$ decay modes may be ideal modes for our search for $\left|\Xi_{c}^{\prime 4} D_{\lambda \lambda \frac{7}{2}}{ }^{7}\right\rangle$ in future experiments.

The radiative decays of $\left|\Xi_{c}^{\prime 4} D_{\lambda \lambda^{2}}{ }^{+}\right\rangle$into the $1 P$-wave charmed baryon states are also estimated. Our results are listed in Table XI. It is found that the main radiative decay processes are $\left|\Xi_{c}^{\prime+2} D_{\lambda \lambda^{2}}{ }^{+}\right\rangle \rightarrow \Xi_{c}^{+}(2815) \gamma,\left|\Xi_{c}^{\prime 02} D_{\lambda \lambda \overline{2}^{2}}\right\rangle \rightarrow$ $\left|\Xi^{\prime 02} P_{\lambda \overline{2}}{ }^{5}\right\rangle \gamma$. Their partial radiative decay widths are estimated to be $\mathcal{O}(10) \mathrm{keV}$, while the branching fractions may be $\mathcal{O}\left(10^{-4}\right)$.

\section{D. $\Xi_{b}^{\prime}$}

In the $\Xi_{b}^{\prime}$ family, there are six $\lambda$-mode $1 D$-wave excitations: $\quad\left|\Xi_{b}^{\prime 4} D_{\lambda \lambda \frac{1}{2}}{ }^{+}\right\rangle, \quad\left|\Xi_{b}^{\prime{ }^{4}} D_{\left.\lambda \lambda{ }^{\frac{3}{2}}{ }^{+}\right\rangle,}\right\rangle, \quad \mid \Xi_{b}^{\prime 2} D_{\left.\lambda \lambda^{\frac{3}{2}}{ }^{+}\right\rangle,}$ $\left|\Xi_{b}^{\prime 2} D_{\lambda \lambda^{2}}{ }^{+}\right\rangle,\left|\Xi_{b}^{\prime 4} D_{\lambda \lambda^{\frac{5}{2}}}\right\rangle$, and $\left|\Xi_{b}^{\prime 4} D_{\lambda \lambda^{2}}{ }^{+}\right\rangle$. However, no $1 D$-wave states have been established. The typical masses of the $\lambda$-mode $1 D$-wave $\Xi_{b}^{\prime}$ excitations are $\sim 6.44 \mathrm{GeV}$ within various quark model predictions (see Table II). In the possible mass ranges, we study their strong decay transitions by emitting one light pseudoscalar meson within the ChQM. Our results are shown in Fig. 6. To be more specific, taking the masses of the $1 D$-wave states obtained in the relativistic quark-diquark picture [21], we give the predicted widths in Table X.

\section{1. $J^{P}=1 / 2^{+}$state}

The $J^{P}=1 / 2^{+}$state $\left|\Xi_{b}^{\prime 4} D_{\lambda \lambda^{2}}{ }^{+}\right\rangle$might be a broad state with a width of $\mathcal{O}(100) \mathrm{MeV}$, which obviously depends on its mass. This state might mainly decay into the $P$-wave bottom baryons via pionic decay modes $\left|\Xi_{b}{ }^{2} P_{\lambda}{ }^{1-}\right\rangle \pi$ and $\left|\Xi_{b}{ }^{2} P_{\lambda \frac{3}{2}}{ }^{-}\right\rangle \pi$ and kaonic decay mode $\Lambda_{b}(5920) K$. If its mass is taken as the prediction $6447 \mathrm{MeV}$ in Ref. [21], the sum of the partial widths for the pionic and kaonic decays can reach up to $\Gamma_{\text {Sum }} \sim 110 \mathrm{MeV}$ (see Table VIII), while the ratio between the partial width for the $\Lambda_{b}(5920) K$ channel and $\Gamma_{\text {Sum }}$ is predicted to be

$$
\frac{\Gamma\left[\Lambda_{b}(5920) K\right]}{\Gamma_{\text {Sum }}} \simeq 27 \%
$$

The $\Lambda_{b}(5920) K$ decay channel may be an ideal channel for our search for $\left|\Xi_{b}^{\prime 4} D_{\lambda \lambda} \frac{1}{2}^{+}\right\rangle$in future experiments.

We also estimate its radiative decays into the $1 P$-wave bottom baryon states. Our results are listed in Table XI. It is found that $\left|\Xi_{b}^{\prime-4} D_{\lambda \lambda \frac{1}{2}}{ }^{+}\right\rangle$might have relatively large decay rates into $\left|\Xi_{b}^{\prime-4} P_{\lambda \frac{1}{2}}{ }^{-}\right\rangle \gamma$ and $\left|\Xi_{b}^{\prime-4} P_{\lambda^{2}}{ }^{-}\right\rangle \gamma$, and the partial radiative decay width is estimated to be $\mathcal{O}(10) \mathrm{keV}$. 

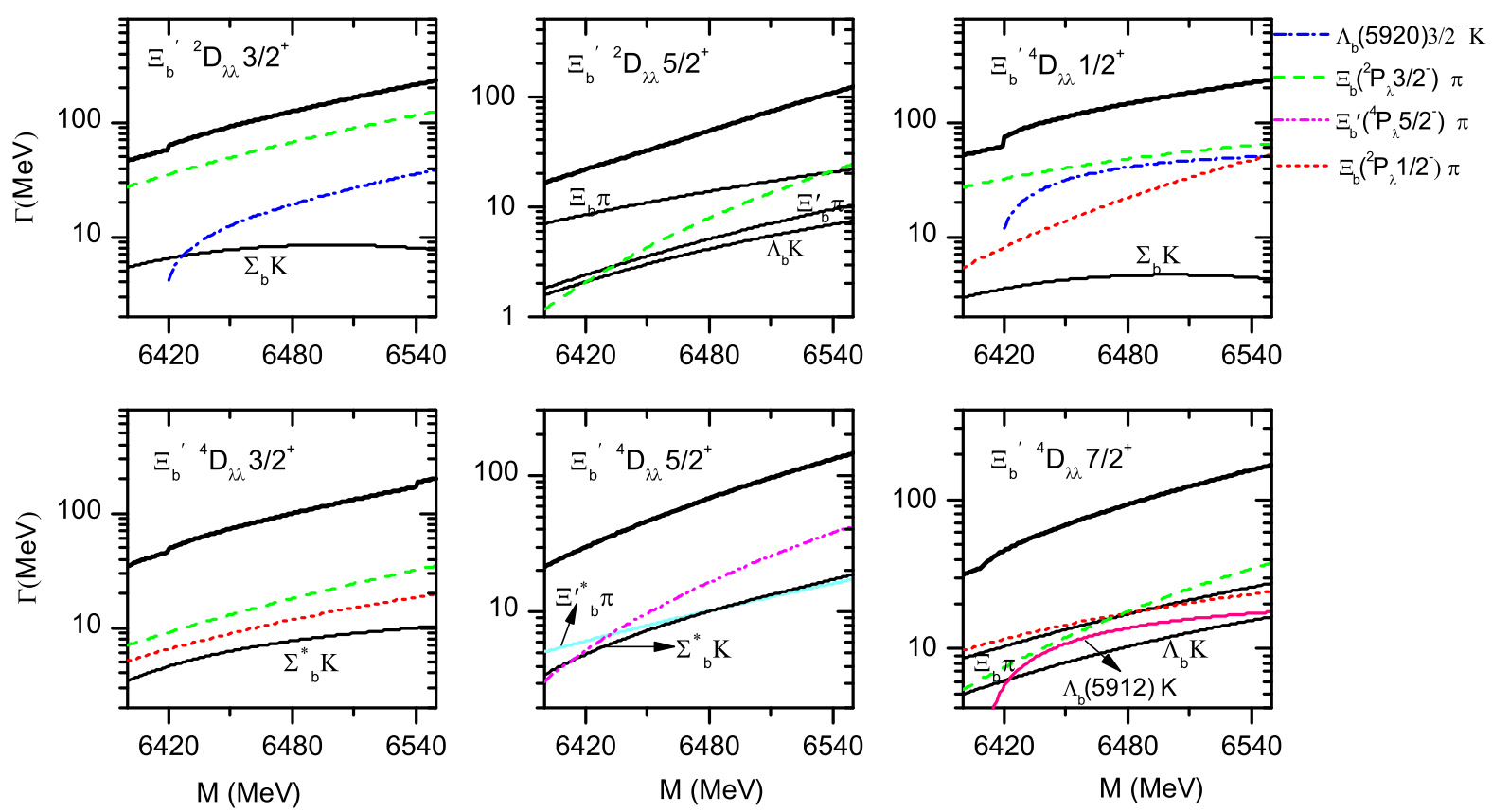

FIG. 6. Strong decay partial widths of the main decay modes for the $\lambda$-mode $1 D$-wave excited $\Xi_{b}^{\prime}$ states as functions of their mass. The bold solid curves stand for the sum of the partial widths. The masses of the $P$-wave heavy baryons in final states are adopted from the quark model predictions in Ref. [21], if there are no observations (see Table II).

The branching fractions for these main radiative decay processes may be $\mathcal{O}\left(10^{-4}\right)$.

\section{2. $J^{P}=3 / 2^{+}$states}

The $J^{P}=3 / 2^{+}$state $\left|\Xi_{b}^{\prime 2} D_{\lambda \lambda^{\frac{3}{2}}}{ }^{+}\right\rangle$has a width of $O(10)-O(100) \mathrm{MeV}$, which significantly depends on the mass. It might mainly decay into the $P$-wave bottom baryons via the $\left|\Xi_{b}{ }^{2} P_{\lambda^{2}}{ }^{-}\right\rangle \pi$ and $\Lambda_{b}(5920) K$ decay modes. The decay rate into $\Sigma_{b} K$ is also sizeable. If its mass is taken as the prediction in Ref. [21], the sum of the partial widths for the pionic and kaonic decays can reach up to $\Gamma_{\text {Sum }} \sim$ $100 \mathrm{MeV}$ (see Table X), while the ratios between the partial widths for the $\Sigma_{b} K$ and $\Lambda_{b}(5920) K$ channels and $\Gamma_{\text {Sum }}$ are predicted to be

$$
\frac{\Gamma\left[\Sigma_{b} K\right]}{\Gamma_{\text {Sum }}} \simeq 7 \%, \quad \frac{\Gamma\left[\Lambda_{b}(5920) K\right]}{\Gamma_{\text {Sum }}} \simeq 14 \% .
$$

The $\Sigma_{b} K$ and $\Lambda_{b}(5920) K$ decay modes may be ideal modes for our search for $\left|\Xi_{b}^{\prime 2} D_{\lambda \lambda^{\frac{3}{2}}}{ }^{+}\right\rangle$in future experiments.

For the other $J^{P}=3 / 2^{+}$state $\left|\Xi_{b}^{\prime 4} D_{\lambda \lambda \frac{3}{2}}{ }^{+}\right\rangle$, one finds that it has large decay rates into the $P$-wave states through the decay modes $\Lambda_{b}(5912) K, \quad \Lambda_{b}(5920) K, \quad\left|\Xi_{b}{ }^{2} P_{\lambda} \frac{1}{2}-\right\rangle$, $\left|\Xi_{b}{ }^{2} P_{\lambda^{2}}{ }^{-}\right\rangle \pi,\left|\Xi_{b}^{\prime 4} P_{\lambda^{\frac{1}{2}}}{ }^{-}\right\rangle \pi$, and $\left|\Xi_{b}^{\prime 4} P_{\lambda^{\frac{5}{2}}}{ }^{-}\right\rangle \pi$. Furthermore, the decay rates into $\Xi_{b}^{\prime *} \pi$ and $\Sigma_{b}^{*} K$ are sizable as well. Its width should be about a factor of 2 narrower than that of $\left|\Xi_{b}^{\prime 2} D_{\lambda \lambda \frac{3}{2}}{ }^{+}\right\rangle$. If its mass is taken as the predictions in Ref. [21], the sum of the partial widths of the pionic and kaonic decays can reach up to $\Gamma_{\text {Sum }} \sim 60 \mathrm{MeV}$ (see
Table VIII), while the ratios between the partial widths for the $\Xi_{b}^{\prime *} \pi, \Sigma_{b}^{*} K, \Lambda_{b}(5912) K$, and $\Lambda_{b}(5920) K$ decay modes and $\Gamma_{\text {Sum }}$ are predicted to be

$$
\begin{aligned}
& \frac{\Gamma\left[\Xi_{b}^{\prime *} \pi, \Sigma_{b}^{*} K, \Lambda_{b}(5912) K, \Lambda_{b}(5920) K\right]}{\Gamma_{\text {Sum }}} \\
& \quad \simeq 7 \%, 8 \%, 5 \%, 10 \% .
\end{aligned}
$$

The $\Xi_{b}^{\prime *} \pi, \Sigma_{b}^{*} K, \Lambda_{b}(5912) K$, and $\Lambda_{b}(5920) K$ decay modes may be ideal modes for our search for $\left|\Xi_{b}^{\prime} D_{\lambda \lambda^{\frac{3}{2}}}{ }^{+}\right\rangle$in future experiments.

We also estimate the radiative decays of these $J^{P}=$ $3 / 2^{+}$states into the $1 P$-wave bottom baryon states. Our results are listed in Table XI. It is found that for $\left|\Xi_{b}^{\prime 2} D_{\lambda \lambda^{2}}{ }^{+}\right\rangle$ the main radiative processes are $\left|\Xi_{b}^{\prime 02} D_{\lambda \lambda^{2}}{ }^{+}\right\rangle \rightarrow\left|\Xi_{b}^{02} P_{\lambda 2^{3}}{ }^{-}\right\rangle \gamma$ and $\left|\Xi_{b}^{\prime-2} D_{\lambda \lambda^{2}}{ }^{+}\right\rangle \rightarrow\left|\Xi_{b}^{\prime-2} P_{\lambda \frac{3}{2}}{ }^{-}\right\rangle \gamma,\left|\Xi_{b}^{\prime-2} P_{\lambda^{\frac{3}{2}}}{ }^{-}\right\rangle$, while for $\left|\Xi_{b}^{\prime 4} D_{\lambda \lambda 2^{3}}{ }^{+}\right\rangle$, the main radiative processes are $\left|\Xi_{b}^{\prime 04} D_{\lambda \lambda^{2}}{ }^{+}\right\rangle \rightarrow$ $\left|\Xi_{b}^{02} P_{\lambda^{2}}{ }^{-}\right\rangle \gamma$ and $\left|\Xi_{b}^{\prime-4} D_{\lambda \lambda^{2}}{ }^{+}\right\rangle \rightarrow\left|\Xi_{b}^{\prime-4} P_{\lambda \frac{1}{2}}{ }^{-}\right\rangle \gamma,\left|\Xi_{b}^{\prime-4} P_{\lambda \lambda^{\frac{3}{2}}}\right\rangle \gamma$. Their partial radiative decay widths are estimated to be $\mathcal{O}(10) \mathrm{keV}$, while the branching fractions may be $\mathcal{O}\left(10^{-4}\right)$.

\section{3. $J^{P}=5 / 2^{+}$states}

The $J^{P}=5 / 2^{+}$state $\left|\Xi_{b}^{\prime 2} D_{\lambda \lambda \frac{5}{2}}{ }^{+}\right\rangle$might be a narrow state with a width of a few tens of $\mathrm{MeV}$. Its dominant decay mode is $\Xi_{b} \pi$. If the mass of $\left|\Xi_{b}^{\prime 2} D_{\lambda \lambda^{2}}{ }^{+}\right\rangle$is taken as the prediction $6432 \mathrm{MeV}$ in Ref. [21], the sum of the partial widths for the pionic and kaonic decays is $\Gamma_{\text {Sum }} \sim 25 \mathrm{MeV}$ 
(see Table $\mathrm{X}$ ), while the ratio between the partial width for the $\Xi_{b} \pi$ channel and $\Gamma_{\text {Sum }}$ is predicted to be

$$
\frac{\Gamma\left[\Xi_{b} \pi\right]}{\Gamma_{\text {Sum }}} \simeq 37 \% \text {. }
$$

To look for this state, the $\Xi_{b} \pi$ decay mode is worth observing in future experiments.

The other $J^{P}=5 / 2^{+}$state $\left|\Xi_{b}^{\prime 4} D_{\lambda \lambda}{ }^{\frac{5}{2}}{ }^{+}\right\rangle$might be also a narrow state with a width of a few tens of $\mathrm{MeV}$. It has large decay rates into $\Xi_{b}^{\prime *} \pi$ and $\Sigma_{b}^{*} K$. If its mass is taken as the predictions in Ref. [21], the sum of the partial widths of the pionic and kaonic decays is $\Gamma_{\text {Sum }} \sim 30 \mathrm{MeV}$ (see Table VIII), while the ratios between the partial widths for the $\Xi_{b}^{* *} \pi$ and $\Sigma_{b}^{*} K$ decay modes and $\Gamma_{\text {Sum }}$ are predicted to be

$$
\frac{\Gamma\left[\Xi_{b}^{\prime *} \pi\right]}{\Gamma_{\text {Sum }}} \simeq 20 \%, \quad \frac{\Gamma\left[\Sigma_{b}^{*} K\right]}{\Gamma_{\text {Sum }}} \simeq 16 \% .
$$

To look for this state, the $\Xi_{b}^{\prime *} \pi$ and $\Sigma_{b}^{*} K$ decay modes are worth observing in future experiments.

We also estimate the radiative decays of these $J^{P}=$ $5 / 2^{+}$states into the $1 P$-wave bottom baryon states. Our results are listed in Table XI. It is found that for $\left|\Xi_{b}^{\prime 2} D_{\lambda \lambda^{2}}{ }^{+}\right\rangle$ the main radiative processes are $\left|\Xi_{b}^{\prime 02} D_{\lambda \lambda^{2}}{ }^{+}\right\rangle \rightarrow\left|\Xi_{b}^{02} P_{\lambda{ }^{3}}{ }^{-}\right\rangle \gamma$ and $\left|\Xi_{b}^{\prime-2} D_{\lambda \lambda \frac{5}{2}}{ }^{+}\right\rangle \rightarrow\left|\Xi_{b}^{\prime-2} P_{\lambda{ }^{3}}{ }^{-}\right\rangle \gamma$; while for $\left|\Xi_{b}^{\prime 4} D_{\lambda \lambda^{\frac{5}{2}}}{ }^{+}\right\rangle$, the main radiative processes are $\left|\Xi_{b}^{\prime 04} D_{\lambda \lambda}{ }^{\frac{5}{2}}{ }^{+}\right\rangle \rightarrow\left|\Xi_{b}^{02} P_{\lambda{ }^{2}}{ }^{-}\right\rangle \gamma$ and

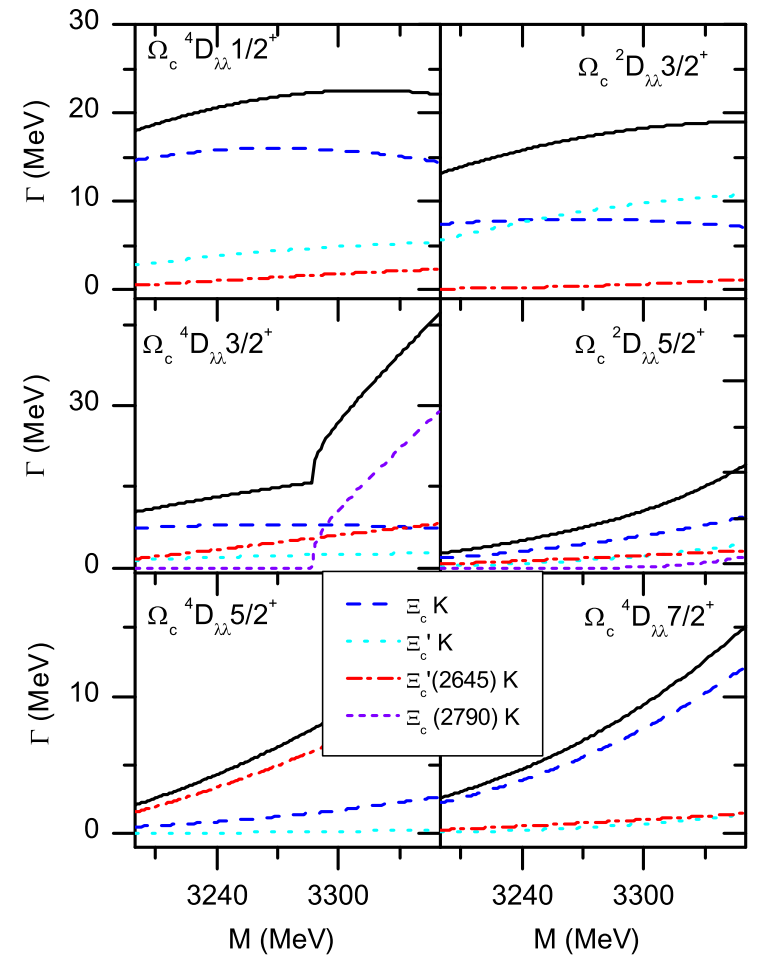

$\left|\Xi_{b}^{\prime-4} D_{\lambda \lambda}{ }^{\frac{5}{2}}\right\rangle \rightarrow\left|\Xi_{b}^{\prime-4} P_{\lambda{ }^{\frac{3}{2}}}{ }^{-}\right\rangle \gamma,\left|\Xi_{b}^{\prime-4} P_{\lambda{ }^{\frac{5}{2}}}\right\rangle \gamma$. Their partial radiative decay widths are estimated to be $\sim 10-20 \mathrm{keV}$, while the branching fractions is no more than $\mathcal{O}\left(10^{-4}\right)$.

\section{4. $J^{P}=7 / 2^{+}$state}

The $J^{P}=7 / 2^{+}$state $\left|\Xi_{b}^{\prime 4} D_{\lambda \lambda^{2}}{ }^{+}\right\rangle$might have a relatively narrow width of $\mathcal{O}(10) \mathrm{MeV}$. This state mainly decays into $\Xi_{b} \pi, \quad \Lambda_{b} K, \quad \Lambda_{b}(5912) K, \quad\left|\Xi_{b}{ }^{2} P_{\lambda^{2}}{ }^{-}\right\rangle \pi$, and $\left|\Xi_{b}{ }^{2} P_{\lambda^{2}}{ }^{-}\right\rangle \pi$ channels. If one adopts the predicted mass in Ref. [21], the sum of the partial widths of the pionic and kaonic decays is estimated to be $\Gamma_{\text {Sum }} \sim 40 \mathrm{MeV}$ (see Table VIII), and the ratios between the partial widths for the $\Xi_{b} \pi, \Lambda_{b} K, \Lambda_{b}(5912) K$ decay modes and $\Gamma_{\text {Sum }}$ are predicted to be

$$
\frac{\Gamma\left[\Xi_{b} \pi, \Lambda_{b} K, \Lambda_{b}(5912) K\right]}{\Gamma_{\text {Sum }}} \simeq 24 \%, 13 \%, 8 \% .
$$

The $\Xi_{b} \pi, \Lambda_{b} K$, and $\Lambda_{b}(5912) K$ decay modes may be ideal modes for our search for $\left|\Xi_{b}^{\prime 4} D_{\lambda \lambda^{2}}{ }^{+}\right\rangle$in future experiments.

We also estimate its radiative decays into the $1 P$-wave bottom baryon states. Our results are listed in Table XI. It is found that the decay rates for these radiative transitions are small. Their branching fractions may be no more than $\mathcal{O}\left(10^{-4}\right)$.

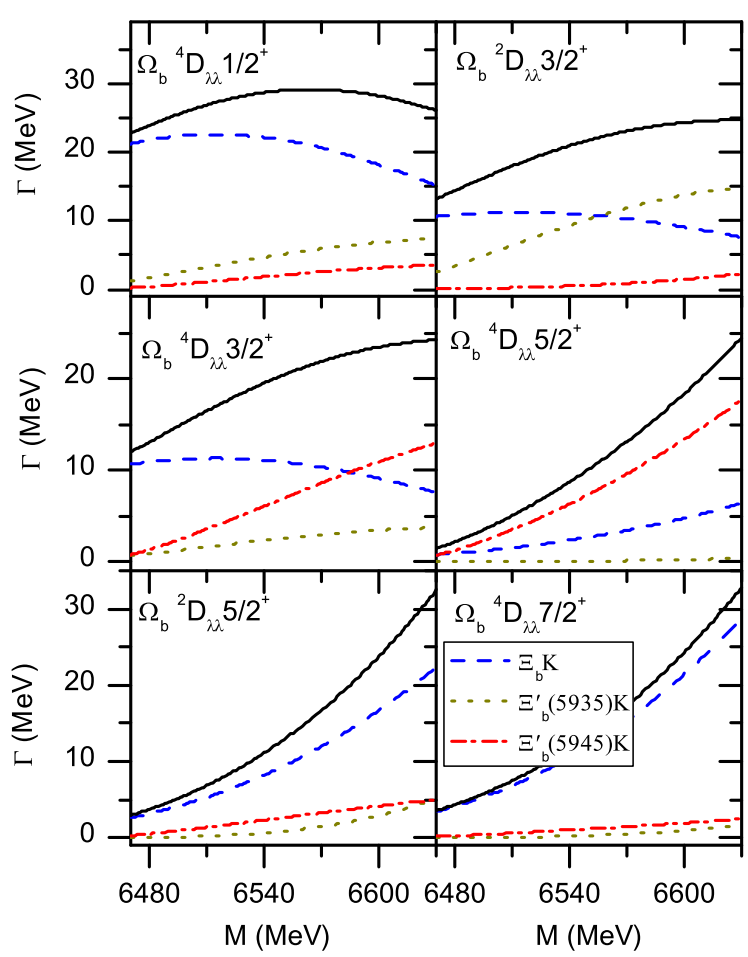

FIG. 7. Strong decay partial widths of the main decay modes for the $\lambda$-mode $1 D$-wave excited $\Omega_{c}$ and $\Omega_{b}$ states as functions of their mass. The bold solid curves stand for the total widths. 


\section{E. $\boldsymbol{\Omega}_{\boldsymbol{c}}$}

In the $\Omega_{c}$ family, there are six $\lambda$-mode $1 D$-wave excitations: $\quad\left|\Omega_{c}{ }^{4} D_{\lambda \lambda^{2}}{ }^{+}\right\rangle, \quad\left|\Omega_{c}{ }^{4} D_{\lambda \lambda \lambda^{\frac{3}{2}}}{ }^{+}\right\rangle, \quad\left|\Omega_{c}{ }^{2} D_{\lambda \lambda^{\frac{3}{2}}}{ }^{+}\right\rangle$,

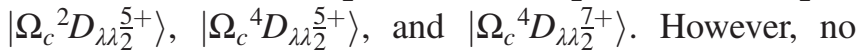
$D$-wave states have been established. The typical masses of the $\lambda$-mode $1 D$-wave $\Omega_{c}$ excitations are $\sim 3.3$ within various quark model predictions (see Table II). In the possible mass ranges, we study their strong decay transitions within the ChQM. Our results are shown in Fig. 7. To be more specific, taking the masses of the $1 D$-wave states obtained in the relativistic quark-diquark picture [21], we give the predicted widths in Table XII.

\section{1. $J^{P}=1 / 2^{+}$state}

The $J^{P}=1 / 2^{+}$state $\left|\Omega_{c}{ }^{4} D_{\lambda \lambda^{2}}{ }^{+}\right\rangle$may be a narrow state with a width of $\Gamma_{\text {total }} \sim 20 \mathrm{MeV}$. Its decays are dominated by the $\Xi_{c}(2470) K$. The decay rates into $\Xi_{c}^{\prime}(2575) K$ and $\Xi_{c}^{\prime *}(2645) K$ are sizeable as well. The branching fractions for the $\Xi_{c}(2470) K, \Xi_{c}^{\prime}(2575) K$, and $\Xi_{c}^{\prime *}(2645) K$ modes are predicted to be

$$
\frac{\Gamma\left[\Xi_{c} K, \Xi_{c}^{\prime} K, \Xi_{c}^{*} K\right]}{\Gamma_{\text {total }}} \simeq 71 \%, 21 \%, 7 \%
$$

From Fig. 7, it is found that the strong decay properties of $\left|\Omega_{c}{ }^{4} D_{\lambda \lambda^{2}}{ }^{+}\right\rangle$are less sensitive to its mass. The $\Xi_{c}(2470) K$ and $\Xi_{c}^{\prime}(2575) K$ may be optimal channels for us to search for this missing $J^{P}=1 / 2^{+}$state $\left|\Omega_{c}{ }^{4} D_{\lambda \lambda \frac{1}{2}}{ }^{+}\right\rangle$.

We also estimate its radiative decays into the $1 P$-wave charmed baryon states. Our results are listed in Table XII. It is found that $\left|\Omega_{c}^{04} D_{\lambda \lambda}{ }^{1}{ }^{+}\right\rangle$might have relatively large decay rates into $\left|\Omega_{c}^{04} P_{\lambda^{2}}{ }^{-}\right\rangle \gamma$ and $\left|\Omega_{c}^{04} P_{\lambda^{\frac{3}{2}}}{ }^{-}\right\rangle \gamma$, and the partial radiative decay width is estimated to be $\mathcal{O}(10) \mathrm{keV}$. The branching fractions for these main radiative decay processes may be $\mathcal{O}\left(10^{-3}\right)$.

\section{2. $J^{P}=3 / 2^{+}$states}

For the $J^{P}=3 / 2^{+}$state $\left|\Omega_{c}{ }^{2} D_{\lambda \lambda}{ }^{\frac{3}{2}}{ }^{+}\right\rangle$, the width is predicted to be $\Gamma_{\text {total }} \sim 18 \mathrm{MeV}$, which is less sensitive to the phase space of strong decays (see Fig. 7). It is found that $\Xi_{c}(2470) K$ together with $\Xi_{c}^{\prime}(2575) K$ governs the decays of $\left|\Omega_{c}{ }^{2} D_{\lambda \lambda \frac{3}{2}}{ }^{+}\right\rangle$. Their branching fractions are predicted to be

TABLE XII. Partial widths of strong and radiative decays for the $\lambda$-mode $D$-wave $\Omega_{c}$ and $\Omega_{b}$ baryons, the masses (MeV) of which are taken from the quark model predictions of Ref. [21]. $M_{f}$ stands for the masses of $P$-wave heavy baryons $(\mathrm{MeV})$ in the final states, which are adopted from the RPP [24] and Refs. [1,21]. The units for the partial widths of radiative and strong decays are keV and MeV, respectively. The superscript (subscript) stands for the uncertainty of a prediction with a $+10 \%(-10 \%)$ uncertainty of the oscillator parameter $\alpha_{\rho}$.

\begin{tabular}{|c|c|c|c|c|c|c|c|c|c|c|c|c|c|}
\hline \multirow[b]{2}{*}{ Decay mod } & \multirow[b]{2}{*}{$M_{f}$} & \multicolumn{2}{|c|}{$\left|\Omega_{c}^{2} D_{\lambda \lambda^{2}}{ }^{+}\right\rangle(3282)$} & \multicolumn{2}{|c|}{$\left|\Omega_{c}^{2} D_{\lambda \lambda \frac{2}{2}}{ }^{+}\right\rangle(3286)$} & \multicolumn{2}{|c|}{$\left|\Omega_{c}{ }^{4} D_{\lambda \lambda \frac{1}{2}}{ }^{+}\right\rangle(3287)$} & \multicolumn{2}{|c|}{$\left|\Omega_{c}{ }^{4} D_{\lambda \lambda^{\frac{3}{2}}}{ }^{+}\right\rangle(3298)$} & \multicolumn{2}{|c|}{$\left|\Omega_{c}{ }^{4} D_{\lambda \lambda \frac{5}{2}}{ }^{+}\right\rangle(3297)$} & \multicolumn{2}{|c|}{$\left|\Omega_{c}{ }^{4} D_{\lambda \lambda^{\frac{7}{2}}}{ }^{+}\right\rangle(3283)$} \\
\hline & & $\Gamma_{i}$ & $\mathcal{B}_{i}(\%)$ & $\Gamma_{i}$ & $\mathcal{B}_{i}(\%)$ & $\Gamma_{i}$ & $\mathcal{B}_{i}(\%)$ & $\Gamma$ & $\overline{\mathcal{B}_{i}(\%)}$ & $\Gamma_{i}$ & $\mathcal{B}_{i}(\%)$ & $\Gamma_{i}$ & $\mathcal{B}_{i}(\%)$ \\
\hline$\Xi_{c} K$ & 2470 & $7.97_{-3.61}^{+3.85}$ & 44.7 & $5.16_{+142}^{-1.13}$ & 57.3 & $15.9_{-72}^{+7.8}$ & 71.4 & $7.90_{-3.33}^{+4.08}$ & 48.2 & $1.65_{+0.44}^{-0.35}$ & 19.8 & $6.43_{+1.77}^{-1.43}$ & 81.7 \\
\hline${ }_{c}^{\prime} K$ & 2575 & $9.26_{-255}^{+3.31}$ & 51.9 & $\begin{array}{l}1.77_{++064}^{-0.42} \\
1.45\end{array}$ & 19.7 & $4.71_{-1.34}^{+1.22}$ & 20.8 & $\begin{array}{l}1.0-3.73 \\
2.44_{-073}^{+0.68}\end{array}$ & 14.8 & $0.15_{+0.05}^{-0.44}$ & 1.79 & $0.54_{+2020}^{-0.14}$ & 6.73 \\
\hline$\Xi_{c}^{* *} K$ & 2645 & $0.50_{+0.02}^{-0.01}$ & 2.8 & $2.05_{-0.32}^{+0.04}$ & 22.8 & $1.65_{-0.31}^{+0.25}$ & 7.4 & $5.97_{-1.03}^{+0.90}$ & 36.4 & $6.61_{+0.64}^{-0.00}$ & 79.2 & $0.88_{-0.12}^{+0.11}$ & 11.2 \\
\hline$\left|\Omega_{c} 1^{2} P_{\lambda 2^{1}}{ }^{-}\right\rangle \gamma$ & 3000 & $91.8_{+18.8}^{-0.02}$ & 0.51 & $9.79_{+2.12}^{-1.32}$ & 0.11 & 0.03 & $<0.01$ & $0.49_{+0.06}^{-0.03}$ & $<0.01$ & $0.19_{+0.02}^{-0.02}$ & $<0.01$ & $\simeq 0.0$ & $<0.01$ \\
\hline$\left|\Omega_{c} 1^{2} P_{\lambda{ }^{2}}{ }^{-}\right\rangle \gamma$ & 3066 & $18.4_{+4.0}^{-3.0}$ & 0.10 & $39.7_{+8.9}^{+6.12}$ & 0.44 & 0.04 & $<0.01$ & 0.02 & $<0.01$ & $0.17_{+0.02}^{-0.02}$ & $<0.01$ & $0.13_{+0.02}^{-0.02}$ & $<0.01$ \\
\hline$\left|\Omega_{c} 1^{4} P_{\lambda{ }^{1}}^{\frac{1}{2}}\right\rangle \gamma$ & 3050 & $0.13_{+0.01}^{-0.02}$ & $<0.01$ & 0.008 & $<0.01$ & $70.3_{+14.8}^{-11.3}$ & 0.32 & $34.7_{+73}^{-5.6}$ & 0.21 & $5.56_{+120}^{-0.91}$ & 0.07 & $1.07_{+0.30}^{-0.21}$ & 0.01 \\
\hline$\left|\Omega_{c} 1^{4} P_{\lambda \lambda^{\frac{3}{2}}}\right\rangle \gamma$ & 3050 & $0.2_{+0.03}^{-0.03}$ & $<0.01$ & 0.09 & $<0.01$ & $27.5_{+5.9}^{+4.5}$ & 0.12 & $54.1_{+11.4}^{+8.3}$ & 0.33 & $39.8_{+8.6}^{-6.6}$ & 0.47 & $5.62_{+.9 .23}^{-0.090}$ & 0.07 \\
\hline$\left|\Omega_{c} 1^{4} P_{\lambda \frac{5}{2}}\right\rangle \gamma$ & 3090 & 0.03 & $<0.01$ & $0.14_{+0.03}^{-0.02}$ & $<0.01$ & $2.44_{+0.62}^{-0.45}$ & $<0.10$ & $7.95_{+1.82}^{-1.46}$ & 0.05 & $19.6_{+4.3}^{-3.0}$ & 0.24 & $23.1_{+5.3}^{+3.99}$ & 0.29 \\
\hline \multirow[t]{2}{*}{ Sum } & \multicolumn{3}{|c|}{$17.84_{-6.16}^{+6.16}$} & \multicolumn{2}{|l|}{$9.03_{+1.73}^{-1.30}$} & \multicolumn{2}{|c|}{$22.33_{-8.83}^{+9.25}$} & \multicolumn{2}{|l|}{$16.21_{-5.47}^{+5.64}$} & \multicolumn{2}{|l|}{$8.34_{+1.11}^{-0.98}$} & \multicolumn{2}{|l|}{$7.82_{+1.85}^{-1.46}$} \\
\hline & & \multicolumn{2}{|c|}{$\left|\Omega_{b}^{2} D_{\lambda \lambda^{3}}{ }^{+}\right\rangle(6530)$} & \multicolumn{2}{|c|}{$\left|\Omega_{b}^{2} D_{\lambda \lambda \frac{5}{2}}{ }^{+}\right\rangle(6520)$} & \multicolumn{2}{|c|}{$\left|\Omega_{b}{ }^{4} D_{\lambda \lambda \frac{1}{2}}{ }^{+}\right\rangle(6540)$} & \multicolumn{2}{|c|}{$\mid \Omega_{b}{ }^{4} D_{\left.\lambda \lambda^{\frac{3}{2}}{ }^{+}\right\rangle(6549)}$} & \multicolumn{2}{|c|}{$\left|\Omega_{b}{ }^{4} D_{\lambda \lambda^{2}}{ }^{+}\right\rangle(6529)$} & \multicolumn{2}{|c|}{$\left|\Omega_{b}{ }^{4} D_{\lambda \lambda}{ }^{7} \frac{7}{}+\right\rangle(6517$} \\
\hline Decay mode & $M_{f}$ & $\Gamma_{i}$ & $\mathcal{B}_{i}(\%)$ & $\Gamma_{i}$ & $\mathcal{B}_{i}(\%)$ & $\Gamma_{i}$ & $\mathcal{B}_{i}(\%)$ & $\Gamma$ & $\mathcal{B}_{i}(\%)$ & $\Gamma_{i}$ & $\mathcal{B}_{i}(\%)$ & $\Gamma_{i}$ & $\mathcal{B}_{i}(\%)$ \\
\hline${ }_{b} K$ & 5795 & $11.2_{-5.1}^{+5.5}$ & 55.5 & $6.18_{+1.71}^{-1.36}$ & 75.83 & $22.1_{-10.5}^{+11.7}$ & 76.98 & $10.9_{-5.4}^{+6.1}$ & 53.48 & $2.07_{+0.48}^{-0.49}$ & 28.09 & $7.83_{+2.13}^{-1.69}$ & 90.84 \\
\hline$\Xi_{b}^{\prime} K$ & 5935 & $8.43_{-1.38}^{+1.1}$ & 41.8 & $0.30_{+0.13}^{-0.09}$ & 3.68 & $4.69_{-0.86}^{+0.73}$ & 16.34 & $2.55_{-0.51}^{+5.44}$ & 12.51 & 0.03 & 0.41 & 0.09 & 1.04 \\
\hline$\Xi_{b}^{\prime *} K$ & 5955 & $0.51_{-0.10}^{-0.09}$ & 2.52 & $1.65_{-016}^{+0.15}$ & 20.25 & $1.86_{-0.27}^{-0.02}$ & 6.48 & $6.87_{-1.01}^{+1.81}$ & 33.71 & $5.24_{-0.43}^{+0.37}$ & 71.10 & $0.68_{-0.07}^{+0.06}$ & 7.89 \\
\hline$\left|\Omega_{b} 1^{2} P_{\lambda \frac{1}{2}-}\right\rangle \gamma$ & 6301 & $33.2_{+6.5}^{-5.1}$ & 0.16 & $4.03_{+0.86}^{-0.10}$ & 0.05 & $0.55_{+0.10}^{-0.08}$ & $<0.01$ & $1.20_{+0.19}^{-0.16}$ & $<0.01$ & $0.32_{+0.05}^{-0.05}$ & $<0.01$ & $\simeq 0.0$ & $<0.01$ \\
\hline$\left|\Omega_{b} 1^{2} P_{\lambda 2^{\frac{3}{2}}}\right\rangle \gamma$ & 6304 & $17.9_{+3.7}^{-2.3}$ & 0.09 & $16.8_{+3.6}^{-2.86}$ & 0.21 & $0.26_{+0.05}^{-0.04}$ & $<0.01$ & $0.10_{+0.02}^{-0.19}$ & $<0.01$ & $0.73_{+0.12}^{-0.10}$ & $<0.01$ & $0.56_{+0.09}^{-0.07}$ & $<0.01$ \\
\hline$\left|\Omega_{b} 1^{4} P_{\lambda \lambda^{2}}\right\rangle \gamma$ & 6312 & $0.47_{+0.07}^{-0.7}$ & $<0.01$ & 0.024 & $<0.01$ & $40.4_{+7.5}^{-6.0 .1}$ & 0.14 & $18.8_{+3.5}^{+2.02}$ & 0.09 & $2.72_{+0.57}^{+0.12}$ & 0.04 & $0.50_{++.15}^{-0.09}$ & $<0.01$ \\
\hline$\left|\Omega_{b} 1^{4} P_{\lambda^{2}}^{\frac{3}{2}}\right\rangle \gamma$ & 6311 & $0.74_{+0.13}^{-0.10}$ & $<0.01$ & $0.27_{+0.04}^{-0.04}$ & $<0.01$ & $17.9_{+3.5}^{+2.8}$ & 0.06 & $31.0_{+5.9}^{+4.7}$ & 0.15 & $15.4_{+3.2}^{-2.5}$ & 0.21 & $2.70_{+0.58}^{-0.44}$ & 0.03 \\
\hline$\left|\Omega_{b} 1^{4} P_{\lambda^{2}}{ }^{\frac{5}{2}}\right\rangle \gamma$ & & $0.21_{+0.04}^{-0.03}$ & $<0.01$ & $0.91_{+0.17}^{-0.04}$ & $<0.01$ & $2.83_{+0.81}^{-0.56}$ & 0.01 & $8.98_{+1.98}^{-1.50}$ & 0.04 & $14.6_{+2.0}^{-3.3 .3}$ & 0.20 & $12.1_{+2.6}^{-2.0}$ & 0.14 \\
\hline Sum & & $20.19_{-6.57}^{+6.57}$ & & $8.15_{+1.68}^{-1.30}$ & & $28.71_{-11.61}^{+12.65}$ & & $20.38_{-6.91}^{+8.38}$ & & $7.37_{+0.04}^{-0.11}$ & & $8.62_{+2.06}^{-1.63}$ & \\
\hline
\end{tabular}




$$
\frac{\Gamma\left[\Xi_{c} K, \Xi_{c}^{\prime} K\right]}{\Gamma_{\text {total }}} \simeq 45 \%, 52 \% .
$$

The $\Xi_{c}(2470) K$ and $\Xi_{c}^{\prime}(2575) K$ may be ideal channels for us to search for this missing $J^{P}=3 / 2^{+}$state $\left|\Omega_{c}{ }^{2} D_{\lambda \lambda^{2}}{ }^{+}\right\rangle$.

For the other $J^{P}=3 / 2^{+}$state $\left|\Omega_{c}{ }^{4} D_{\lambda \lambda^{2}}{ }^{+}\right\rangle$, if its mass is below the threshold of $\Xi_{c}(2790) K$, the width is predicted to be $\Gamma_{\text {total }} \sim 16 \mathrm{MeV}$, which is less sensitive to the phase space of strong decays (see Fig. 7). In this case, both $\Xi_{c}(2470) K$ and $\Xi_{c}^{\prime *}(2645) K$ are the dominant decay channels of $\left|\Omega_{c}{ }^{2} D_{\lambda \lambda^{2}}{ }^{3}\right\rangle$, while the decay rate into $\Xi_{c}^{\prime}(2575) K$ is sizeable as well. Their branching fractions are predicted to be

$$
\frac{\Gamma\left[\Xi_{c} K, \Xi_{c}^{\prime *} K, \Xi_{c}^{\prime} K\right]}{\Gamma_{\text {total }}} \simeq 48 \%, 36 \%, 15 \% .
$$

However, if the $\Xi_{c}(2790) K$ decay channel is open, this decay mode should be the dominant decay mode. The decay width of $\left|\Omega_{c}{ }^{4} D_{\lambda \lambda^{2}}{ }^{3}\right\rangle$ can reach up to $30-40 \mathrm{MeV}$. The $\Xi_{c}(2470) K$ and $\Xi_{c}^{\prime *}(2645) K$ may be ideal channels for us to search for this missing $J^{P}=3 / 2^{+}$state $\mid \Omega_{c}{ }^{4} D_{\lambda \lambda \lambda^{\frac{3}{2}}}{ }^{+}$.

We also estimate the radiative decays of these $J^{P}=$ $3 / 2^{+} \Omega_{c}$ states into the $1 P$-wave charmed baryon states. Our results are listed in Table XII. It is found that $\left|\Omega_{c}^{02} D_{\lambda \lambda^{\frac{3}{2}}}{ }^{+}\right\rangle \rightarrow\left|\Omega_{c}^{02} P_{\lambda \frac{1}{2}^{-}}\right\rangle \gamma$ and $\left|\Omega_{c}^{04} D_{\lambda \lambda^{\frac{3}{2}}}{ }^{+}\right\rangle \rightarrow\left|\Omega_{c}^{04} P_{\lambda{ }^{\frac{1}{2}}}{ }^{-}\right\rangle \gamma$, $\left|\Omega_{c}^{04} P_{\lambda^{2}}{ }^{-}\right\rangle \gamma$ might have relatively large decay rates. The partial radiative decay widths are estimated to be $\mathcal{O}(10) \mathrm{keV}$, while their branching fractions may reach up to $\mathcal{O}\left(10^{-3}\right)$.

\section{3. $J^{P}=5 / 2^{+}$states}

For the $J^{P}=5 / 2^{+}$state $\left|\Omega_{c}{ }^{2} D_{\lambda \lambda^{2}}{ }^{+}\right\rangle$, if we take its mass as the prediction $3286 \mathrm{MeV}$ in Ref. [21], the width is predicted to be $\Gamma_{\text {total }} \sim 9 \mathrm{MeV}$, which is slightly dependent on the phase space of strong decays (see Fig. 7). The decays of $\left|\Omega_{c}{ }^{2} D_{\lambda \lambda^{\frac{3}{2}}}{ }^{+}\right\rangle$are governed by the $\Xi_{c}(2470) K$ channel, while the decay rates into $\Xi_{c}^{\prime}(2575) K$ and $\Xi_{c}^{\prime *}(2645) K$ are sizeable as well. Their branching fractions are predicted to be

$$
\frac{\Gamma\left[\Xi_{c} K, \Xi_{c}^{\prime *} K, \Xi_{c}^{\prime} K\right]}{\Gamma_{\text {total }}} \simeq 57 \%, 23 \%, 20 \% .
$$

The $\Xi_{c}(2470) K, \Xi_{c}^{\prime}(2575) K$, and $\Xi_{c}^{\prime *}(2645) K$ may be ideal channels for us to search for this missing $J^{P}=5 / 2^{+}$ state $\left|\Omega_{c}^{2} D_{\lambda \lambda \frac{5}{2}}{ }^{+}\right\rangle$.

For the other $J^{P}=5 / 2^{+}$state $\left|\Omega_{c}{ }^{4} D_{\lambda \lambda \frac{5}{2}}{ }^{+}\right\rangle$, if we take its mass as the prediction $3297 \mathrm{MeV}$ in Ref. [21], the width is predicted to be $\Gamma_{\text {total }} \sim 8 \mathrm{MeV}$, which is slightly dependent on the phase space of strong decays (see Fig. 7). The $\Xi_{c}^{* *}(2645) K$ are the dominant decay channels of $\left|\Omega_{c}{ }^{2} D_{\lambda \lambda^{2}}{ }^{+}\right\rangle$, while the decay rate into $\Xi_{c}(2470) K$ is sizeable as well. Their branching fractions are predicted to be

$$
\frac{\Gamma\left[\Xi_{c}^{\prime *} K, \Xi_{c} K\right]}{\Gamma_{\text {total }}} \simeq 78 \%, 19 \% .
$$

The $\Xi_{c}(2470) K$ and $\Xi_{c}^{\prime *}(2645) K$ may be ideal channels for us to search for this missing $J^{P}=5 / 2^{+}$state $\left|\Omega_{c}{ }^{4} D_{\lambda \lambda^{2}}{ }^{5}\right\rangle$.

We also estimate the radiative decays of the $J^{P}=5 / 2^{+}$ $\Omega_{c}$ states into the $1 P$-wave charmed baryon states. Our results are listed in Table XII. It is found that $\left|\Omega_{c}^{02} D_{\lambda \lambda \frac{5}{2}}{ }^{+}\right\rangle \rightarrow$ $\left|\Omega_{c}^{02} P_{\lambda{ }^{3}}{ }^{-}\right\rangle \gamma$ and $\left|\Omega_{c}^{04} D_{\lambda \lambda^{2}}{ }^{+}\right\rangle \rightarrow\left|\Omega_{c}^{04} P_{\lambda^{3}}{ }^{-}\right\rangle \gamma$ might have relatively large decay rates. The partial radiative decay widths are estimated to be $\mathcal{O}(10) \mathrm{keV}$, while their branching fractions may reach up to $\mathcal{O}\left(10^{-3}\right)$.

$$
\text { 4. } J^{P}=7 / 2^{+} \text {state }
$$

The $J^{P}=7 / 2^{+}$state $\left|\Omega_{c}{ }^{4} D_{\lambda \lambda^{7}}{ }^{7+}\right\rangle$ may be a narrow state with a width of a few $\mathrm{MeV}$. If its mass is taken to be $3283 \mathrm{MeV}$ as predicted in Ref. [21], the width is predicted to be $\Gamma_{\text {total }} \sim 8 \mathrm{MeV}$, which shows some sensitivities to the phase space of strong decays (see Fig. 7). The decays of $\left|\Omega_{c}{ }^{4} D_{\lambda \lambda^{\frac{7}{2}}}{ }^{+}\right\rangle$are governed by the $\Xi_{c}(2470) K$ channel. The branching fraction is predicted to be

$$
\frac{\Gamma\left[\Xi_{c} K\right]}{\Gamma_{\text {total }}} \simeq 80 \% \text {. }
$$

The $\Xi_{c}(2470) K$ may be an ideal channel for our search for

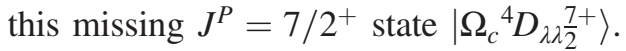

We also estimate its radiative decays into the $1 P$-wave charmed baryon states. Our results are listed in Table XII. It is found that $\left|\Omega_{c}^{04} D_{\lambda \lambda \overline{2}^{7}}\right\rangle$ might have a relatively large decay rate into $\left|\Omega_{c}^{04} P_{\lambda \frac{5}{2}}{ }^{-}\right\rangle \gamma$, and the partial radiative decay width is estimated to be $\mathcal{O}(10) \mathrm{keV}$. The branching fraction for this main radiative decay process may be $\mathcal{O}\left(10^{-3}\right)$.

\section{F. $\Omega_{b}$}

In the $\Omega_{b}$ family, according to the quark model classification, there are six $\lambda$-mode $1 D$-wave excitations: $\left|\Omega_{b}{ }^{4} D_{\lambda \lambda \frac{1}{2}}{ }^{+}\right\rangle,\left|\Omega_{b}{ }^{4} D_{\lambda \lambda^{\frac{3}{2}}}{ }^{+}\right\rangle,\left|\Omega_{b}{ }^{2} D_{\lambda \lambda^{2}}{ }^{+}\right\rangle,\left|\Omega_{b}{ }^{2} D_{\lambda \lambda^{2}}{ }^{\frac{5}{}}\right\rangle$, $\left|\Omega_{b}{ }^{4} D_{\lambda \lambda^{2}}{ }^{+}\right\rangle$, and $\left|\Omega_{b}{ }^{4} D_{\lambda \lambda^{2}}{ }^{+}\right\rangle$. However, no $D$-wave states have been established. The typical masses of the $\lambda$-mode $1 D$-wave $\Omega_{b}$ excitations are $\sim 6.6 \mathrm{GeV}$ within various quark model predictions (see Table II). In these possible mass ranges, we study their strong decay transitions by emitting one light pseudoscalar meson within the ChQM. Our results are shown in Fig. 7. To be more specific, taking the masses of the $1 D$-wave states obtained in the relativistic quark-diquark picture [21], we give the predicted widths in Table XII. 


\section{1. $J^{P}=1 / 2^{+}$state}

The $J^{P}=1 / 2^{+}$state $\left|\Omega_{b}{ }^{4} D_{\lambda \lambda^{2}}{ }^{1+}\right\rangle$ may be a narrow state with a width of $\Gamma_{\text {total }} \sim 30 \mathrm{MeV}$. Its decays are dominated by the $\Xi_{b}(5795) K$. The decay rates into $\Xi_{b}^{\prime}(5935) K$ and $\Xi_{b}^{\prime *}(5955) K$ are sizeable as well. The branching fractions for the $\Xi_{b}(5795) K, \Xi_{b}^{\prime}(5935) K$, and $\Xi_{b}^{\prime *}(5955) K$ modes are predicted to be

$$
\frac{\Gamma\left[\Xi_{b} K, \Xi_{b}^{\prime} K, \Xi_{b}^{\prime *} K\right]}{\Gamma_{\text {total }}} \simeq 76 \%, 16 \%, 7 \% .
$$

From Fig. 7, it is found that the strong decay properties of $\left|\Omega_{b}{ }^{4} D_{\lambda \lambda^{2}}{ }^{+}\right\rangle$are less sensitive to its mass. $\Xi_{b}(5795) K$ and $\Xi_{b}^{\prime}(5935) K$ may be ideal channels for us to search for this missing $J^{P}=1 / 2^{+}$state $\left|\Omega_{b}{ }^{4} D_{\lambda \lambda^{2}}{ }^{+}\right\rangle$.

We also estimate its radiative decays into the $1 P$-wave bottom baryon states. Our results are listed in Table XII. It is found that $\left|\Omega_{b}^{-4} D_{\lambda \lambda 1^{2}}\right\rangle$ might have a relatively large decay rate into $\left|\Omega_{b}^{-4} P_{\lambda}^{1-}{ }^{-}\right\rangle \gamma$. The partial radiative decay width of this process is estimated to be $\mathcal{O}(10) \mathrm{keV}$, while the branching fraction may be $\mathcal{O}\left(10^{-3}\right)$.

\section{2. $J^{P}=3 / 2^{+}$states}

For the $J^{P}=3 / 2^{+}$state $\left|\Omega_{b}^{2} D_{\lambda \lambda^{2}}{ }^{+}\right\rangle$, the width is predicted to be $\Gamma_{\text {total }} \sim 20 \pm 5 \mathrm{MeV}$, which is slightly dependent on the phase space of strong decays (see Fig. 7). It is found that $\Xi_{b}(5795) K$ together with $\Xi_{b}^{\prime}(5935) K$ governs the decays of $\left|\Omega_{b}{ }^{2} D_{\lambda \lambda^{2}}{ }^{+}\right\rangle$. If its mass is taken to be $6530 \mathrm{MeV}$ as predicted in Ref. [21], the branching fractions for the $\Xi_{b}(5795) K$ and $\Xi_{b}^{\prime}(5935) K$ modes are predicted to be

$$
\frac{\Gamma\left[\Xi_{b} K, \Xi_{b}^{\prime} K\right]}{\Gamma_{\text {total }}} \simeq 55 \%, 42 \%,
$$

The $\Xi_{b}(5795) K$ and $\Xi_{b}^{\prime}(5935) K$ may be ideal channels for us to search for this missing $J^{P}=3 / 2^{+}$state $\left|\Omega_{b}{ }^{2} D_{\lambda \lambda^{2}}{ }^{+}\right\rangle$.

For the other $J^{P}=3 / 2^{+}$state $\left|\Omega_{b}{ }^{4} D_{\lambda \lambda^{2}}{ }^{+}\right\rangle$, if its mass is taken to be $6549 \mathrm{MeV}$ as predicted in Ref. [21], the width is predicted to be $\Gamma_{\text {total }} \sim 20 \mathrm{MeV}$. The decays are dominated by the $\Xi_{b}(5795) K$ and $\Xi_{b}^{\prime *}(5955) K$ channels, while the decay rate into $\Xi_{b}^{\prime}(5935) K$ is sizeable as well. Their branching fractions are predicted to be

$$
\frac{\Gamma\left[\Xi_{b} K, \Xi_{b}^{\prime *} K, \Xi_{b}^{\prime} K\right]}{\Gamma_{\text {total }}} \simeq 53 \%, 34 \%, 12 \%,
$$

which is sensitive to the phase space of strong decays (see Fig. 7). $\Xi_{b}(5795) K$ and $\Xi_{b}^{\prime *}(5955) K$ may be ideal channels for our search for this missing $J^{P}=3 / 2^{+}$state $\left|\Omega_{b}{ }^{4} D_{\lambda \lambda^{2}}{ }^{+}\right\rangle$.

We also estimate the radiative decays of these $J^{P}=$ $3 / 2^{+} \Omega_{b}$ states into the $1 P$-wave bottom baryon states. Our results are listed in Table XII. It is found that
$\left|\Omega_{b}^{-2} D_{\lambda \lambda^{\frac{3}{2}}}{ }^{+}\right\rangle \rightarrow\left|\Omega_{b}^{-2} P_{\lambda 2^{1}}{ }^{-}\right\rangle \gamma$ and $\left|\Omega_{b}^{-4} D_{\lambda \lambda^{\frac{3}{2}}}{ }^{+}\right\rangle \rightarrow\left|\Omega_{b}^{-4} P_{\lambda^{\frac{3}{2}}}{ }^{-}\right\rangle \gamma$ might have relatively large decay rates. The partial radiative decay widths are estimated to be $\mathcal{O}(10) \mathrm{keV}$, while their branching fractions may reach up to $\mathcal{O}\left(10^{-3}\right)$.

$$
\text { 3. } J^{P}=5 / 2^{+} \text {states }
$$

For the $J^{P}=5 / 2^{+}$state $\left|\Omega_{b}{ }^{2} D_{\lambda \lambda^{2}}{ }^{+}\right\rangle$, if we take its mass as the prediction $6520 \mathrm{MeV}$ in Ref. [21], the width is predicted to be $\Gamma_{\text {total }} \sim 8 \mathrm{MeV}$. The decays of $\left|\Omega_{b}^{2} D_{\lambda \lambda^{2}}{ }^{+}\right\rangle$ are governed by the $\Xi_{b}(5795) K$ channel, while the decay rate into $\Xi_{b}^{\prime *}(5955) K$ is sizeable as well. Their branching fractions are predicted to be

$$
\frac{\Gamma\left[\Xi_{b} K, \Xi_{b}^{\prime *} K\right]}{\Gamma_{\text {total }}} \simeq 76 \%, 20 \% .
$$

It should be pointed out that the decay properties of $\left|\Omega_{b}{ }^{2} D_{\lambda \lambda 2^{\frac{5}{}}}\right\rangle$ show some sensitivities to its mass (see Fig. 7). $\Xi_{b}(5795) K$ and $\Xi_{b}^{\prime *}(5955) K$ may be ideal channels for us to search for this missing $J^{P}=5 / 2^{+}$state $\left|\Omega_{b}^{2} D_{\lambda \lambda^{\frac{5}{2}}}{ }^{+}\right\rangle$.

For the other $J^{P}=5 / 2^{+}$state $\left|\Omega_{b}{ }^{4} D_{\lambda \lambda^{2}}{ }^{+}\right\rangle$, if we take its mass as the prediction $6549 \mathrm{MeV}$ in Ref. [21], the width is predicted to be $\Gamma_{\text {total }} \sim 8 \mathrm{MeV}$. $\Xi_{b}^{* *}(5955) K$ is the dominant decay channel of $\left|\Omega_{b}{ }^{4} D_{\lambda \lambda \frac{5}{2}}{ }^{+}\right\rangle$, while the decay rate into $\Xi_{b}(5790) K$ is sizeable as well. Their branching fractions are predicted to be

$$
\frac{\Gamma\left[\Xi_{b}^{\prime *} K, \Xi_{b} K\right]}{\Gamma_{\text {total }}} \simeq 74 \%, 25 \% .
$$

The decay properties of $\left|\Omega_{b}{ }^{4} D_{\lambda \lambda}{ }^{\frac{5}{2}}{ }^{+}\right\rangle$show some sensitivities to its mass (see Fig. 7). The $\Xi_{b}(5795) K$ and $\Xi_{b}^{* *}(5955) K$ may be ideal channels for us to search for this missing $J^{P}=5 / 2^{+}$state $\left|\Omega_{b}^{4} D_{\lambda \lambda^{2}}{ }^{+}\right\rangle$.

We also estimate the radiative decays of the $J^{P}=5 / 2^{+}$ $\Omega_{b}$ states into the $1 P$-wave bottom baryon states. Our results are listed in Table XII. It is found that $\left|\Omega_{b}^{-2} D_{\lambda \lambda} \lambda^{\frac{5}{2}}\right\rangle \rightarrow$ $\left|\Omega_{b}^{-2} P_{\lambda^{\frac{3}{2}}}{ }^{-}\right\rangle \gamma$ and $\left|\Omega_{b}^{-4} D_{\lambda \lambda^{2}}{ }^{+}\right\rangle \rightarrow\left|\Omega_{b}^{-4} P_{\lambda^{2}}{ }^{-}\right\rangle \gamma,\left|\Omega_{b}^{-4} P_{\lambda^{\frac{5}{2}}}^{-}\right\rangle \gamma$ might have relatively large decay rates. The partial radiative decay widths are estimated to be $\mathcal{O}(10) \mathrm{keV}$, while their branching fractions may reach up to $\mathcal{O}\left(10^{-3}\right)$.

$$
\text { 4. } J^{P}=7 / 2^{+} \text {state }
$$

The $J^{P}=7 / 2^{+}$state $\left|\Omega_{b}{ }^{4} D_{\lambda \lambda^{7}}{ }^{+}\right\rangle$may be a narrow state with a width of a few $\mathrm{MeV}$ or a few tens of $\mathrm{MeV}$. If its mass is taken to be $6517 \mathrm{MeV}$ as predicted in Ref. [21], the width is predicted to be $\Gamma_{\text {total }} \sim 8 \mathrm{MeV}$, which shows some sensitivities to the phase space of strong decays (see Fig. 7). The decays of $\left|\Omega_{b}{ }^{4} D_{\lambda \lambda}{ }^{\frac{7}{2}}\right\rangle$ are governed by the $\Xi_{b}(5970) K$ channel. The branching fraction is predicted to be 


$$
\frac{\Gamma\left[\Xi_{b} K\right]}{\Gamma_{\text {total }}} \simeq 89 \%
$$

$\Xi_{b}(5970) K$ may be an ideal channel for us to search for this missing $J^{P}=7 / 2^{+}$state $\left|\Omega_{b}{ }^{4} D_{\lambda \lambda^{2}}{ }^{7}\right\rangle$.

We also estimate its radiative decays into the $1 P$-wave bottom baryon states. Our results are listed in Table XII. It is found that $\left|\Omega_{b}^{-4} D_{\lambda \lambda^{2}}{ }^{+}\right\rangle$might have a relatively large decay rate into $\left|\Omega_{b}^{-4} P_{\lambda^{\frac{5}{2}}}\right\rangle \gamma$, and the partial radiative decay width is estimated to be $\mathcal{O}(10) \mathrm{keV}$. The branching fraction for this main radiative decay process may be $\mathcal{O}\left(10^{-3}\right)$.

\section{SUMMARY}

In this work, we carry out a systematic study of the strong decays with emission of one light pseudoscalar meson and the radiative decays with emission one photon of the low-lying $D$-wave singly heavy baryons in a constituent quark model. Our results may provide helpful references to establish these missing $D$-wave singly heavy baryons in future experiments. Several key results from this study can be learned as follows.

The $\lambda$-mode $1 D$-wave $J^{P}=3 / 2^{+}$and $J^{P}=5 / 2^{+}$excitations of $\overline{\mathbf{3}}_{F}$ (i.e., $\left|{ }^{2} D_{\lambda \lambda 2^{\frac{3}{2}}}{ }^{+}\right\rangle$and $\left|{ }^{2} D_{\lambda \lambda} \overline{2}^{+}\right\rangle$) in the $\Lambda_{c}$ and $\Xi_{c}$ families might have been observed in experiments. Both $\Lambda_{c}(2860)$ and $\Xi_{c}(3050)$ seem to favor the $J^{P}=3 / 2^{+}$ excitation $\left|{ }^{2} D_{\lambda \lambda^{\frac{3}{2}}}{ }^{+}\right\rangle$of $\overline{\mathbf{3}}_{F}$, while $\Lambda_{c}(2880)$ and $\Xi_{c}(3080)$ seem to favor assigning the $J^{P}=5 / 2^{+}$excitation $\left|{ }^{2} D_{\lambda \lambda^{2}}{ }^{+}\right\rangle$. The nature of $\Xi_{c}(3050)$ and $\Xi_{c}(3080)$ may be tested by the radiative transitions $\Xi_{c}(3055)^{0} \rightarrow \Xi_{c}(2790)^{0} \gamma$ and $\Xi_{c}(3080)^{0} \rightarrow \Xi_{c}(2815)^{0} \gamma$, respectively.

The missing $\lambda$-mode $1 D$-wave $J^{P}=3 / 2^{+}$and $J^{P}=$ $5 / 2^{+}$excitations of $\overline{\mathbf{3}}_{F}$ in the $\Lambda_{b}$ and $\Xi_{b}$ families have a large potential to be found in forthcoming experiments. They might be narrow states with a width of $\sim 10 \mathrm{MeV}$. In the $\Lambda_{b}$ family, the $J^{P}=3 / 2^{+}$state might be established in the $\Sigma_{b} \pi$ and $\Lambda_{b}(5912) \gamma$ final states, while the $J^{P}=5 / 2^{+}$ state might be established in the $\Sigma_{b}^{*} \pi$ and $\Lambda_{b}(5920) \gamma$ final states. In the $\Xi_{b}$ family, the $J^{P}=3 / 2^{+}$state might be established in the $\Xi_{b}^{\prime} \pi, \Xi_{b}^{\prime *} \pi$ and $\Sigma_{b} K$ final states, while the $J^{P}=5 / 2^{+}$state might be established in the $\Sigma_{b}^{*} K$ and $\Xi_{b}^{\prime *} \pi$ final states.
The $\lambda$-mode $1 D$-wave excitations of $\mathbf{6}_{F}$ in the $\Omega_{c}$ and $\Omega_{b}$ families have a large potential to be found in forthcoming experiments as well. They are fairly narrow states with a width of a few $\mathrm{MeV}$ or a few tens of $\mathrm{MeV}$. The kaonic decay channels $\Xi_{c}(2470) K, \Xi_{c}^{\prime}(2575) K$, and $\Xi_{c}^{\prime *}(2645) K$ may be ideal channels for us to search for these missing $1 D$-wave excited $\Omega_{c}$ states, while the kaonic decay channels $\Xi_{b} K$, $\Xi_{b}^{\prime} K$, and $\Xi_{b}^{\prime *} K$ may be ideal channels for us to search for these missing $1 D$-wave excited $\Omega_{b}$ states.

The $\lambda$-mode $1 D$-wave excitations in the $\Sigma_{c(b)}$ and $\Xi_{c(b)}^{\prime}$ families appear to have relatively broad widths. The sum of the partial widths with emission of a one-pion meson and one-kaon meson is about $50-200 \mathrm{MeV}$. These $1 D$-wave states might have large decay rates into the $1 P$-wave heavy baryon states via the pionic and/or kaonic decays. The $\Lambda_{c}(2595) \pi$ and $\Lambda_{c}(2625) \pi$ channels may be ideal channels for looking for the missing $1 D$-wave excitations in the $\Sigma_{c}$ family. The $\Xi_{c}(2790) \pi, \Xi_{c}(2815) \pi, \Lambda_{c}(2595) K$, and $\Lambda_{c}(2625) K$ decay channels may be ideal channels for looking for the missing $1 D$-wave excitations in the $\Xi_{c}^{\prime}$ family. The $\Lambda_{b}(5912) \pi$, and $\Lambda_{b}(5920) \pi$ decay channels may be ideal channels for looking for the missing $D$-wave excitations in the $\Sigma_{b}$ family.

Finally, it should be pointed out that some of our predictions bear a fairly large uncertainty from the nonrelativistic harmonic oscillator wave functions adopted in the calculations. Considering a $10 \%$ uncertainty of the oscillator parameter, one finds that the uncertainty of our predictions can reach up to $\sim 30 \%$. In some senses, our results are only a semiquantitative estimation based on the SU(3) symmetry. Fortunately, it is found that most of the featured results of the singly heavy baryons predicted in the present work and previous work [11] are consistent with other model approaches and observations, which indicates that the constituent quark model can still serve as a useful tool for investigating the heavy baryon mesonic decays and radiative transitions.

\section{ACKNOWLEDGMENTS}

We thank Qiang Zhao for helpful discussions. This work is supported by the National Natural Science Foundation of China under Grants No. 11775078.
[1] R. Aaij et al. (LHCb Collaboration), Observation of Five New Narrow $\Omega_{c}^{0}$ States Decaying to $\Xi_{c}^{+} K^{-}$, Phys. Rev. Lett. 118, 182001 (2017).
[2] K. L. Wang, L. Y. Xiao, X. H. Zhong, and Q. Zhao, Understanding the newly observed $\Omega_{c}$ states through their decays, Phys. Rev. D 95, 116010 (2017). 
[3] M. Padmanath and N. Mathur, Quantum Numbers of Recently Discovered $\Omega_{c}^{0}$ Baryons from Lattice QCD, Phys. Rev. Lett. 119, 042001 (2017).

[4] H. Y. Cheng and C. W. Chiang, Quantum numbers of $\Omega_{c}$ states and other charmed baryons, Phys. Rev. D 95, 094018 (2017).

[5] W. Wang and R. L. Zhu, Interpretation of the newly observed $\Omega_{c}^{0}$ resonances, Phys. Rev. D 96, 014024 (2017).

[6] M. Karliner and J.L. Rosner, Very narrow excited $\Omega_{c}$ baryons, Phys. Rev. D 95, 114012 (2017).

[7] B. Chen and X. Liu, New $\Omega_{c}^{0}$ baryons discovered by LHCb as the members of $1 P$ and $2 S$ states, Phys. Rev. D 96, 094015 (2017).

[8] H. X. Chen, Q. Mao, W. Chen, A. Hosaka, X. Liu, and S. L. Zhu, Decay properties of $P$-wave charmed baryons from light-cone QCD sum rules, Phys. Rev. D 95, 094008 (2017).

[9] S. S. Agaev, K. Azizi, and H. Sundu, Interpretation of the new $\Omega_{c}^{0}$ states via their mass and width, Eur. Phys. J. C 77, 395 (2017).

[10] R. Aaij et al. (LHCb Collaboration), Observation of a New $\Xi_{b}^{-}$Resonance, Phys. Rev. Lett. 121, 072002 (2018).

[11] K. L. Wang, Y. X. Yao, X. H. Zhong, and Q. Zhao, Strong and radiative decays of the low-lying $S$ - and $P$-wave singly heavy baryons, Phys. Rev. D 96, 116016 (2017).

[12] B. Chen, K. W. Wei, X. Liu, and A. Zhang, The role of newly discovered $\Xi_{b}(6227)^{-}$for constructing excited bottom baryon family, Phys. Rev. D 98, 031502 (2018).

[13] Q. Mao, H. X. Chen, W. Chen, A. Hosaka, X. Liu, and S. L. Zhu, QCD sum rule calculation for P-wave bottom baryons, Phys. Rev. D 92, 114007 (2015).

[14] C. Garcia-Recio, J. Nieves, O. Romanets, L. L. Salcedo, and L. Tolos, Odd parity bottom-flavored baryon resonances, Phys. Rev. D 87, 034032 (2013).

[15] Z. G. Wang, Analysis of the $1 / 2^{-}$and $3 / 2^{-}$heavy and doubly heavy baryon states with QCD sum rules, Eur. Phys. J. A 47, 81 (2011).

[16] J. Vijande, A. Valcarce, T. F. Carames, and H. Garcilazo, Heavy hadron spectroscopy: A quark model perspective, Int. J. Mod. Phys. E 22, 1330011 (2013).

[17] K. Thakkar, Z. Shah, A. K. Rai, and P. C. Vinodkumar, Excited state mass spectra and Regge trajectories of bottom baryons, Nucl. Phys. A965, 57 (2017).

[18] A. Valcarce, H. Garcilazo, and J. Vijande, Towards an understanding of heavy baryon spectroscopy, Eur. Phys. J. A 37, 217 (2008).

[19] M. Karliner, B. Keren-Zur, H. J. Lipkin, and J. L. Rosner, The quark model and $b$ baryons, Ann. Phys. (Amsterdam) 324, 2 (2009).

[20] B. Chen, K. W. Wei, and A. Zhang, Assignments of $\Lambda_{Q}$ and $\Xi_{Q}$ baryons in the heavy quark-light diquark picture, Eur. Phys. J. A 51, 82 (2015).

[21] D. Ebert, R. N. Faustov, and V. O. Galkin, Spectroscopy and Regge trajectories of heavy baryons in the relativistic quark-diquark picture, Phys. Rev. D 84, 014025 (2011).

[22] W. Roberts and M. Pervin, Heavy baryons in a quark model, Int. J. Mod. Phys. A 23, 2817 (2008).

[23] D. Ebert, R. N. Faustov, and V. O. Galkin, Masses of excited heavy baryons in the relativistic quark-diquark picture, Phys. Lett. B 659, 612 (2008).
[24] Particle Data Group, C. Patrignani et al., Review of particle physics, Chin. Phys. C 40, 100001 (2016).

[25] R. Aaij et al. (LHCb Collaboration), Study of the $D^{0} p$ amplitude in $\Lambda_{b}^{0} \rightarrow D^{0} p \pi^{-}$decays, J. High Energy Phys. 05 (2017) 030.

[26] D. Ebert, R. N. Faustov, and V. O. Galkin, Masses of heavy baryons in the relativistic quark model, Phys. Rev. D 72, 034026 (2005).

[27] T. Yoshida, E. Hiyama, A. Hosaka, M. Oka, and K. Sadato, Spectrum of heavy baryons in the quark model, Phys. Rev. D 92, 114029 (2015).

[28] K. Maltman and N. Isgur, Baryons with strangeness and charm in a quark model with chromodynamics, Phys. Rev. D 22, 1701 (1980).

[29] L. A. Copley, N. Isgur, and G. Karl, Charmed baryons in a quark model with hyperfine interactions, Phys. Rev. D 20, 768 (1979); Erratum, Phys. Rev. D23, 817(E) (1981).

[30] S. Capstick and N. Isgur, Baryons in a relativized quark model with chromodynamics, Phys. Rev. D 34, 2809 (1986).

[31] Z. Shah, K. Thakkar, A. K. Rai, and P. C. Vinodkumar, Excited state mass spectra of singly charmed baryons, Eur. Phys. J. A 52, 313 (2016).

[32] Z. Shah, K. Thakkar, A. K. Rai, and P. C. Vinodkumar, Mass spectra and Regge trajectories of $\Lambda_{c}^{+}, \Sigma_{c}^{0}, \Xi_{c}^{0}$ and $\Omega_{c}^{0}$ baryons, Chin. Phys. C 40, 123102 (2016).

[33] B. Chen, K. W. Wei, X. Liu, and T. Matsuki, Low-lying charmed and charmed-strange baryon states, Eur. Phys. J. C 77, 154 (2017).

[34] H. Garcilazo, J. Vijande, and A. Valcarce, Faddeev study of heavy baryon spectroscopy, J. Phys. G 34, 961 (2007).

[35] P. Pérez-Rubio, S. Collins, and G. S. Bali, Charmed baryon spectroscopy and light flavor symmetry from lattice QCD, Phys. Rev. D 92, 034504 (2015).

[36] M. Padmanath, R. G. Edwards, N. Mathur, and M. Peardon, Excited-state spectroscopy of singly, doubly and triply-charmed baryons from lattice QCD, arXiv:1311 .4806 .

[37] Q. Mao, H. X. Chen, A. Hosaka, X. Liu, and S. L. Zhu, $D$-wave heavy baryons of the $S U(3)$ flavor $\mathbf{6}_{F}$, Phys. Rev. D 96, 074021 (2017).

[38] H. X. Chen, Q. Mao, A. Hosaka, X. Liu, and S. L. Zhu, $D$-wave charmed and bottomed baryons from QCD sum rules, Phys. Rev. D 94, 114016 (2016).

[39] Z. G. Wang, Analysis of the $\Lambda_{c}(2860), \Lambda_{c}(2880)$, $\Xi_{c}(3055)$ and $\Xi_{c}(3080)$ as $D$-wave baryon states with QCD sum rules, Nucl. Phys. B926, 467 (2018).

[40] C. Chen, X. L. Chen, X. Liu, W. Z. Deng, and S. L. Zhu, Strong decays of charmed baryons, Phys. Rev. D 75, 094017 (2007).

[41] B. Chen, X. Liu, and A. Zhang, Newly observed $\Lambda_{c}(2860)^{+}$ at $\mathrm{LHCb}$ and its $D$-wave partners $\Lambda_{c}(2880)^{+}, \Xi_{c}(3055)^{+}$ and $\Xi_{c}(3080)^{+}$, Phys. Rev. D 95, 074022 (2017).

[42] Z. Zhao, D. D. Ye, and A. Zhang, Nature of charmed strange baryons $\Xi_{c}(3055)$ and $\Xi_{c}(3080)$, Phys. Rev. D 94, 114020 (2016).

[43] D. D. Ye, Z. Zhao, and A. Zhang, Study of $2 S$ - and $1 D$ excitations of observed charmed strange baryons, Phys. Rev. D 96, 114003 (2017). 
[44] H. Y. Cheng and C. K. Chua, Strong decays of charmed baryons in heavy hadron chiral perturbation theory, Phys. Rev. D 75, 014006 (2007).

[45] H. Y. Cheng and C. K. Chua, Strong decays of charmed baryons in heavy hadron chiral perturbation theory: An update, Phys. Rev. D 92, 074014 (2015).

[46] H. Y. Cheng, Charmed baryons circa 2015, Front. Phys. 10, 101406 (2015).

[47] X. H. Zhong and Q. Zhao, Charmed baryon strong decays in a chiral quark model, Phys. Rev. D 77, 074008 (2008).

[48] L. H. Liu, L. Y. Xiao, and X. H. Zhong, Charm-strange baryon strong decays in a chiral quark model, Phys. Rev. D 86, 034024 (2012).

[49] H. Nagahiro, S. Yasui, A. Hosaka, M. Oka, and H. Noumi, Structure of charmed baryons studied by pionic decays, Phys. Rev. D 95, 014023 (2017).

[50] S. Tawfiq, J. G. Korner, and P. J. O'Donnell, Electromagnetic transitions of heavy baryons in the $\mathrm{SU}\left(2 N_{f}\right) \times \mathrm{O}(3)$ symmetry, Phys. Rev. D 63, 034005 (2001).

[51] C. K. Chow, Radiative decays of excited $\Lambda_{Q}$ baryons in the bound state picture, Phys. Rev. D 54, 3374 (1996).

[52] D. Gamermann, C. E. Jimenez-Tejero, and A. Ramos, Radiative decays of dynamically generated charmed baryons, Phys. Rev. D 83, 074018 (2011).

[53] M. A. Ivanov, J. G. Korner, and V. E. Lyubovitskij, One photon transitions between heavy baryons in a relativistic three quark model, Phys. Lett. B 448, 143 (1999).

[54] H. Bahtiyar, K. U. Can, G. Erkol, M. Oka, and T. T. Takahashi, $\Xi_{c} \gamma \rightarrow \Xi_{c}^{\prime}$ transition in lattice QCD, Phys. Lett. B 772, 121 (2017).

[55] H. Bahtiyar, K. U. Can, G. Erkol, and M. Oka, $\Omega_{c} \gamma \rightarrow \Omega_{c}^{*}$ transition in lattice QCD, Phys. Lett. B 747, 281 (2015).

[56] S. L. Zhu and Y. B. Dai, Radiative decays of heavy hadrons from light cone QCD sum rules in the leading order of HQET, Phys. Rev. D 59, 114015 (1999).

[57] Z. G. Wang, Analysis of the vertexes $\Xi_{Q}^{*} \Xi_{Q}^{\prime} V, \Sigma_{Q}^{*} \Sigma_{Q} V$ and radiative decays $\Xi_{Q}^{*} \rightarrow \Xi_{Q}^{\prime} \gamma, \Sigma_{Q}^{*} \rightarrow \Sigma_{Q} \gamma$, Eur. Phys. J. A 44, 105 (2010).

[58] Z. G. Wang, Analysis of the vertexes $\Omega_{Q}^{*} \Omega_{Q} \phi$ and radiative decays $\Omega_{Q}^{*} \rightarrow \Omega_{Q} \gamma$, Phys. Rev. D 81, 036002 (2010).

[59] T. M. Aliev, K. Azizi, and H. Sundu, Radiative $\Omega_{Q}^{*} \rightarrow \Omega_{Q} \gamma$ and $\Xi_{Q}^{*} \rightarrow \Xi_{Q}^{\prime} \gamma$ transitions in light cone QCD, Eur. Phys. J. C 75, 14 (2015).

[60] T. M. Aliev, K. Azizi, and A. Ozpineci, Radiative decays of the heavy flavored baryons in light cone QCD sum rules, Phys. Rev. D 79, 056005 (2009).

[61] T. M. Aliev, T. Barakat, and M. Savc1, Analysis of the radiative decays $\Sigma_{Q} \rightarrow \Lambda_{Q} \gamma$ and $\Xi_{Q}^{\prime} \rightarrow \Xi_{Q} \gamma$ in light cone sum rules, Phys. Rev. D 93, 056007 (2016).

[62] M. J. Savage, E2 strength in the radiative charmed baryon decay $\Sigma_{c}^{*} \rightarrow \Lambda_{c} \gamma$, Phys. Lett. B 345, 61 (1995).

[63] M. C. Banuls, A. Pich, and I. Scimemi, Electromagnetic decays of heavy baryons, Phys. Rev. D 61, 094009 (2000).

[64] H. Y. Cheng, C. Y. Cheung, G. L. Lin, Y. C. Lin, T. M. Yan, and H. L. Yu, Chiral Lagrangians for radiative decays of heavy hadrons, Phys. Rev. D 47, 1030 (1993).

[65] N. Jiang, X. L. Chen, and S. L. Zhu, Electromagnetic decays of the charmed and bottom baryons in chiral perturbation theory, Phys. Rev. D 92, 054017 (2015).
[66] A. Bernotas and V. Šimonis, Radiative M1 transitions of heavy baryons in the bag model, Phys. Rev. D 87, 074016 (2013).

[67] T. M. Aliev, M. Savc1, and V. S. Zamiralov, Vector meson dominance and radiative decays of heavy spin-3/2 baryons to heavy spin-1/2 baryons, Mod. Phys. Lett. A 27, 1250054 (2012).

[68] J. Dey, V. Shevchenko, P. Volkovitsky, and M. Dey, Radiative decays of $S$ wave charmed baryons, Phys. Lett. B 337, 185 (1994).

[69] D. D. Ye, Z. Zhao, and A. Zhang, Study of $P$-wave excitations of observed charmed strange baryons, Phys. Rev. D 96, 114009 (2017).

[70] S.L. Zhu, Strong and electromagnetic decays of $P$ wave heavy baryons $\Lambda_{c 1}, \Lambda_{c 1}^{*}$, Phys. Rev. D 61, 114019 (2000).

[71] S. S. Agaev, K. Azizi, and H. Sundu, Decay widths of the excited $\Omega_{b}$ baryons, Phys. Rev. D 96, 094011 (2017).

[72] M. Q. Huang, Y. B. Dai, and C.S. Huang, Decays of excited charmed Lambda type and Sigma type baryons in heavy hadron chiral perturbation theory, Phys. Rev. D 52, 3986 (1995); Erratum, Phys. Rev. D55, 7317(E) (1997).

[73] G. Chiladze and A.F. Falk, Phenomenology of new baryons with charm and strangeness, Phys. Rev. D 56, R6738 (1997).

[74] P. L. Cho, Strong and electromagnetic decays of two new $\Lambda_{c}^{*}$ baryons, Phys. Rev. D 50, 3295 (1994).

[75] D. Pirjol and T. M. Yan, Predictions for $S$ wave and $P$ wave heavy baryons from sum rules and constituent quark model: Strong interactions, Phys. Rev. D 56, 5483 (1997).

[76] A. E. Blechman, A. F. Falk, D. Pirjol, and J. M. Yelton, Threshold effects in excited charmed baryon decays, Phys. Rev. D 67, 074033 (2003).

[77] S. Tawfiq, P. J. O'Donnell, and J. G. Korner, Charmed baryon strong coupling constants in a light front quark model, Phys. Rev. D 58, 054010 (1998).

[78] S. Tawfiq and P. J. O'Donnell, $P$ wave to $S$ wave pion transitions of charmed baryons, Phys. Rev. D 60, 014013 (1999).

[79] M. A. Ivanov, J. G. Korner, V. E. Lyubovitskij, and A. G. Rusetsky, Strong and radiative decays of heavy flavored baryons, Phys. Rev. D 60, 094002 (1999).

[80] M. A. Ivanov, J. G. Korner, V. E. Lyubovitskij, and A. G. Rusetsky, One pion charm baryon transitions in a relativistic three quark model, Phys. Lett. B 442, 435 (1998).

[81] F. Hussain, J. G. Korner, and S. Tawfiq, One pion transitions between heavy baryons in the constituent quark model, Phys. Rev. D 61, 114003 (2000).

[82] C. Albertus, E. Hernandez, J. Nieves, and J. M. VerdeVelasco, Study of the strong $\Sigma_{c} \rightarrow \Lambda_{c} \pi, \Sigma_{c}^{*} \rightarrow \Lambda_{c} \pi$ and $\Xi_{c}^{*} \rightarrow \Xi_{c} \pi$ decays in a nonrelativistic quark model, Phys. Rev. D 72, 094022 (2005).

[83] C. W. Hwang, Combined chiral dynamics and MIT bag model study of strong $\Sigma_{Q}^{*} \rightarrow \Lambda_{Q} \pi$ decays, Eur. Phys. J. C 50, 793 (2007).

[84] X. H. Guo, K. W. Wei, and X. H. Wu, Strong decays of heavy baryons in Bethe-Salpeter formalism, Phys. Rev. D 77, 036003 (2008). 
[85] E. Hernandez and J. Nieves, Study of the strong $\Sigma_{b} \rightarrow \Lambda_{b} \pi$ and $\Sigma_{b}^{*} \rightarrow \Lambda_{b} \pi$ in a non-relativistic quark model, Phys. Rev. D 84, 057902 (2011).

[86] A. Limphirat, C. Kobdaj, P. Suebka, and Y. Yan, Decay widths of ground-state and excited $\Xi_{b}$ baryons in a nonrelativistic quark model, Phys. Rev. C 82, 055201 (2010).

[87] T. M. Aliev, K. Azizi, and M. Savci, Strong coupling constants of light pseudoscalar mesons with heavy baryons in QCD, Phys. Lett. B 696, 220 (2011).

[88] J. G. Korner, M. Kramer, and D. Pirjol, Heavy baryons, Prog. Part. Nucl. Phys. 33, 787 (1994).

[89] H. X. Chen, W. Chen, X. Liu, Y. R. Liu, and S. L. Zhu, A review of the open charm and open bottom systems, Rep. Prog. Phys. 80, 076201 (2017).

[90] V. Crede and W. Roberts, Progress towards understanding baryon resonances, Rep. Prog. Phys. 76, 076301 (2013).

[91] E. Klempt and J. M. Richard, Baryon spectroscopy, Rev. Mod. Phys. 82, 1095 (2010).

[92] A. Manohar and H. Georgi, Chiral quarks and the nonrelativistic quark model, Nucl. Phys. B234, 189 (1984).

[93] Z. P. Li, H. X. Ye, and M. H. Lu, A unified approach to pseudoscalar meson photoproductions off nucleons in the quark model, Phys. Rev. C 56, 1099 (1997).

[94] Q. Zhao, J. S. Al-Khalili, Z. P. Li, and R. L. Workman, Pion photoproduction on the nucleon in the quark model, Phys. Rev. C 65, 065204 (2002).

[95] X.H. Zhong and Q. Zhao, Strong decays of newly observed $D_{s J}$ states in a constituent quark model with effective Lagrangians, Phys. Rev. D 81, 014031 (2010).

[96] X. H. Zhong and Q. Zhao, Strong decays of heavy-light mesons in a chiral quark model, Phys. Rev. D 78, 014029 (2008).

[97] X.H. Zhong, Strong decays of the newly observed $D(2550), D(2600), D(2750)$, and $D(2760)$, Phys. Rev. D 82, 114014 (2010).

[98] L. Y. Xiao and X. H. Zhong, Strong decays of higher excited heavy-light mesons in a chiral quark model, Phys. Rev. D 90, 074029 (2014).

[99] L. Y. Xiao, K. L. Wang, Q. F. Lü, X. H. Zhong, and S. L. Zhu, Strong and radiative decays of the doubly charmed baryons, Phys. Rev. D 96, 094005 (2017).

[100] L. Y. Xiao and X. H. Zhong, $\Xi$ baryon strong decays in a chiral quark model, Phys. Rev. D 87, 094002 (2013).

[101] L. Micu, Decay rates of meson resonances in a quark model, Nucl. Phys. B10, 521 (1969).

[102] A. Le Yaouanc, L. Oliver, O. Pene, and J. C. Raynal, Naive quark pair creation model of strong interaction vertices, Phys. Rev. D 8, 2223 (1973).

[103] A. Le Yaouanc, L. Oliver, O. Pene, and J.-C. Raynal, Naive quark pair creation model and baryon decays, Phys. Rev. D 9, 1415 (1974).

[104] W. J. Deng, H. Liu, L. C. Gui, and X. H. Zhong, Spectrum and electromagnetic transitions of bottomonium, Phys. Rev. D 95, 074002 (2017).

[105] W. J. Deng, H. Liu, L. C. Gui, and X. H. Zhong, Charmonium spectrum and their electromagnetic transitions with higher multipole contributions, Phys. Rev. D 95, 034026 (2017).

[106] Q. F. Lü, K. L. Wang, L. Y. Xiao, and X. H. Zhong, Mass spectra and radiative transitions of doubly heavy baryons in a relativized quark model, Phys. Rev. D 96, 114006 (2017).

[107] S. J. Brodsky and J. R. Primack, The electromagnetic interactions of composite systems, Ann. Phys. (N.Y.) 52, 315 (1969).

[108] Z. P. Li, The threshold pion photoproduction of nucleons in the chiral quark model, Phys. Rev. D 50, 5639 (1994).

[109] Q. Zhao, Z. p. Li, and C. Bennhold, Vector meson photoproduction with an effective Lagrangian in the quark model, Phys. Rev. C 58, 2393 (1998).

[110] L. Y. Xiao, X. Cao, and X.H. Zhong, Neutral pion photoproduction on the nucleon in a chiral quark model, Phys. Rev. C 92, 035202 (2015).

[111] X. H. Zhong and Q. Zhao, $\eta$ photoproduction on the quasifree nucleons in the chiral quark model, Phys. Rev. C 84, 045207 (2011).

[112] X. H. Zhong and Q. Zhao, $\eta^{\prime}$ photoproduction on the nucleons in the quark model, Phys. Rev. C 84, 065204 (2011).

[113] M. Artuso et al. (CLEO Collaboration), Observation of New States Decaying into $\Lambda_{c}^{+} \pi^{-} \pi^{+}$, Phys. Rev. Lett. 86, 4479 (2001).

[114] K. Abe et al. (Belle Collaboration), Experimental Constraints on the Possible $J^{P}$ Quantum Numbers of the $\Lambda_{c}(2880)^{+}$, Phys. Rev. Lett. 98, 262001 (2007).

[115] B. Aubert et al. (BABAR Collaboration), Observation of a Charmed Baryon Decaying to $D^{0} p$ at a Mass Near 2.94-GeV/c2 , Phys. Rev. Lett. 98, 012001 (2007).

[116] R. Aaij et al. (LHCb Collaboration), Observation of Overlapping Spin-1 and Spin-3 $\bar{D}^{0} K^{-}$Resonances at Mass $2.86 \mathrm{GeV} / c^{2}$, Phys. Rev. Lett. 113, 162001 (2014).

[117] P. Colangelo, F. De Fazio, and S. Nicotri, $D_{s J}(2860)$ resonance and the $s_{\ell}^{P}=\frac{5}{2}^{-} c \bar{s}(c \bar{q})$ doublet, Phys. Lett. B 642, 48 (2006).

[118] B. Zhang, X. Liu, W. Z. Deng, and S. L. Zhu, $D_{s J}(2860)$ and $D_{s J}(2715)$, Eur. Phys. J. C 50, 617 (2007).

[119] F. De Fazio, Investigating quantum numbers of new $c \bar{s}$ states, Nucl. Phys. B, Proc. Suppl. 186, 363 (2009).

[120] E. van Beveren and G. Rupp, Comment on 'Study of $D_{s J}$ decays to $D^{*} K$ in inclusive $e^{+} e^{-}$interactions,' Phys. Rev. D 81, 118101 (2010).

[121] R. Chistov et al. (Belle Collaboration), Observation of New States Decaying into $\Lambda_{c}^{+} K^{-} \pi^{+}$and $\Lambda_{c}^{+} K_{S}^{0} \pi^{-}$, Phys. Rev. Lett. 97, 162001 (2006).

[122] B. Aubert et al. (BABAR Collaboration), A study of excited charm-strange baryons with evidence for new baryons $\Xi_{c}(3055)^{+}$and $\Xi_{c}(3123)^{+}$, Phys. Rev. D 77, 012002 (2008).

[123] Y. Kato et al. (Belle Collaboration), Studies of charmed strange baryons in the $\Lambda D$ final state at Belle, Phys. Rev. D 94, 032002 (2016). 